OAK RIDGE

NATIONAL LABORATORY

MANAGED BY UT-BATTELLE

FOR THE DEPARTMENT OF ENERGY

\title{
Characterization of the Radioactive Sludge from the ORNL MVST Waste Tanks
}

\author{
J. M. Keller \\ J. M. Giaquinto
}




\section{DOCUMENT AVAILABILITY}

Reports produced after January 1, 1996, are generally available free via the U.S. Department of Energy (DOE) Information Bridge.

Web site http://www.osti.gov/bridge

Reports produced before January 1,1996 , may be purchased by members of the public from the following source.

National Technical Information Service

5285 Port Royal Road

Springfield, VA 22161

Telephone 703-605-6000 (I-800-553-6847)

TDD 703-487-4639

Fax 703-605-6900

E-mail info@ntis.fedworld.gov

Web site http://www.ntis.gov/support/ordernowabout.htm

Reports are available to DOE employees, DOE contractors, Energy Technology Data Exchange (ETDE) representatives, and International Nuclear Information System (INIS) representatives from the following source.

Office of Scientific and Technical Information

P.O. Box 62

Oak Ridge, TN 37831

Telephone 865-576-8401

Fax 865-576-5728

E-mail reports@adonis.osti.gov

Web site http://www.osti.gov/contact.htm!

This report was prepared as an account of work sponsored by an agency of the United States Government. Neither the United States Government nor any agency thereof, nor any of their employees, makes any warranty, express or implied, or assumes any legal liability or responsibility for the accuracy, completeness, or usefulness of any information, apparatus, product, or process disclosed, or represents that its use would not infringe privately owned rights. Reference herein to any specific commercial product, process, or service by trade name, trademark, manufacturer, or otherwise, does not necessarily constitute or imply its endorsement, recommendation, or favoring by the United States Government or any agency thereof. The views and opinions of authors expressed herein do not necessarily state or reflect those of the United States Government or any agency thereof. 


\title{
Characterization of the Radioactive Sludge from the ORNL MVST Waste Tanks
}

\author{
J. M. Keller \\ J. M. Giaquinto
}

September 2001

Prepared by the OAK RIDGE NATIONAL LABORATORY

Oak Ridge, Tennessee 37831 managed by UT-Battelle for the U.S. DEPARTMENT OF ENERGY under contract DE-AC05-00OR22725 


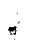




\section{TABLE OF CONTENTS}

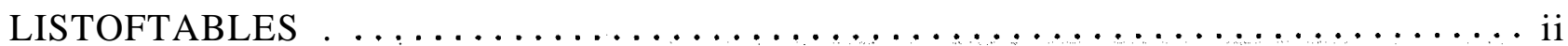

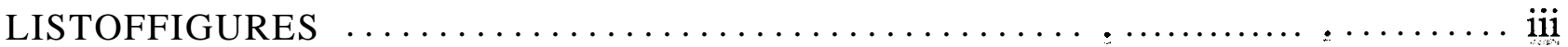

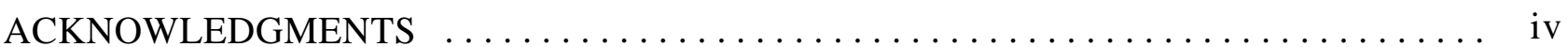

\section{ABBREVIATIONSANDACRONYMS}

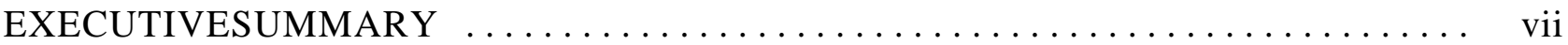

Introduction

2.0 Sample Collection Activities $\ldots \ldots \ldots \ldots \ldots \ldots \ldots \ldots \ldots \ldots \ldots \ldots \ldots \ldots \ldots \ldots$

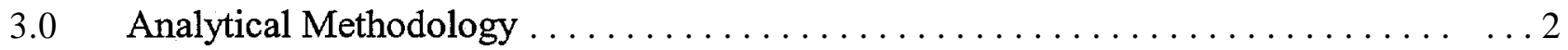

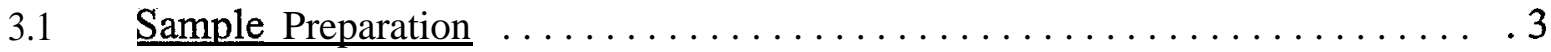

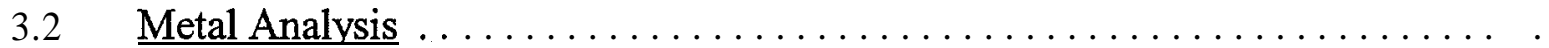

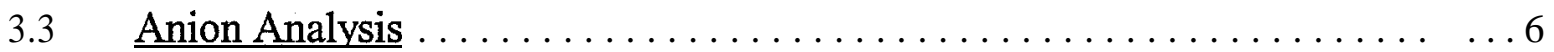

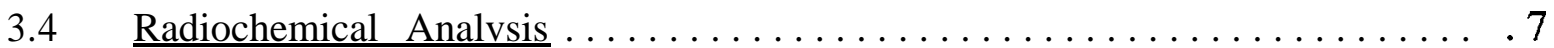

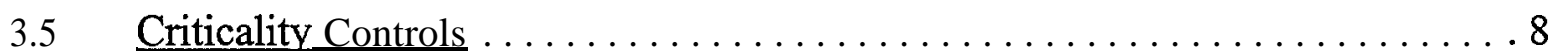

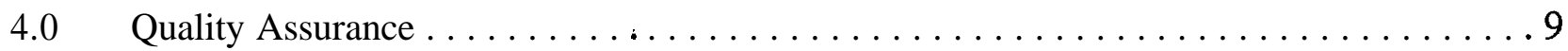

$5.0 \quad$ Summary of Inorganic and Radiochemical Analytical Results $\ldots \ldots \ldots \ldots \ldots$

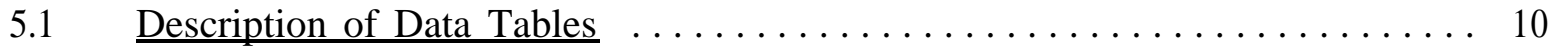

5.2 Discussion of MVST Sludge Characteristics $\ldots \ldots \ldots \ldots \ldots \ldots \ldots \ldots$

$5.4 \quad$ RCRA Characteristics for the MVST Svstem $\ldots \ldots \ldots \ldots \ldots \ldots \ldots \ldots \ldots 50$

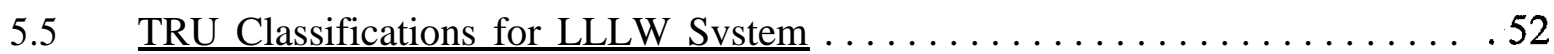

5.6 Distribution of Fissile Material in LLLW System $\ldots \ldots \ldots \ldots \ldots \ldots$

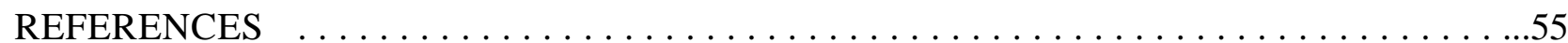

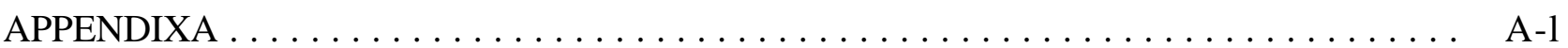

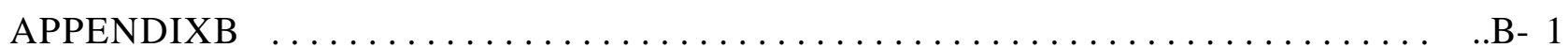

QC Acceptance Criteria for Radioactive Liquid/Solid Waste Samples ........... B-1 


\section{LIST OF TABLES}

Table 1 Summary of MVST Tanks in the ORNL LLLW System . . . . . . . . . . . . 2

Table 2 Analytical Data for Sludge in Tanks W-24 ................... 13

Table 3 Analytical Data for Sludge in Tanks W-25 ................... 17

Table 4 Analytical Data for Sludge in Tanks W-26 .................... 21

Table 5 Analytical Data for Sludge in Tanks W-27 .................. 25

Table 6 Analytical Data for Sludge in Tanks W-28 ................... 29

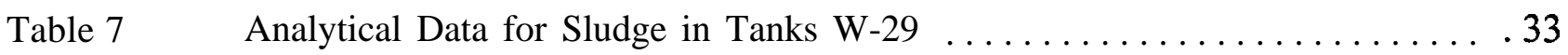

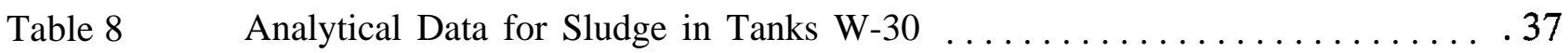

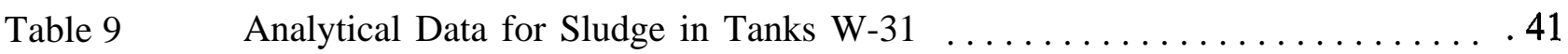

Table 10 Assumption Used for Major Compounds in MVST Sludge ............45

Table 11 Summary of Quality Checks for MVST Sludge Data ..............46

Table 12 Distribution of Beta Activity in the MVST Sludge .................. 49

Table $13 \quad$ Summary of Actinide Elements in MVST Sludge .................. . 49

Table $14 \quad$ Summary of RCRA Regulatory Limits $\ldots \ldots \ldots \ldots \ldots \ldots \ldots \ldots \ldots \ldots . .51$

Table $15 \quad$ Summary of Denature Ratios for MVST Sludge $\ldots \ldots \ldots \ldots \ldots \ldots \ldots .53$

Table 16 Example of Converting Atom $\%$ to Weight $\%$ for W-31 Sludge ........... 54 


\section{LIST OF FIGURES}

Figure 1 Distribution of Major Compounds in the MVST Sludge $\ldots \ldots \ldots \ldots 7$

Figure 2 Distribution of Uranium and Thorium in the MVST Sludge $\ldots \ldots \ldots \ldots 7$

Figure 3 Distribution of Uranium in the MVST Sludge in 1996, 2000, and $2001 \ldots 48$

Figure 4 Distribution of Plutonium Alpha Activity in the MVST Sludge ........ 54 


\section{ACKNOWLEDGMENTS}

The authors wish to express appreciation to the following staff members of the ORNL Chemical and Analytical Sciences Division who made important contributions to this work:

$\underline{\text { Inorganic and Radiochemical Analytical Support }}$

L. D. Bible

R. D. Canaan

R. Merriweather

P. D. Van Berkel

C. S. White 


\section{ABBREVIATIONS AND ACRONYMS}

\begin{tabular}{|c|c|}
\hline ALARA & As Low As Reasonably Achievable \\
\hline $\mathrm{CAO}$ & Carlsbad Area Office \\
\hline CASD & Chemical and Analytical Sciences Division \\
\hline CVAA & Cold Vapor Atomic Absorption \\
\hline DQO & Data Quality Objective \\
\hline EPA & Environmental Protection Agency \\
\hline GC/MS & Gas Chromatography/Mass Spectrometry \\
\hline $\mathrm{GC}$ & Gas Chromatography \\
\hline GFAA & Graphite Furnace Atomic Absorption \\
\hline $\mathrm{IC}$ & Ion Chromatography \\
\hline $\mathrm{ICP}$ & Inductively Coupled Plasma \\
\hline ICP-AES & Inductively Coupled Plasma - Atomic Emission Spectroscopy \\
\hline ICP-MS & Inductively Coupled Plasma - Mass Spectrometry \\
\hline IDL & Instrument Detection Limit \\
\hline LCS & Laboratory Control Sample \\
\hline LLLW & Liquid Low-Level Waste \\
\hline MDL & Method Detection Limit \\
\hline MS & Matrix Spike \\
\hline MSD & Matrix Spike Duplicate \\
\hline MVST & Melton Valley Storage Tanks \\
\hline NTS & Nevada Test Site \\
\hline ORNL & Oak Ridge National Laboratory \\
\hline QA & Quality Assurance \\
\hline QAPjP & Quality Assurance Project Plan \\
\hline QAPP & Quality Assurance Program Plan \\
\hline QC & Quality Control \\
\hline RCRA & Resource Conservation and Recovery Act \\
\hline RH-TRU & Remote Handled Transuranic Waste \\
\hline RMAL & Radioactive Materials Analytical Laboratory (Building 2026) \\
\hline $\mathrm{TC}$ & Total Carbon \\
\hline TCLP & Toxicity Characteristic Leaching Procedure \\
\hline TDS & Total Dissolved Solids \\
\hline TIC & Total Inorganic Carbon or Tentatively Identified Compounds \\
\hline TIMS & Thermal Ionization Mass Spectrometry \\
\hline TOC & Total Organic Carbon \\
\hline TRU & Transuranic \\
\hline TWCP & Transuranic Waste Characterization Program \\
\hline WAC & Waste Acceptance Criteria \\
\hline WIPP & Waste Isolation Pilot Plant \\
\hline
\end{tabular}




\section{EXECUTIVE SUMMARY}

Over the last several years most of the sludge and liquid from the Liquid Low-Level Waste (LLLW) tanks at ORNL has been transferred and consolidated in the Melton Valley Storage Tanks (MVST). The contents of the MVST tanks at the time the sludge samples were collected for this report included the original inventory in the MVSTs along with the sludge and liquid from the Bethel Valley Evaporator Service Tanks (BVEST), Old Hydrofi-acture (OHF) tanks, and the Gunite and Associated Tanks (GAAT). During the summer of2001 full core samples of sludge were collected from the MVST tanks. The purpose of this sampling campaign was to characterize and validate that the current radiochemical and chemical contents of the MVST sludge, which was needed to meet the contract agreements prior to the transfer of the waste to another DOE contractor for processing. This report only discusses the analytical characterization of the sludge from the MVST waste tanks.

The isotopic data presented in this report supports the position that fissile isotopes of uranium ${ }^{233} \mathrm{U}$ and $\left.{ }^{235} \mathrm{U}\right)$ and plutonium $\left({ }^{239} \mathrm{Pu}\right.$ and $\left.{ }^{241} \mathrm{Pu}\right)$ were "denatured" as required by the administrative controls stated in the ORNL LLLW waste acceptance criteria (WAC). In general, the MVST sludge was found to be hazardous by RCRA characteristics based on total analysis of chromium, mercury, and lead. Also, the alpha activity due to transuranic isotopes was well above the $100 \mathrm{nCi} / \mathrm{g}$ limit for TRU waste. The characteristics of the MVST sludge relative to the WIPP WAC limits for fissile gram equivalent, plutonium equivalent activity, and therrnal power from decay heat, were estimated from the data in previous reports and found to be far below the upper boundary for any of the remotehandled transuranic waste (RH-TRU) requirements for disposal of the waste in WIPP. Therefore, the WIPP WAC limits were not evaluated for this set of samples. 


\title{
Characterization of the Radioactive Sludge from the ORNL MVST Waste Tanks
}

\author{
J. M. Keller and J. M. Giaquinto
}

\subsection{Introduction}

The active ORNL Liquid Low Level Waste (LLLW) system consists of the set of waste tanks summarized in Table 1. As indicated in Table 1, this report only discusses the analytical characterization data for the Melton Valley Storage Tanks (MVST). The characterization data summarized in this report was needed to verify the current composition of the sludge present after the transfer of sludge from the Bethel Valley Evaporator Service Tanks (BVEST), Gunite and Associated Tanks (GAAT), and the Old Hydrofracture (OHF) tanks. The data in this report can also be used to address waste processing options, support the performance assessment (PA) requirements for the Waste Isolation Pilot Plant (WIPP), evaluate the waste characteristics with respect to the waste acceptance criteria (WAC) for WIPP and Nevada Test Site (NT'S), address criticality concerns, and to meet DOT requirements for transporting the waste.

The samples and data collected for this project were performed during May and June of 2001. The level of quality assurance approximates what is required for regulatory measurements with the understanding that, when needed, sample size requirements were reduced, and steps were taken to reduce sample handling to ensure radiation exposures were as-low-as-reasonably-achievable (ALARA). Some procedure modifications were required to handle chemical matrix problems due to the high levels of sodium nitrate, uranium, and thorium present. Any deviations from procedures or problems observed with the tank samples were documented in the data tiles maintained by the laboratory. The regulatory holding time requirements for mercury and the organic measurements were complied with unless noted differently in the data tables. The Quality Control (QC) Acceptance Criteria for measurements used on this project are summarized in Appendix B. 
Table $1 \quad$ Summary of MVST Tanks in the ORNL LLLW System

\begin{tabular}{||l|c|c||}
\hline \multirow{2}{*}{ Tanks } & \multicolumn{2}{|c||}{ Data Presented in this report } \\
\cline { 2 - 4 } MVST TANKS & Liquid & Sludge \\
\hline W-24 & none & $\checkmark$ \\
\hline W-25 & none & $\checkmark$ \\
\hline W-26 & none & $\checkmark$ \\
\hline W-27 & none & $\checkmark$ \\
\hline W-28 & none & $\checkmark$ \\
\hline W-29 & none & $\checkmark$ \\
\hline W-30 & none & $\checkmark$ \\
\hline w-3 1 & none & \\
\hline \hline
\end{tabular}

\subsection{Sample Collection Activities}

A detailed description on the background, operation of the LLLW system, and the sample collection techniques has been presented in previous reports and will not be discussed here (see Sections 2 and 3 of Reference 1). The staff from the Liquid and Gaseous Waste Operations (LGWO) provided all sample collection support and delivered the samples to the analytical laboratory. The tank location for sludge samples collected in 2000 are illustrated in Appendix A and there were only minor changes in the tank contents for this sampling campaign. The documentation for chain-of-custody was prepared, maintained for each sample collected, and stored with the data files by the analytical laboratory.

\subsection{Analytical Methodology}

The information and data collected from these studies are used to support various activities. The activities include demonstration of regulatory compliance, measurements to support processing options, and to meet data needs for risk assessments and other safety related assessments such as 
criticality. Standardized analytical procedures are used to the extent possible to ensure broad acceptance of the data generated. Unless stated otherwise, the U. S. Environmental Protection Agency (EPA) methods are used for. the analyses of constituents listed as, hazardous under the Resource Conservation and Recovery Act (RCRA), which includes all the inorganic and organic measurements presented in this report. In general the EPA Guidance Manual, Test Methods for Evaluating Solid Waste ${ }^{2}$ (SW-846), is used for inorganic and organic methods. Some modifications of the standard procedures are necessary to handle the high radiation levels and the high salt/solids content. Some procedure modifications are required to generate valid data, these changes were usually needed to correct for chemical or other matrix related interferences All deviations from the standard procedures are documented in the raw data files and can be provided upon request to data users.

\subsection{Sample Preparation}

The interstitial liquid samples collected from the sludge were obtained by centrifuging a well mixed suspended sludge. The clarified liquids were then digested by the SW-846 Method 3015, Microwave Assist\&d Acid Digestion of Aqueous Samples and Extracts, This sample preparation for aqueous samples was then used for all mercury analyses by CVAA.. Based upon results from a collaborative study $^{3}$ with Argonne National Laboratory - East (ANL-E), Method 3015/3051 demonstrated excellent recovery for mercury and was used to prepare tank samples for mercury determination.

The primary method for digesting the sludge samples was SW-846 Method 3051, Microwave Assisted Acid Digestion of Sediments, Sludges, Soils, and Oils. This sample preparation is considered to be a total digestion for metals and radionuclides by regulatory agencies and yields good results for most metals and radionuclides of interest. This digestion gave poor performance on two of the metals of interest, silver and silicon. Although nitric acid is excellent for dissolving' silver compounds, there is usually enough chloride present in waste samples to form an insoluble silver chloride $(\mathrm{AgCl})$ precipitate. If the chloride concentration is increased sufficiently, a silver chloride complex $\left(\mathrm{AgCl}_{3}^{-2}\right)$ forms which is soluble in the aqueous environment. Improved matrix spike recovery and defensible data for silver were obtained in earlier sampling campaigns using a separate sample digestion discussed in the earlier reports. 
If the total silicon content in the sludge must be known to develop waste treatment options such as vitrification, another sample digestion is required. A simple nitric acid treatment will not dissolve most siliceous materials. The SW-846 Method 3052, Microwave Assisted Acid Digestion of Siliceous and Organically Based Matrices, provides the necessary digestion chemistry to yield good silicon data. Sludge samples were prepared for measurement of total silicon, by taking approximately $0.5 \mathrm{~g}$ of sludge and mixing with $7 \mathrm{~mL}$ of concentrated nitric acid and $3 \mathrm{~mL}$ of hydrofluoric acid in a fluorocarbon microwave vessel. The samples were digested for 10 minutes at full power (1200 watts) holding the digestion temperature at $190^{\circ} \mathrm{C}$ and then cooled to room temperature. The acid solution was then treated with excess boric acid and heated to $80^{\circ} \mathrm{C}$ for ten minutes to complex any free fluoride. This digestion mixture is cooled, filtered into a $50 \mathrm{~mL}$ volumetric flask, and diluted to volume with ASTM Type II water. Care'must be exercised to ensure the digestion solution is cooled to room temperature prior to opening the sealed microwave vessel or there may be a significant loss of the volatile $\mathrm{SiF}_{4}$. The free fluoride is complexed with the boron to protect the sample introduction system to the ICP-AES and to prevent a high silicon background from the instrument glassware. This sample digestion with hydrofluoric acid should not be used for radiochemical measurements, especially for measurement of lanthanides or actinides.

Most of the metal and radionuclide data presented in this report are based'upon a Method 3051 digestion with approximately a 0.5 gram sludge sample and $10 \mathrm{~mL}$ of concentrated nitric acid. After the microwave digestion is completed and the solution cooled to room temperature, the sample is filtered into a volumetric flask and diluted to $50 \mathrm{~mL}$ with ASTM Type II water or better. Any residue remaining after the nitric acid digestion consisted of mostly $\mathrm{SiO}_{2}$ and was discarded. 


\subsection{Metal Analvsis}

Three analytical measurement methods were used to determine all of the metals included in this report. Most of the metals are first determined by SW-846 Method 6010A, Inductively Coupled Plasma-Atomic Emission Spectroscopy (ICP-AES). There are several elements of interest for which the ICP-AES has insufficient detection limits, and these elements must be determined by Method 7000A, Atomic Absorption Methods. The Radioactive Materials Analytical Laboratory (RMAL) uses a Graphite Furnace Atomic Absorption (GFAA) Spectrometer or Inductively Coupled Plasma-Mass Spectrometry (ICP-MS) for elements that require better sensitivity. The elements lead ( $\mathrm{Pb})$, thallium (Tl) and antimony ( $\mathrm{Sb}$ ) were analyzed by ICP-MS (Method 6020). The GFAA methods were used for arsenic (Method 7060A) and selenium (Method 7740). All the mercury measurements are done by either Method 7470A, Mercury in Liquid Waste (Manual Cold-Vapor Technique), or Method 7471A, Mercury in Solid or Semisolid Waste (Manual Cold-Vapor Technique). The samples discussed in this report were prepared for mercury analysis by the microwave technique discussed in section 3.1, the sample preparation specified in the mercury methods (7470A and $7471 \mathrm{~A})$ were not used.

The level of radioactivity in most LLLW tank samples required that the analytical systems used for metal measurements be modified for operation in a radiochemical hood or glove box. Custom instrument configurations are necessary to ensure contamination control and worker safety. All work was performed in radiochemical laboratories which are operated under strict radiation protection programs, with the use of protective clothing and routine contamination monitoring. Both an ICPAES system and a GFAA system can generate dry, dusty particles which are difficult to contain and are highly hazardous when radioactive. A detailed description of the RMAL setup for these instruments are given in Appendix B of Reference 1.

The instrument detection limits (DL) for various metals with undiluted aqueous samples are listed in data tables along with the results. For sludge samples, these detection limits must be increased by a factor that represents the dilution that results from the sample preparation. 'For all the MVST sludge samples approximately $0.5 \mathrm{~g}$ of sample was digested and then diluted to $50 \mathrm{~mL}$ which results in about a 100 fold dilution for the sample, and thus a 100 fold increase in the detection limits. 
The analytical error for the metal measurements depends upon the analytical method, the concentration level, and the chemical matrix. Inductively coupled plasma-atomic emission spectroscopy (ICP-AES) and inductively coupled plasma-mass spectrometry (ICP-MS) are both multi-element measurement techniques that are designed for the best average performance for all elements analyzed. In general, these measurement techniques are not optimized for any single element, The sample introduction system for ICP instruments adds additional variability due to changes in sample density, viscosity, and solids content between samples and/or calibration standards. Overall, the expected analytical error for ICP measurements range from $\pm 4-6 \%$ at concentrations above 10 times the detection limit to $\pm 20-50 \%$ near the detection limit. These error estimates are typical for both ICP-AES and ICP-MS measurements.

Graphite Furnace AA instruments are generally optimized for a specific element and usually provide lower detection limits and better precision. The expected analytical error for GFAA measurements range from 3-5\% for concentrations greater than 10 times the detection limit to $20-40 \%$ near the detection limit. One advantage of GFAA analysis is that the measurements are normally well above the method's detection limits. The mercury measurements were done by Cold Vapor Atomic Absorption (CVAA), which is very selective and sensitive for mercury. The analytical errors for CVAA measurements are similar to GFAA work.

\subsection{Anion Analvsis}

The determination of the inorganic anions was needed for the development of process treatment options, to provide information to explain the distribution and chemical behaviors observed in the waste tanks, and to ensure the major chemical constituents were identified in the waste for which data was used to calculate the mass and charge balance for each sample. The common inorganic anions; including fluoride, chloride, bromide, phosphate, nitrate, nitrite, and sulfate; were measured by ion chromatography (IC) with a Dionex Model DX600 system. In addition, several water soluble organic acids were measured along with the inorganic anions. These organic acids were measured in their ionized form and included formate, acetate, citrate, and oxalate. Both the citrate and the oxalate can form strong complexes with many metals and change the solution chemistry of these metals in the waste. The ion chromatography system used for measurements on these radioactive 
samples was configured such that the components that come into contact with radioactivity were isolated in a radiochemical hood for contamination control,

From past observations, the nitrate content dominates both the mass and charge balance calculations with sludge samples taken from the active LLLW tanks. There are many other anions present in the waste, some of which are measured directly by ion chromatography and others which can be estimated from the metal data such as chromate, dichromate, permanganate, and others. The carbonate is estimated from the total inorganic carbon measurement.

The anion measurement technique was ion chromatography. For simple water samples, without complex chemical matrix problems, the empirical analytical error for ion chromatography measurements ranges from $4-6 \%$ for concentrations above 10 times the detection limits to $20-40 \%$ near the detection limit. The measurement of anions present at concentration much lower $(<1 / 25)$ than other anionic species present may increase the overall error of the measurement.

\subsection{Radiochemical Analysis}

The only standard radiochemical methods useful for radioactive waste characterization are EPA Method 600/900.0, Gross Alpha and Beta Radioactivity in Drinking Water, and EPA Method 600/901.1: Gamma Emitting Radionuclides in Drinking Water. The EPA Method 6001905.0, Radioactive Strontium in Drinking Water, gave poor performance with the chemical matrix found in ORNL LLLW supematant and sludge samples. The EPA method for gross alpha/beta measurements uses gas-flow proportional counting. In general, this counting technique requires drying a sample at elevated temperatures onto a metal (usually stainless steel) plate, which resulted in the loss of cesium chloride from the MVST samples and yielded poor gross beta results. To avoid this problem, all gross beta measurements reported are based on measurements by liquid scintillation counting. Other than the gamma spectroscopy measurements, all of the radionuclide measurements were done with in-house procedures. The method detection limits for radiochemical measurements are dependent on both sample matrix and count time and are not listed here. In general, the radiochemical measurements used count times to yield at least $1 \%(10,000$ counts) counting statistics. The expected errors for the radiochemical data range from $\pm 5-10 \%$ for gross alpha/beta 
and gamma emitter measurements to \pm 1 O-20 \% for radionuclides that require chemical separations before counting (i.e. ${ }^{99} \mathrm{Tc},{ }^{90} \mathrm{Sr},{ }^{129} \mathrm{I}$, and ${ }^{237} \mathrm{~Np}$ ).

\subsection{Criticality Controls}

The current ORNL waste acceptance criteria (WAC) for liquid-low level waste requires that the fissile isotopes of uranium and plutonium be isotopically diluted with ${ }^{238} \mathrm{U}$ and ${ }^{232} \mathrm{Th}$, respectively. These administrative controls require that the ratio of the ${ }^{238} \mathrm{U}$ mass divided by the fissile equivalent mass (FEM) for uranium be greater than 100. The ${ }^{235} \mathrm{U}$ FEM is a useful scale for criticality calculations that normalizes the fission probability for each fissile isotope to ${ }^{235} \mathrm{U}$. These FEM factors, designated as $f_{35}$ for ${ }^{235} \mathrm{U}$ mass factors, are discussed and listed in the Appendix A, Table 1 of the ORNL Procedure NCS-1 .0, Nuclear Criticality Safety Program.

The major fissile isotopes of concern in the ORNL waste tanks are ${ }^{233} \mathrm{U},{ }^{235} \mathrm{U}$, and ${ }^{239} \mathrm{Pu}$. The fissile isotope ${ }^{241} \mathrm{Pu}$ is also present in the waste but the mass is usually several orders of magnitude lower and below a level that would influence the isotopic dilution ratio for plutonium. Other fissile isotopes present in the ORNL waste include isotopes of neptunium, americium, and curium, but the actual mass present in the waste has been too low for major concern, and the low concentration would make it difficult and expensive to measure by mass spectrometry.

The data presented in this report for isotopic dilution ratios (also referred to as denature ratios) reflect both the current ORNL standard practices for disposal of fissile isotopes of uranium and plutonium. The administrative controls which were in effect when the waste was generated were different than current practices.

All calculations dealing with isotopic dilution for criticality safety are based on isotope mass ratios and must not be confused with activity ratios. For any data discussed in this report that uses ${ }^{232} \mathrm{Th}$ relative to isotopic mass ratios, the total thorium concentration and the ${ }^{232} \mathrm{Th}$ concentration are the same value. 
The current administrative requirements for criticality control are more conservative than past practices and require that the following conditions be satisfied for uranium,

$$
\begin{aligned}
& \frac{\left({ }^{238} U\right)-200\left({ }^{233} U\right)}{\left({ }^{235} U\right)} \geq 110 \\
& \frac{\left({ }^{238} U\right)-110\left({ }^{235} U\right)}{\left({ }^{233} U\right)} \geq 200
\end{aligned}
$$

The administrative controls for plutonium require a dilution ratio of 200 parts thorium to one part plutonium (past practices only required a ratio of 100).

\subsection{Quality Assurance}

Quality assurance during the sampling activities was primarily addressed by the use of approved procedures for sampling the sludge phase found in each waste tank. These procedures provide detailed instructions for the collection, labeling, and shipping of each sample. Chain-of-custody forms were used to track individual samples from their collection point to the analytical laboratory.

The QA for the sludge characterization was based upon the RMAL Radioactive 'Waste Characterization QA Plan ${ }^{4}$ which defines the basis for quality assurance and quality control used for the analysis of the waste tank samples. The QA plans discuss staff qualification requirements, laboratory participation in performance demonstration programs, quality control acceptance criteria for analytical methods, sample management, and most other laboratory operations. The QA plans implemented at the RMAL for waste characterization meet both the WIPP and the Nevada Test Site (NTS) QA requirements for inorganic, organic, and radiochemical measurements. 


\subsection{Summary of Inorganic and Radiochemical Analytical Results}

\subsection{Description of Data Tables}

A summary of the inorganic, physical, and radiochemical analytical results for MVST sludge samples are presented in Table 2 through Table 7. Also, MVST data ${ }^{5}$ collected in 1996 was included in these data tables for comparison. These tables are arranged in a similar format to facilitate comparing data from different tanks and to group information into useful units. The analytical data presented in these tables are the consolidation of data from a single project which had a fixed set of analytical requirements. Any parameter reported with a dash ("“") indicates that the data was not measured for that sample.

The first section, "Physical properties and miscellaneous data", includes unrelated information that does not fit well into other table groups. The first parameters entered in a column include the RMAL request and sample numbers, which are laboratory filing codes used to track sample information. The next set of data includes information on the moisture or water content and the solids content of the sample. The group is completed with data on the inorganic and organic carbon content. For MVST waste tank samples the inorganic carbon can be assumed to be all carbonate and bicarbonate. The Total Organic Carbon (TOC) provides an upper limit on the organic content in the tank waste but does not include volatile organic compounds. Most of the liquid waste in the active system has been through an evaporator which removes the highly volatile organic compounds from the waste.

The next two sections include groups of metals; the "RCRA metals" are separated out for quick reference. The regulatory limit for the concentrations are listed in parentheses next to each RCRA metal. For the liquid samples the RCRA regulatory limits are used directly, since the supernatant would be defined as the TCLP leachate in the determination of waste characteristics for hazardous waste. The RCRA metal sludge data represents total metal measurements, as defined by EPA. Exceeding the RCRA regulatory limits listed for the sludge samples only indicates that the waste has the potential to be classified as hazardous. The sludge waste should only be classified as RCRA waste if the final waste form fails the TCLP leaching test. 
The remaining metals are grouped under "Process metals", which includes the common Group IA \& IIA metals along with elements that could effect chemical processing, criticality concerns, and stabilization techniques such as grouting or vitrification. For the sludge data, all the metals are reported on a "as received" (wet weight) basis.

The section "Semi-quantitative metals by ICP-MS" includes additional metals identified in a full mass range scan by inductively-coupled plasma - mass spectrometry. This measurement helps ensure all major elements have been identified in the waste. Each element reported is not calibrated but is based upon a response factor from a curve generated from a few elements across the mass range. Therefore, these elemental concentrations are listed as estimates only.

The "Anions by ion chromatography" section includes the inorganic anions, several common water soluble organic acids. Two of the organic acids included, citrate and oxalate, are also classified as complexing agents.

The "Beta/gamma emitters" section summarizes the radionuclides that emit gamma-rays and beta particles. This section includes the gross beta activity, radionuclides identified by gamma spectrometry, and several "pure" beta emitters of interest. Many of the "pure" beta emitters $\left({ }^{3} \mathrm{H},{ }^{14} \mathrm{C}\right.$, and ${ }^{90} \mathrm{Sr}$ ) require radiochemical separations prior to measurement by either liquid scintillation or gasflow proportional counting. The ${ }^{99} \mathrm{Tc}$ was measured by ICP-MS without any prior chemical separation.

The "Alpha emitters" section summarizes the actinide elements in the waste. This section includes the gross alpha activity, an estimate of the activity for each alpha emitter identified in a gross alpha spectrum, and plutonium isotopes determined by alpha spectrometry after a radiochemical separation. For supernatant samples, an estimate of the ${ }^{232} \mathrm{Th}^{239} \mathrm{Pu}$ mass ratio is included in this section to address criticality concerns if enough thorium is present to calculate the ratio. For the sludge samples, this mass ratio is included with the plutonium mass spectrometry data. 
The remaining sections include "Uranium isotopes by TIMS" " “Plutonium isotopes by $\underline{\text { TIMS", and }}$ "Uranium isotopes bv ICP-MS". These sections summarize the uranium and plutonium data measured by thermal ionization mass spectrometry and, for more recent measurements, the uranium isotopes by ICP-MS. Also, included in these sections are the isotopic mass dilution or "denature" ratios for uranium and plutonium based on the requirements in place when the waste was generated (see section 3.5). The plutonium section for the sludge samples also includes the activity for each plutonium isotope, which was calculated from the mass spectrometry data. 
Tiable 2' Analytical Data for Sludge in Tanks W-24

\begin{tabular}{|c|c|c|c|c|c|c|}
\hline \multicolumn{3}{|c|}{$\begin{array}{l}\text { Characteristic } \\
\text { (Analysis) }\end{array}$} & $\begin{array}{r}1996 \\
\mathrm{w}-24 \mathrm{~s} \\
\end{array}$ & $\begin{array}{c}2000 \\
\mathrm{w}-24 \mathrm{~s} \\
\end{array}$ & $\begin{array}{c}2001 \\
\mathrm{~W}-24 \mathrm{~S} \\
\end{array}$ & IDL' \\
\hline \multicolumn{7}{|c|}{ IPhysical properties and miscellaneous data } \\
\hline \multicolumn{3}{|c|}{$\begin{array}{l}\text { Request number } \\
\text { Sample number } \\
\text { pH }\end{array}$} & $\begin{array}{c}\mathbf{7 7 4 9 c} \\
\mathbf{9 6 0 8 0 6 - 0 0 6} \\
12.8\end{array}$ & $\begin{array}{r}10224 . \\
000509-001 \\
9.8\end{array}$ & $\begin{array}{r}12057 \\
010716-010 \\
9.96\end{array}$ & \\
\hline \multirow{7}{*}{\multicolumn{2}{|c|}{$\begin{array}{l}\text { Water }^{2} \\
\text { TS }^{\mathrm{b}} \\
\text { TSS }^{\mathrm{c}} \\
\text { TDS }^{\mathrm{d}} \\
\text { JBulk density } \\
\text { TC }^{\mathrm{e}} \\
\text { TIC }^{\mathrm{f}} \\
\text { TOC }^{\mathrm{g}}\end{array}$}} & $(\%)$ & 51.2 & 64.0 & 57.6 & \\
\hline & & $(\mathrm{mg} / \mathrm{g})$ & 488 & 360 & 424 & \\
\hline & & $(\mathrm{mg} / \mathrm{g})$ & & 253 & - & \\
\hline & & $(\mathrm{mg} / \mathrm{g})$ & & 107 & & \\
\hline & & $(\mathrm{g} / \mathrm{mL})$ & 1.37 & 1.315 & 1.276 & - \\
\hline & & $\begin{array}{l}(\mathrm{mg} / \mathrm{Kg}) \\
(\mathrm{mg} / \mathrm{Kg})\end{array}$ & $\begin{array}{l}13700 \\
13700\end{array}$ & $\begin{array}{r}28800 \\
8790\end{array}$ & $\begin{array}{r}22000 \\
8500\end{array}$ & $\begin{array}{l}15 \\
15\end{array}$ \\
\hline & & $(\mathrm{mg} / \mathrm{Kg})$ & $<15$ & 20000 & 13400 & 15 \\
\hline \multicolumn{7}{|c|}{ RCRA Metals $( \pm 10 \%)$} \\
\hline $\mathrm{Ag}^{\mathrm{h}}$ & $(100)^{\prime}$ & $(\mathrm{mg} / \mathrm{Kg})$ & $<1.9$ & & & 0.012 \\
\hline & $(100)$ & $(\mathrm{mg} / \mathrm{Kg})$ & $<5.3$ & $<0.8$ & $<1.7$ & 0.005 \\
\hline & $(2000)$ & $(\mathrm{mg} / \mathrm{Kg})$ & 75.5 & 86.2 & 107 & 0.001 \\
\hline & $(20)$ & $(\mathrm{mg} / \mathrm{Kg})$ & 13.9 & 21.1 & 37.1 & 0.168 \\
\hline & $(100)$ & $(\mathrm{mg} / \mathrm{Kg})$ & 61.6 & 236 & 276 & 0.013 \\
\hline $\mathrm{Hg}$ & (4) & $(\mathrm{mg} / \mathrm{Kg})$ & 38.0 & 74.0 & 49.9 & 0.002 \\
\hline & (1000) & $(\mathrm{mg} / \mathrm{Kg})$ & 45.2 & 76.3 & 90.0 & 0.078 \\
\hline $\mathrm{Pb}$ & $(100)$ & $(\mathrm{mg} / \mathrm{Kg})$ & 303 & 435 & 606 & 0.341 \\
\hline & $(20)$ & $(\mathrm{mg} / \mathrm{Kg})$ & $<5.3$ & $<0.8$ & $<4$ & 0.005 \\
\hline & $(18)$ & $(\mathrm{mg} / \mathrm{Kg})$ & $<5.3$ & $<0.8$ & $<4$ & 0.005 \\
\hline \multicolumn{7}{|c|}{ Process metals $( \pm 10 \%)$} \\
\hline & $(\mathrm{mg} / \mathrm{Kg})$ & 3330 & 3540 & 4130 & 0.035 \\
\hline \multicolumn{2}{|l|}{$\mathrm{B}$} & $(\mathrm{mg} / \mathrm{Kg})$ & 4.35 & 9.52 & 5.72 & 0.01 \\
\hline \multicolumn{2}{|l|}{$\mathrm{Be}$} & $(\mathrm{mg} / \mathrm{Kg})$ & 4.45 & 7.28 & 6.42 & 0.001 \\
\hline \multicolumn{2}{|l|}{$\mathrm{Ca}$} & $(\mathrm{mg} / \mathrm{Kg})$ & 51200 & 42400 & 55400 & 0.03 \\
\hline \multirow{2}{*}{\multicolumn{2}{|c|}{ co }} & $(\mathrm{mg} / \mathrm{Kg})$ & 2.42 & 22.9 & 42.3 & 0.039 \\
\hline & & $(\mathrm{mg} / \mathrm{Kg})$ & 28.5 & 67.4 & 70.6 & 0.006 \\
\hline \multicolumn{2}{|l|}{$\mathrm{Cd}$} & $(\mathrm{mg} / \mathrm{Kg})$ & 0.900 & 0.409 & $<4$ & 0.005 \\
\hline \multicolumn{2}{|l|}{$\begin{array}{l}\mathrm{Fe} \\
\mathrm{K}\end{array}$} & $(\mathrm{mg} / \mathrm{Kg})$ & 1250 & 2990 & 3010 & 0.014 \\
\hline \multirow{2}{*}{\multicolumn{2}{|c|}{$\begin{array}{l}\mathrm{K} \\
\mathrm{Mg}\end{array}$}} & $(\mathrm{mg} / \mathrm{Kg})$ & 13400 & 7980 & $\begin{array}{llll}9 & 6 & 0 & 0\end{array}$ & 0.5 \\
\hline & & $(\mathrm{mg} / \mathrm{Kg})$ & 9280 & 6330 & 9270 & 0.049 \\
\hline \multicolumn{2}{|l|}{$\begin{array}{l}\mathrm{Mn} \\
\mathrm{Mo}\end{array}$} & $(\mathrm{mg} / \mathrm{Kg})$ & 84.7 & 911 & 761 & 0.002 \\
\hline \multirow{2}{*}{\multicolumn{2}{|c|}{$\begin{array}{l}\mathrm{Mo} \\
\mathrm{Na}\end{array}$}} & $(\mathrm{mg} / \mathrm{Kg})$ & & 15.1 & 56.8 & 0.038 \\
\hline \multirow{2}{*}{\multicolumn{2}{|c|}{$\begin{array}{l}\mathrm{Na} \\
\mathrm{P}\end{array}$}} & $(\mathrm{mg} / \mathrm{Kg})$ & 48800 & 35200 & 40000 & 0.075 \\
\hline & & $(\mathrm{mg} / \mathrm{Kg})$ & 1240 & & - & 0.13 \\
\hline & $(\mathrm{mg} / \mathrm{Kg})$ & $<19$ & $<41$ & $<4$ & 0.509 \\
\hline \multicolumn{2}{|l|}{$\mathrm{Si}^{\mathrm{k}}$} & $(\mathrm{mg} / \mathrm{Kg})$ & 3820 & 2340 & 3490 & 0.022 \\
\hline \multicolumn{2}{|l|}{$\mathrm{Sr}$} & $(\mathrm{mg} / \mathrm{Kg})$ & 283 & 208 & 247 & 0.001 \\
\hline \multirow{2}{*}{\multicolumn{2}{|c|}{$\begin{array}{l}\mathrm{Th} \\
\mathrm{Ti}\end{array}$}} & $(\mathrm{mg} / \mathrm{Kg})$ & 3270 & 7480 & 7290 & 0.376 \\
\hline & & $(\mathrm{mg} / \mathrm{Kg})$ & & & 30.0 & 0.010 \\
\hline \multicolumn{2}{|l|}{$\mathrm{U}$} & $(\mathrm{mg} / \mathrm{Kg})$ & 6780 & 46500 & 33800 & 0.077 \\
\hline \multicolumn{2}{|l|}{$\mathrm{V}$} & $(\mathrm{mg} / \mathrm{Kg})$ & 2.23 & $<1$ & $<1$ & 0.013 \\
\hline & & $(\mathrm{mg} / \mathrm{Kg})$ & 479 & 658 & 700 & 0.445 \\
\hline $\mathrm{Zr}$ & & $(\mathrm{mg} / \mathrm{Kg})$ & & & 244 & 0.008 \\
\hline
\end{tabular}




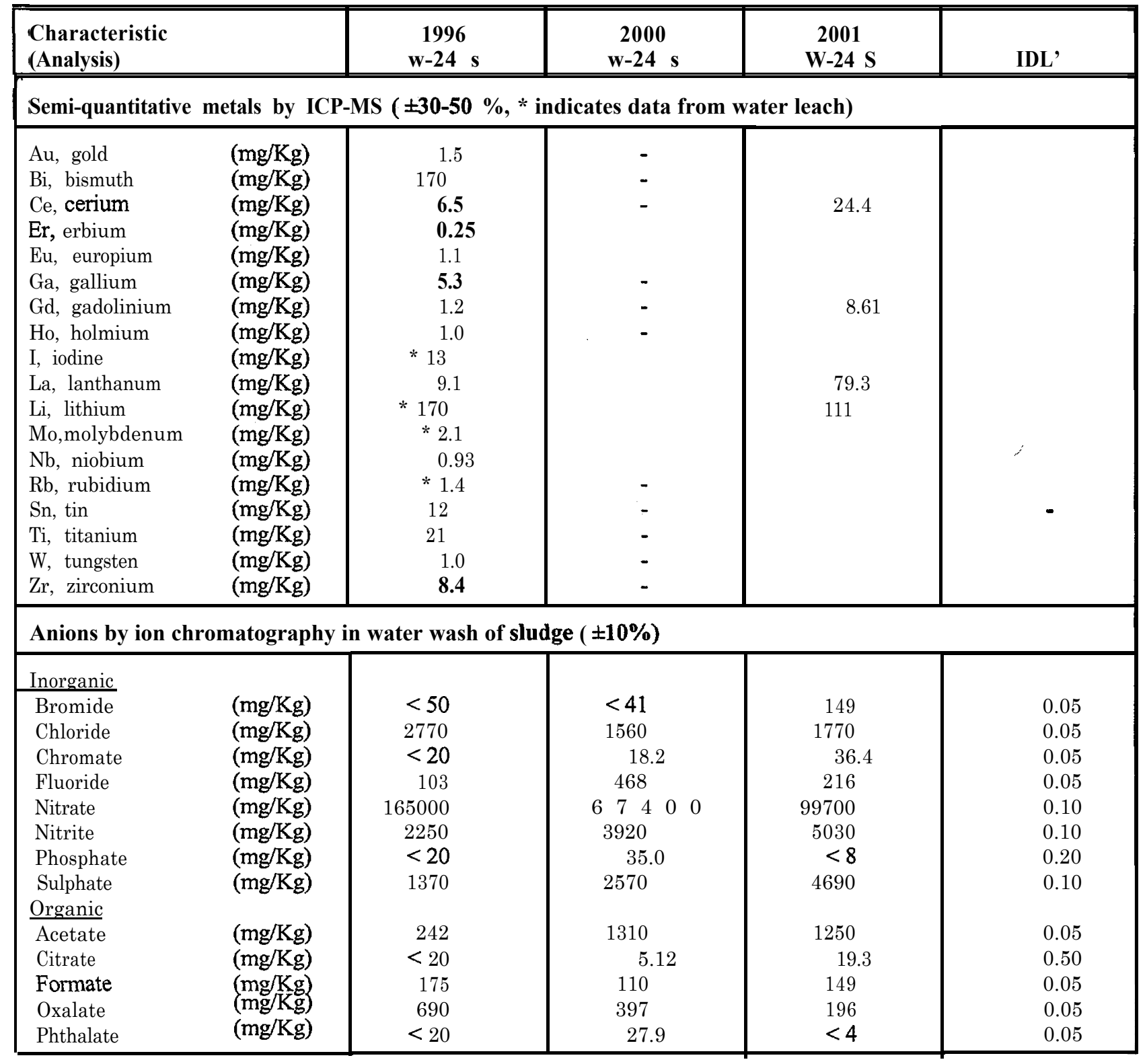




\begin{tabular}{|c|c|c|c|c|c|}
\hline $\begin{array}{l}\text { Tharacteristic } \\
\text { Analysis) }\end{array}$ & & $\begin{array}{c}1996 \\
\mathrm{~W}-24 \mathrm{~S} \\
\end{array}$ & $\begin{array}{c}2000 \\
\mathbf{W}-24 \mathbf{S} \\
\end{array}$ & $\begin{array}{c}2001 \\
\mathbf{W}-24 \mathbf{S} \\
\end{array}$ & IDL' \\
\hline \multicolumn{6}{|c|}{ Beta/gamma emitters $( \pm 10 \%)$} \\
\hline $\begin{array}{l}\text { Tross beta } \\
{ }^{i 9} \mathrm{Ni} \\
{ }^{33} \mathrm{Ni} \\
{ }^{80} \mathrm{Co} \\
{ }^{20} \mathrm{Sr} /{ }^{90} \mathrm{Y} \\
{ }^{19} \mathrm{Tc} \\
{ }^{1291} \\
{ }^{134} \mathrm{Cs} \\
{ }^{137} \mathrm{Cs} \\
{ }^{151} \mathrm{Sm} \\
{ }^{155} \mathrm{Eu} \\
{ }^{154} \mathrm{Eu} \\
{ }^{155} \mathrm{Eu} \\
{ }^{227} \mathrm{Ac} \\
{ }^{241} \mathrm{Pu}\end{array}$ & $\begin{array}{l}(\mathrm{Bq} / \mathrm{g}) \\
(\mathrm{Bq} / \mathrm{g}) \\
(\mathrm{Bq} / \mathrm{g}) \\
(\mathrm{Bq} / \mathrm{g}) \\
(\mathrm{Bq} / \mathrm{g}) \\
(\mathrm{Bq} / \mathrm{g}) \\
(\mathrm{Bq} / \mathrm{g}) \\
(\mathrm{Bq} / \mathrm{g}) \\
(\mathrm{Bq} / \mathrm{g}) \\
(\mathrm{Bq} / \mathrm{g}) \\
(\mathrm{Bq} / \mathrm{g}) \\
(\mathrm{Bq} / \mathrm{g}) \\
(\mathrm{Bq} / \mathrm{g}) \\
(\mathrm{Bq} / \mathrm{g}) \\
(\mathrm{Bq} / \mathrm{g})\end{array}$ & $\begin{array}{r}4.6 \mathrm{e}+06 \\
<2.5 \mathrm{e}+01 \\
3.3 \mathrm{e}+03 \\
2.8 \mathrm{e}+04 \\
1.4 \mathrm{e}+06 \\
4.5 \mathrm{e}+02 \\
\\
1.3 \mathrm{e}+04 \\
5.3 \mathrm{e}+05 \\
<6.0 \mathrm{e}+02 \\
8.9 \mathrm{e}+04 \\
3.8 \mathrm{e}+04 \\
1.0 \mathrm{e}+04 \\
<4.7 \mathrm{e}+03 \\
1.4 \mathrm{e}+04\end{array}$ & $\begin{array}{l}4.9 e+06 \\
3.0 e+04 \\
1.3 e+06 \\
- \\
6.3 e+05 \\
2.4 e+05 \\
2.5 e+05 \\
5.1 e+04\end{array}$ & $\begin{array}{l}4.4 \mathrm{e}+06 \\
2.5 \mathrm{e}+04 \\
1.2 \mathrm{e}+06 \\
7.9 \mathrm{e}+02 \\
- \\
3.3 \mathrm{e}+03 \\
5.5 \mathrm{e}+05 \\
6.9 \mathrm{e}+05 \\
2.2 \mathrm{e}+05 \\
6.6 \mathrm{e}+04\end{array}$ & $\begin{array}{l}- \\
- \\
-\end{array}$ \\
\hline \multicolumn{6}{|c|}{ Alpha emitters $( \pm \mathbf{1 0} \%)$} \\
\hline $\begin{array}{l}\text { Gross alpha } \\
{ }^{232} \mathrm{Th} \\
{ }^{233} \mathrm{U} \\
{ }^{234} \mathrm{U} \\
{ }^{235 \mathrm{U}} \\
{ }^{233} \mathrm{U} \\
{ }^{237} \mathrm{~Np} \\
{ }^{241} \mathrm{Am} \\
{ }^{244} \mathrm{Cm} \\
{ }^{250} \mathrm{Cf} \\
{ }^{252} \mathrm{Cf} \\
\frac{\text { Total Pu alpha }}{{ }^{238} \mathrm{Pu}} \\
{ }^{239} \mathrm{Pu} /{ }^{240} \mathrm{Pu} \\
{ }^{242} \mathrm{Pu} \\
\text { TRU activity } \\
\mathrm{Pu}+\mathrm{Am} \mathrm{(3700)}\end{array}$ & $\begin{array}{l}(\mathrm{Bq} / \mathrm{g}) \\
(\mathrm{Bq} / \mathrm{g}) \\
(\mathrm{Bq} / \mathrm{g}) \\
(\mathrm{Bq} / \mathrm{g}) \\
(\mathrm{Bq} / \mathrm{g}) \\
(\mathrm{Bq} / \mathrm{g}) \\
(\mathrm{Bq} / \mathrm{g}) \\
(\mathrm{Bq} / \mathrm{g}) \\
(\mathrm{Bq} / \mathrm{g}) \\
(\mathrm{Bq} / \mathrm{g}) \\
(\mathrm{Bq} / \mathrm{g}) \\
(\mathrm{Bq} / \mathrm{g}) \\
(\mathrm{Bq} / \mathrm{g}) \\
(\mathrm{Bq} / \mathrm{g}) \\
(\mathrm{Bq} / \mathrm{g})\end{array}$ & $\begin{array}{c}34000 \\
13 \\
1600 \\
77 \\
2.6 \\
84 \\
10 \\
3900 \\
22000 \\
<100 \\
<100 \\
\\
6600 \\
4000 \\
2600\end{array}$ & $\begin{array}{r}200000 \\
30 \\
8900 \\
710 \\
15 \\
580 \\
\\
10400 \\
155000\end{array}$ & $\begin{array}{c}150000 \\
30 \\
640 \\
184 \\
9.9 \\
419 \\
15000 \\
105000 \\
-\end{array}$ & \\
\hline \multicolumn{6}{|c|}{ Uranium isotopics by ICP-MS ( $\pm 2 \%)$} \\
\hline $\begin{array}{l}{ }^{233} \mathrm{U} \\
{ }^{234} \mathrm{U} \\
{ }_{235} \mathrm{U} \\
{ }^{236} \mathrm{U} \\
{ }^{238} \mathrm{U} \\
\\
{ }^{233} \mathrm{U} / \mathrm{MS} \\
{ }^{235} \mathrm{U} / \mathrm{MS} \\
{ }^{238} \mathrm{U} /{ }^{235} \mathrm{U} \text { FEM }\end{array}$ & $\begin{array}{l}(\text { atom \%) } \\
(\text { atom \%) } \\
(\text { atom \%) } \\
(\text { atom \%) } \\
(\text { atom \%) } \\
(\mathrm{mg} / \mathrm{Kg}) \\
(\mathrm{mg} / \mathrm{Kg}) \\
\mathrm{m} \\
-\end{array}$ & $\begin{array}{c}0.067 \\
0.005 \\
0.543 \\
0.006 \\
99.379 \\
\\
4.45 \\
36.4 \\
159\end{array}$ & $\begin{array}{l}0.0548 \\
0.0067 \\
0.4606 \\
0.0028 \\
99.475 \quad 1 \\
\\
24.9 \\
211 \\
189\end{array}$ & $\begin{array}{l}0.0546 \\
0.0024 \\
0.4157 \\
0.0032 \\
99.5241 \\
\\
18.1 \\
139 \\
206\end{array}$ & $\begin{array}{l}0.001 \\
0.001 \\
0.001 \\
0.001 \\
0.001\end{array}$ \\
\hline
\end{tabular}




\begin{tabular}{|c|c|c|c|c|c|}
\hline $\begin{array}{l}\text { Characteristic } \\
\text { (Analysis) }\end{array}$ & & $\begin{array}{c}1996 \\
\mathrm{~W}-24 \mathrm{~S} \\
\end{array}$ & $\begin{array}{c}2000 \\
\text { W-24 S } \\
\end{array}$ & $\begin{array}{c}2001 \\
\mathrm{~W}-24 \mathrm{~S} \\
\end{array}$ & IDL' \\
\hline \multicolumn{6}{|c|}{ Plutonium isotopics by TIMS $( \pm \mathbf{1 \%})$} \\
\hline $\begin{array}{l}{ }^{238} \mathrm{Pu} \\
{ }^{239} \mathrm{Pu} \\
{ }^{240} \mathrm{Pu} \\
{ }^{2 “} \mathrm{Pu} \\
{ }^{242} \mathrm{Pu} \\
{ }^{244} \mathrm{Pu}\end{array}$ & $\begin{array}{l}(\text { atom \%) } \\
(\text { atom \%) } \\
(\text { atom \%) } \\
(\text { atom \%) } \\
(\text { atom \%) } \\
(\text { atom \%) }\end{array}$ & $\begin{array}{r}\mathbf{0 . 6 3} \\
87.14 \\
10.81 \\
\mathbf{0 . 3 7} \\
1.05 \\
<0.01\end{array}$ & - & & \\
\hline $\begin{array}{l}\frac{\mathrm{Pu} \text { activity }}{{ }^{238} \mathrm{Pu}} \\
{ }^{239} \mathrm{Pu} \\
{ }^{240} \mathrm{Pu} \\
{ }^{241} \mathrm{Pu} \\
{ }^{242} \mathrm{Pu} \\
{ }^{244} \mathrm{Pu} \\
\left({ }^{239} \mathrm{Pu}\right) \\
{ }^{232} \mathrm{Th}{ }^{239} \mathrm{Pu}^{\mathrm{m}}\end{array}$ & $\begin{array}{l}(\mathrm{Bq} / \mathrm{g}) \\
(\mathrm{Bq} / \mathrm{g}) \\
(\mathrm{Bq} / \mathrm{g}) \\
(\mathrm{Bq} / \mathrm{g}) \\
(\mathrm{Bq} / \mathrm{g}) \\
(\mathrm{Bq} / \mathrm{g}) \\
(\mathrm{ng} / \mathrm{g})\end{array}$ & $\begin{array}{c}\mathbf{3 8 0 0} \\
1900 \\
\mathbf{8 7 0} \\
14000 \\
1.5 \\
<0.1 \\
\\
960 \\
3920\end{array}$ & 613 & 761 & \\
\hline \multicolumn{6}{|c|}{$\begin{array}{l}\text { (a) Free water content of sludge, (b) Total solids, (c) Total suspended solids, (d) Total dissolved solids, (e) Total } \\
\text { carbon, (f) Total inorganic carbon, (g) Total organic carbon, (h) nitric-hydrochloric acid prep., (i) RCRA regulatory } \\
\text { limits, (j) measured by ICP-MS or GHAA, (k) nitric-hydrofluoric acid prep., (l) Instrument detection limits, and (m) } \\
\text { the ratio reported for year } 2000 \text { assumes all alpha activity is from }{ }^{239} \text { Pu. }\end{array}$} \\
\hline
\end{tabular}


Table 3 Analytical Data for Sludge in Tanks W-25

\begin{tabular}{|c|c|c|c|c|c|}
\hline \multicolumn{2}{|l|}{$\begin{array}{l}\text { Characteristic } \\
\text { (Analysis) }\end{array}$} & $\begin{array}{c}1996 \\
\text { W-25 S } \\
\end{array}$ & $\begin{array}{c}2000 \\
\mathbf{W}-25 \mathrm{~S} \\
\end{array}$ & $\begin{array}{c}2001 \\
\mathbf{W}-25 \mathrm{~S} \\
\end{array}$ & IDL' \\
\hline \multicolumn{6}{|c|}{ IPhysical properties and miscellaneous data } \\
\hline $\begin{array}{l}\text { Request number } \\
\text { Sample number } \\
\text { pH } \\
\text { Water }^{\mathbf{2}} \\
\text { TS }^{\text {b }} \\
\text { TSS }^{\mathbf{c}} \\
\text { TDS }^{d} \\
\text { Bulk density }_{\text {TC }} \\
\text { TIC' }^{\text {TOC }}\end{array}$ & $\begin{array}{l}(\%) \\
(\mathrm{mg} / \mathrm{g}) \\
(\mathrm{mg} / \mathrm{g}) \\
(\mathrm{mg} / \mathrm{g}) \\
(\mathrm{g} / \mathrm{mL}) \\
(\mathrm{mg} / \mathrm{Kg}) \\
(\mathrm{mg} / \mathrm{Kg}) \\
(\mathrm{mg} / \mathrm{Kg})\end{array}$ & $\begin{array}{c}7749 \mathrm{D} \\
960822-036 \\
12.6 \\
\\
50.9 \\
4 \quad 9 \quad 1 \\
\\
\\
1.36 \\
15700 \\
15700 \\
<15\end{array}$ & $\begin{array}{c}10224 \\
000509-002 \\
11.0 \\
59.5 \\
4 \emptyset 6 \\
282 \\
124 \\
1.331 \\
2 \varnothing 9 \emptyset \emptyset \\
11100 \\
9800\end{array}$ & $\begin{array}{c}12057 \\
010716-011 \\
11.8 \\
55.4 \\
446 \\
\\
\\
1.359 \\
2330 \emptyset \\
11000 \\
12300\end{array}$ & $\begin{array}{l}15 \\
15 \\
15\end{array}$ \\
\hline \multicolumn{6}{|c|}{ RCRA Metals $( \pm 10 \%)$} \\
\hline $\begin{array}{ll}\mathrm{Ag}^{\mathrm{h}} & (100)^{\prime} \\
\mathrm{As} & (100) \\
\mathrm{Ba} & (2000) \\
\mathrm{Cd} & (20) \\
\mathrm{Cr} & (100) \\
\mathrm{Hg} & (4) \\
\mathrm{Ni} & (1000) \\
\mathrm{Pb} & (100) \\
\mathrm{Se} & (20) \\
\mathrm{Tl} & (18)\end{array}$ & $\begin{array}{l}(\mathrm{mg} / \mathrm{Kg}) \\
(\mathrm{mg} / \mathrm{Kg}) \\
(\mathrm{mg} / \mathrm{Kg}) \\
(\mathrm{mg} / \mathrm{Kg}) \\
(\mathrm{mg} / \mathrm{Kg}) \\
(\mathrm{mg} / \mathrm{Kg}) \\
(\mathrm{mg} / \mathrm{Kg}) \\
(\mathrm{mg} / \mathrm{Kg}) \\
(\mathrm{mg} / \mathrm{Kg}) \\
(\mathrm{mg} / \mathrm{Kg})\end{array}$ & $\begin{array}{c}<1.8 \\
<1.3 \\
105 \\
11.9 \\
92.1 \\
73.2 \\
56.8 \\
442 \\
<1.3 \\
<1.3\end{array}$ & $\begin{array}{c}<\mathbf{0 . 9} \\
73.8 \\
26 . \emptyset \\
190 \\
49.1 \\
82.3 \\
454 \\
<0.9 \\
<0.9\end{array}$ & $\begin{array}{l}24.8 \\
<1.7 \\
106 \\
32.5 \\
197 \\
106 \\
85.8 \\
679 \\
<0.8 \\
<4\end{array}$ & $\begin{array}{l}0.006 \\
0.011 \\
0.001 \\
0.111 \\
0.008 \\
0.011 \\
0.065 \\
0.341 \\
0.005 \\
0.005\end{array}$ \\
\hline \multicolumn{6}{|c|}{ Process metals $( \pm 10 \%)$} \\
\hline $\begin{array}{l}\mathrm{Al} \\
\mathrm{B} \\
\mathrm{Be} \\
\mathrm{Ca} \\
\mathrm{co} \\
\mathrm{cu} \\
\mathrm{Cs}^{j} \\
\mathrm{Fe} \\
\mathrm{K} \\
\mathrm{Mg} \\
\mathrm{Mn} \\
\mathrm{Mo} \\
\mathrm{Na} \\
\mathrm{P} \\
\mathrm{Sb} \\
\mathrm{Si} \\
\mathrm{Sr} \\
\mathrm{Th} \\
\mathrm{Ti} \\
\mathrm{U} \\
\mathrm{V} \\
\mathrm{Zn} \\
\mathrm{Zr}\end{array}$ & $\begin{array}{l}(\mathrm{mg} / \mathrm{Kg}) \\
(\mathrm{mg} / \mathrm{Kg}) \\
(\mathrm{mg} / \mathrm{Kg}) \\
(\mathrm{mg} / \mathrm{Kg}) \\
(\mathrm{mg} / \mathrm{Kg}) \\
(\mathrm{mg} / \mathrm{Kg}) \\
(\mathrm{mg} / \mathrm{Kg}) \\
(\mathrm{mg} / \mathrm{Kg}) \\
(\mathrm{mg} / \mathrm{Kg}) \\
(\mathrm{mg} / \mathrm{Kg}) \\
(\mathrm{mg} / \mathrm{Kg}) \\
(\mathrm{mg} / \mathrm{Kg}) \\
(\mathrm{mg} / \mathrm{Kg}) \\
(\mathrm{mg} / \mathrm{Kg}) \\
(\mathrm{mg} / \mathrm{Kg}) \\
(\mathrm{mg} / \mathrm{Kg}) \\
(\mathrm{mg} / \mathrm{Kg}) \\
(\mathrm{mg} / \mathrm{Kg}) \\
(\mathrm{mg} / \mathrm{Kg}) \\
(\mathrm{mg} / \mathrm{Kg}) \\
(\mathrm{mg} / \mathrm{Kg}) \\
(\mathrm{mg} / \mathrm{Kg}) \\
(\mathrm{mg} / \mathrm{Kg})\end{array}$ & $\begin{array}{c}581 \emptyset \\
3.76 \\
6.91 \\
5080 \emptyset \\
5.86 \\
37 . \emptyset \\
0.857 \\
181 \emptyset \\
885 \emptyset \\
765 \emptyset \\
14 \emptyset \\
\\
5210 \emptyset \\
185 \emptyset \\
114 \\
889 \emptyset \\
325 \\
925 \emptyset \\
766 \emptyset \\
3.85 \\
285\end{array}$ & $\begin{array}{c}261 \emptyset \\
7.34 \\
6.4 \emptyset \\
5610 \emptyset \\
28.1 \\
51.4 \\
<\emptyset . \emptyset 9 \\
216 \emptyset \\
982 \emptyset \\
11500 \\
616 \\
37 . \emptyset \\
42 \emptyset 0 \emptyset \\
<50 \\
267 \emptyset \\
251 \\
896 \emptyset \\
\\
3060 \emptyset \\
<1.2 \\
623\end{array}$ & $\begin{array}{c}658 \emptyset \\
3.65 \\
7.83 \\
6 \emptyset \emptyset \emptyset \emptyset \\
42.5 \\
61.6 \\
<4 \\
253 \emptyset \\
923 \emptyset \\
106 \emptyset \emptyset \\
469 \\
91.6 \\
464 \emptyset \emptyset \\
\\
<4 \\
563 \emptyset \\
234 \\
10100 \\
32.5 \\
18 \emptyset \emptyset \emptyset \\
<1 \\
458 \\
331\end{array}$ & 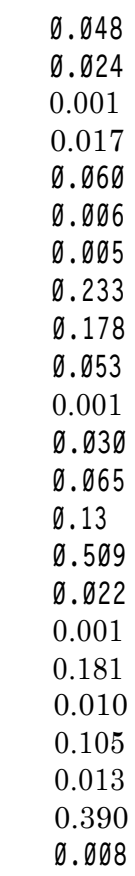 \\
\hline
\end{tabular}




\begin{tabular}{|c|c|c|c|c|c|}
\hline \multicolumn{2}{|l|}{$\begin{array}{l}\text { Characteristic } \\
\text { (Analysis) }\end{array}$} & $\begin{array}{c}1996 \\
W-25 \mathrm{~S}\end{array}$ & $\begin{array}{c}2000 \\
\mathbf{W}-25 \text { S }\end{array}$ & $\begin{array}{c}2001 \\
\mathbf{W}-25 \mathrm{~S}\end{array}$ & IDL' \\
\hline \multicolumn{6}{|c|}{ Semi-quantitative metals by ICP-MS $( \pm 30-50 \%, *$ indicates data from water leach) } \\
\hline $\begin{array}{l}\mathrm{Au} \text {, gold } \\
\mathrm{Bi} \text {, bismuth } \\
\mathrm{Ce} \text {, cerium } \\
\mathrm{Er} \text {, erbium } \\
\mathrm{Eu} \text {, europium } \\
\mathrm{Ga} \text {, gallium } \\
\mathrm{Gd} \text {, gadolinium } \\
\mathrm{Ho} \text {, holmium } \\
\mathrm{I} \text {, iodine } \\
\mathrm{La} \text {, lanthanum } \\
\mathrm{Li} \text {, lithium } \\
\mathrm{Mo} \text {, molybdenum } \\
\mathrm{Nb} \text {, niobium } \\
\mathrm{Rb} \text {, rubidium } \\
\mathrm{Sn} \text {, tin } \\
\mathrm{Ti} \text {, titanium } \\
\mathrm{W} \text {, tungsten } \\
\mathrm{Zr} \text {, zirconium }\end{array}$ & $\begin{array}{l}(\mathrm{mg} / \mathrm{Kg}) \\
(\mathrm{mg} / \mathrm{Kg}) \\
(\mathrm{mg} / \mathrm{Kg}) \\
(\mathrm{mg} / \mathrm{Kg}) \\
(\mathrm{mg} / \mathrm{Kg}) \\
(\mathrm{mg} / \mathrm{Kg}) \\
(\mathrm{mg} / \mathrm{Kg}) \\
(\mathrm{mg} / \mathrm{Kg}) \\
(\mathrm{mg} / \mathrm{Kg}) \\
(\mathrm{mg} / \mathrm{Kg}) \\
(\mathrm{mg} / \mathrm{Kg}) \\
(\mathrm{mg} / \mathrm{Kg}) \\
(\mathrm{mg} / \mathrm{Kg}) \\
(\mathrm{mg} / \mathrm{Kg}) \\
(\mathrm{mg} / \mathrm{Kg}) \\
(\mathrm{mg} / \mathrm{Kg}) \\
(\mathrm{mg} / \mathrm{Kg}) \\
(\mathrm{mg} / \mathrm{Kg})\end{array}$ & $\begin{array}{c}0.28 \\
250 \\
9.4 \\
0.02 \\
2.1 \\
8.1 \\
1.7 \\
2.0 \\
* 12 \\
18 \\
* 33 \\
* 2.0 \\
0.72 \\
* 1.0 \\
18 \\
47 \\
0.61 \\
16\end{array}$ & & 15.5 & - \\
\hline \multicolumn{6}{|c|}{ Anions by ion chromatography in water wash of sludge $( \pm 10 \%)$} \\
\hline \multicolumn{6}{|l|}{ Inorganic } \\
\hline $\begin{array}{l}\text { Bromide } \\
\text { Chloride } \\
\text { Chromate } \\
\text { Fluoride } \\
\text { Nitrate } \\
\text { Nitrite } \\
\text { Phosphate } \\
\text { Sulphate } \\
\text { Organic } \\
\text { Acetate } \\
\text { Citrate } \\
\text { Forrnate } \\
\text { Oxalate } \\
\text { Phthalate }\end{array}$ & $\begin{array}{l}(\mathrm{mg} / \mathrm{Kg}) \\
(\mathrm{mg} / \mathrm{Kg}) \\
(\mathrm{mg} / \mathrm{Kg}) \\
(\mathrm{mg} / \mathrm{Kg}) \\
(\mathrm{mg} / \mathrm{Kg}) \\
(\mathrm{mg} / \mathrm{Kg}) \\
(\mathrm{mg} / \mathrm{Kg}) \\
(\mathrm{mg} / \mathrm{Kg}) \\
(\mathrm{mg} / \mathrm{Kg}) \\
(\mathrm{mg} / \mathrm{Kg}) \\
(\mathrm{mg} / \mathrm{Kg}) \\
(\mathrm{mg} / \mathrm{Kg}) \\
(\mathrm{mg} / \mathrm{Kg})\end{array}$ & $\begin{array}{c}<50 \\
2110 \\
95.5 \\
118 \\
162000 \\
4967 \\
<20 \\
1750 \\
\\
318 \\
<20 \\
247 \\
521 \\
<20\end{array}$ & $\begin{array}{c}<50 \\
1630 \\
29.5 \\
251 \\
76600 \\
3190 \\
<10 \\
2590 \\
\\
919 \\
25.5 \\
130 \\
643 \\
18.4\end{array}$ & $\begin{array}{c}128 \\
1720 \\
89.1 \\
155 \\
120000 \\
5440 \\
<10 \\
2810 \\
\\
641 \\
52.7 \\
174 \\
238 \\
<5\end{array}$ & $\begin{array}{l}0.05 \\
0.05 \\
0.05 \\
0.05 \\
0.10 \\
0.10 \\
0.20 \\
0.10 \\
\\
0.05 \\
0.50 \\
0.05 \\
0.05 \\
0.05\end{array}$ \\
\hline
\end{tabular}




\begin{tabular}{|c|c|c|c|c|c|}
\hline $\begin{array}{l}\text { Characteristic } \\
\text { (Analysis) }\end{array}$ & & $\begin{array}{c}1996 \\
W-25 \mathrm{~S}\end{array}$ & $\begin{array}{c}2000 \\
\mathbf{W}-25 \mathbf{S}\end{array}$ & $\begin{array}{c}2001 \\
\mathbf{W}-25 \mathbf{S}\end{array}$ & IDL' \\
\hline \multicolumn{6}{|c|}{ Beta/gamma emitters $( \pm 10 \%)$} \\
\hline $\begin{array}{l}\text { Gross beta } \\
{ }^{59} \mathrm{Ni} \\
{ }^{53} \mathrm{Ni} \\
{ }^{50} \mathrm{Co} \\
{ }^{90} \mathrm{Sr}{ }^{90} \mathrm{Y} \\
{ }^{99} \mathrm{Tc} \\
{ }^{129} \mathrm{I} \\
{ }^{134} \mathrm{Cs} \\
{ }^{137} \mathrm{Cs} \\
{ }^{151} \mathrm{Sm} \\
{ }^{152} \mathrm{Eu} \\
{ }^{154} \mathrm{Eu} \\
{ }^{155} \mathrm{Eu} \\
{ }^{227} \mathrm{Ac} \\
{ }^{241} \mathrm{Pu}\end{array}$ & $\begin{array}{l}\text { @ } \mathrm{q} / \mathrm{g}) \\
(\mathrm{Bq} / \mathrm{g}) \\
(\mathrm{Bq} / \mathrm{g}) \\
(\mathrm{Bq} / \mathrm{g}) \\
(\mathrm{Bq} / \mathrm{g}) \\
(\mathrm{Bq} / \mathrm{g}) \\
(\mathrm{Bq} / \mathrm{g}) \\
(\mathrm{Bq} / \mathrm{g}) \\
(\mathrm{Bq} / \mathrm{g}) \\
(\mathrm{Bq} / \mathrm{g}) \\
(\mathrm{Bq} / \mathrm{g}) \\
(\mathrm{Bq} / \mathrm{g}) \\
(\mathrm{Bq} / \mathrm{g}) \\
(\mathrm{Bq} / \mathrm{g}) \\
(\mathrm{Bq} / \mathrm{g})\end{array}$ & $\begin{array}{r}8.3 \mathrm{e}+06 \\
<2.5 \mathrm{e}+01 \\
3.4 \mathrm{e}+03 \\
2.5 \mathrm{e}+04 \\
3.2 \mathrm{e}+06 \\
1.0 \mathrm{e}+02 \\
\\
6.0 \mathrm{e}+03 \\
4.7 \mathrm{e}+05 \\
<5.5 \mathrm{e}+02 \\
7.1 \mathrm{e}+04 \\
3.7 \mathrm{e}+04 \\
8.4 \mathrm{e}+03 \\
<5.3 \mathrm{e}+03 \\
2.6 \mathrm{e}+04\end{array}$ & $\begin{array}{r}4.3 e+06 \\
3.5 e+04 \\
1.3 e+06 \\
\\
4.0 \mathrm{e}+05 \\
6.0 \mathrm{e}+05 \\
<7.1 \mathrm{e}+04 \\
2.8 \mathrm{e}+04\end{array}$ & $\begin{array}{r}5.9 e+06 \\
\\
2.2 e+04 \\
2.3 e+06 \\
9.1 e+02 \\
<1.8 e+03 \\
3.5 e+05 \\
\\
3.0 e+05 \\
1.0 e+05 \\
2.2 e+04\end{array}$ & - \\
\hline \multicolumn{6}{|c|}{ Alpha emitters $( \pm 10 \%)$} \\
\hline $\begin{array}{l}\text { Gross alpha } \\
{ }^{232} \mathrm{Th} \\
{ }^{233} \mathrm{U} \\
{ }^{234} \mathrm{U} \\
{ }^{235} \mathrm{U} \\
{ }^{238} \mathrm{U} \\
{ }^{23} \mathrm{~Np} \\
{ }^{244} \mathrm{Am} \\
{ }^{250} \mathrm{Cf} \\
{ }^{252} \mathrm{Cf} \\
{ }^{\mathrm{T} o t a l ~} \mathrm{Pu} \text { alpha } \\
{ }^{238} \mathrm{Pu} \\
{ }^{239} \mathrm{Pu} /{ }^{240} \mathrm{Pu} \\
{ }^{242} \mathrm{Pu} \\
\mathrm{TRU} \text { activity } \\
\mathrm{Pu}+\mathrm{Am} \text { (3700) }\end{array}$ & $\begin{array}{l}(\mathrm{Bq} / \mathrm{g}) \\
(\mathrm{Bq} / \mathrm{g}) \\
(\mathrm{Bq} / \mathrm{g}) \\
(\mathrm{Bq} / \mathrm{g}) \\
(\mathrm{Bq} / \mathrm{g}) \\
(\mathrm{Bq} / \mathrm{g}) \\
(\mathrm{Bq} / \mathrm{g}) \\
(\mathrm{Bq} / \mathrm{g}) \\
(\mathrm{Bq} / \mathrm{g}) \\
(\mathrm{Bq} / \mathrm{g}) \\
(\mathrm{Bq} / \mathrm{g}) \\
(\mathrm{Bq} / \mathrm{g}) \\
(\mathrm{Bq} / \mathrm{g}) \\
(\mathrm{Bq} / \mathrm{g}) \\
\\
(\mathrm{Bq} / \mathrm{g})\end{array}$ & $\begin{array}{c}83000 \\
38 \\
2800 \\
100 \\
3.2 \\
95 \\
10 \\
9300 \\
58000 \\
<100 \\
<100 \\
\\
13000 \\
7700 \\
4900 \\
\end{array}$ & $\begin{array}{c}130000 \\
36 \\
7200 \\
28 \\
7.9 \\
380 \\
\\
10300 \\
90500\end{array}$ & $\begin{array}{c}110000 \\
41 \\
3900 \\
98 \\
5.9 \\
230 \\
\\
8600 \\
75000\end{array}$ & \\
\hline \multicolumn{6}{|c|}{ Uranium isotopics by ICP-MS $( \pm 2 \%)$} \\
\hline $\begin{array}{l}{ }^{233} \mathrm{U} \\
{ }^{234} \mathrm{U} \\
{ }^{235} \mathrm{U} \\
{ }^{236} \mathrm{U} \\
{ }^{238} \mathrm{U} \\
{ }^{233} \mathrm{U} / \mathrm{MS} \\
{ }^{235} \mathrm{U} / \mathrm{MS} \\
{ }^{238} \mathrm{U} /{ }^{235} \mathrm{U} \text { FEM }\end{array}$ & $\begin{array}{l}(\text { atom \%) } \\
(\text { atom \%) } \\
(\text { atom \%) } \\
(\text { atom \%) } \\
(\text { atom \%) } \\
(\mathrm{mg} / \mathrm{Kg}) \\
(\mathrm{mg} / \mathrm{Kg})\end{array}$ & $\begin{array}{c}0.103 \\
0.006 \\
0.597 \\
0.006 \\
99.289 \\
\\
7.72 \\
45.2 \\
137\end{array}$ & $\begin{array}{r}0.0674 \\
0.0004 \\
0.3670 \\
0.0048 \\
99.5604 \\
\\
20.2 \\
111 \\
221\end{array}$ & $\begin{array}{l}0.0614 \\
0.0024 \\
0.4147 \\
0.0058 \\
99.5157 \\
\\
10.8 \\
73.7 \\
203\end{array}$ & $\begin{array}{l}0.001 \\
0.001 \\
0.001 \\
0.001 \\
0.001\end{array}$ \\
\hline
\end{tabular}




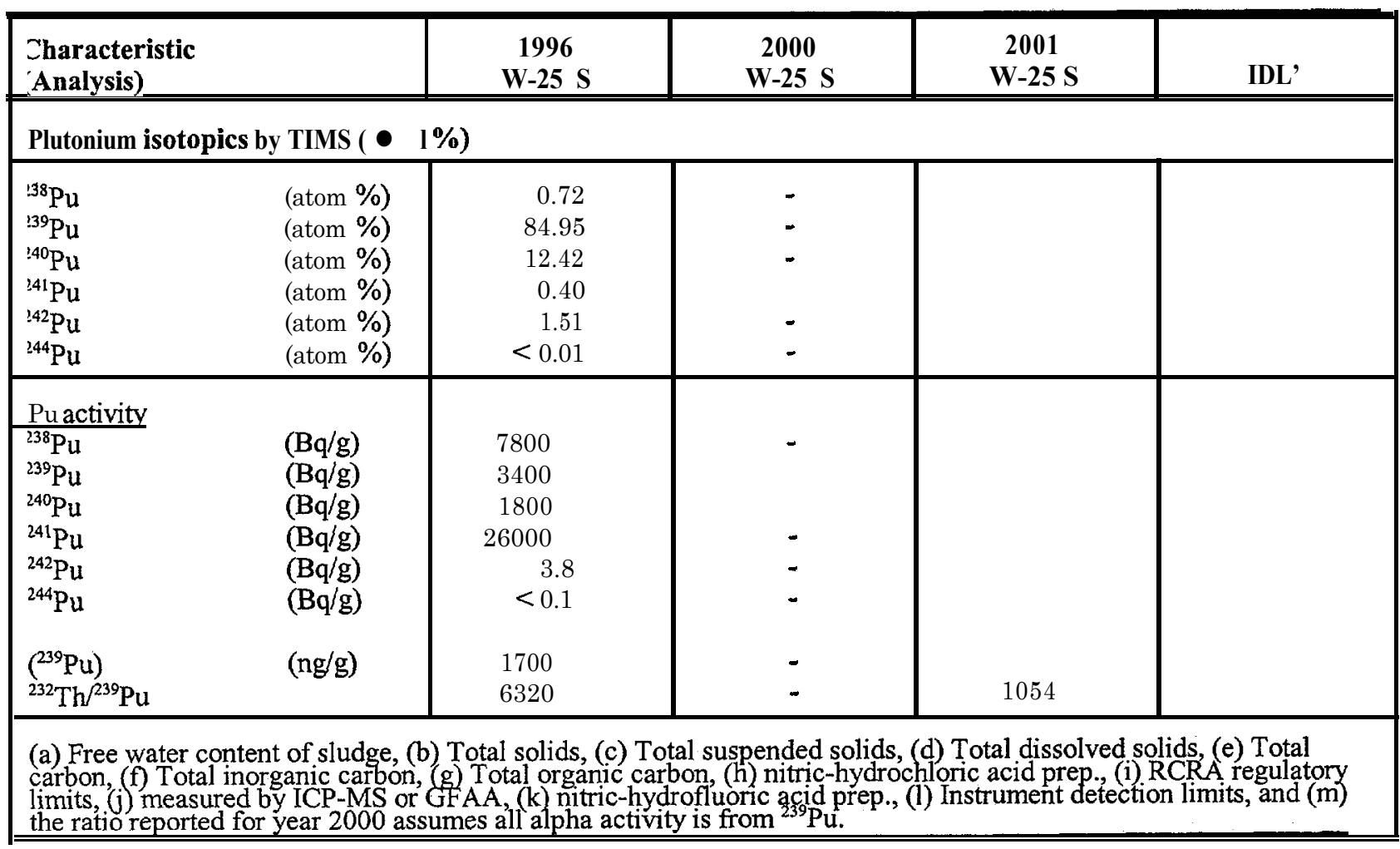


Table 4 Analytical Data for Sludge in Tanks W-26

\begin{tabular}{|c|c|c|c|c|c|}
\hline \multicolumn{2}{|l|}{$\begin{array}{l}\text { Characteristic } \\
\text { (Analysis) }\end{array}$} & $\begin{array}{c}1996 \\
W-26 \mathrm{~S}\end{array}$ & $\begin{array}{c}2000 \\
\mathbf{W}-26 \mathbf{S}\end{array}$ & $\begin{array}{c}2001 \\
\mathbf{W}-26 \mathbf{S}\end{array}$ & I D L , \\
\hline \multicolumn{6}{|c|}{ Physical properties and miscellaneous data } \\
\hline $\begin{array}{l}\text { Request number } \\
\text { Sample number } \\
\text { pH } \\
\text { Water }^{\mathbf{a}} \\
\text { TS }^{\mathbf{b}} \\
\text { TSS }^{\mathrm{c}} \\
\text { TDS }^{\text {d }} \\
\text { Bulk density } \\
\text { TC" }^{\prime} \\
\text { TIC' } \\
\text { TOC }\end{array}$ & $\begin{array}{l}(\%) \\
(\mathrm{mg} / \mathrm{g}) \\
(\mathrm{mg} / \mathrm{g}) \\
(\mathrm{mg} / \mathrm{g}) \\
(\mathrm{g} / \mathrm{mL}) \\
(\mathrm{mg} / \mathrm{Kg}) \\
(\mathrm{mg} / \mathrm{Kg}) \\
(\mathrm{mg} / \mathrm{Kg})\end{array}$ & $\begin{array}{c}7749 \mathrm{E} \\
960830-044 \\
9.7 \\
50.9 \\
491 \\
\\
\\
1.38 \\
13500 \\
11600 \\
1900\end{array}$ & $\begin{array}{c}10224 \\
000509-003 \\
9.1 \\
56.1 \\
439 \\
285 \\
154 \\
1.363 \\
10300 \\
3790 \\
6510\end{array}$ & $\begin{array}{c}12057 \\
010716-012 \\
9.4 \\
52.9 \\
471 \\
\\
1.675 \\
11300 \\
3000 \\
8300\end{array}$ & $\begin{array}{r}- \\
15 \\
15 \\
15\end{array}$ \\
\hline \multicolumn{6}{|c|}{ RCRA Metals $( \pm 10 \%)$} \\
\hline $\begin{array}{ll}\mathrm{Ag}^{\mathbf{h}} & (100)^{\prime} \\
\mathrm{As} & (100) \\
\mathrm{Ba} & (2000) \\
\mathrm{Cd} & (20) \\
\mathrm{Cr} & (100) \\
\mathrm{Hg} & (4) \\
\mathrm{Ni} & (1000) \\
\mathrm{Pb} & (100) \\
\mathrm{Se} & (20) \\
\mathrm{Tl} & (18)\end{array}$ & $\begin{array}{l}(\mathrm{mg} / \mathrm{Kg}) \\
(\mathrm{mg} / \mathrm{Kg}) \\
(\mathrm{mg} / \mathrm{Kg}) \\
(\mathrm{mg} / \mathrm{Kg}) \\
(\mathrm{mg} / \mathrm{Kg}) \\
(\mathrm{mg} / \mathrm{Kg}) \\
(\mathrm{mg} / \mathrm{Kg}) \\
(\mathrm{mg} / \mathrm{Kg}) \\
(\mathrm{mg} / \mathrm{Kg}) \\
(\mathrm{mg} / \mathrm{Kg})\end{array}$ & $\begin{array}{c}<1.9 \\
<1.4 \\
63.1 \\
19.8 \\
74.4 \\
12.7 \\
42.8 \\
212 \\
<1.4 \\
<1.4\end{array}$ & $\begin{array}{c}<0.9 \\
77.9 \\
21.9 \\
153 \\
58.3 \\
74.5 \\
331 \\
<0.9 \\
<0.9\end{array}$ & $\begin{array}{c}<1.8 \\
134 \\
48.2 \\
212 \\
89.5 \\
94.3 \\
635 \\
<0.8 \\
<4\end{array}$ & $\begin{array}{r}0.006 \\
0.011 \\
0.001 \\
0.111 \\
0.008 \\
0.011 \\
0.065 \\
0.341 \\
0.005 \\
0.005\end{array}$ \\
\hline \multicolumn{6}{|c|}{ Process metals $( \pm 10 \%)$} \\
\hline $\begin{array}{l}\mathrm{Al} \\
\mathrm{B} \\
\mathrm{Be} \\
\mathrm{Ca} \\
\mathrm{co} \\
\mathrm{cu} \\
\mathrm{Cs}^{\mathrm{j}} \\
\mathrm{Fe} \\
\mathrm{K} \\
\mathrm{Mg} \\
\mathrm{Mn} \\
\mathrm{Mo} \\
\mathrm{N} \\
\mathrm{P} \\
\mathrm{Sb} \\
\mathrm{Si} \\
\mathrm{Sr} \\
\mathrm{Th} \\
\mathrm{Ti} \\
\mathrm{U} \\
\mathrm{V} \\
\mathrm{Zn} \\
\mathrm{Zr}\end{array}$ & $\begin{array}{l}(\mathrm{mg} / \mathrm{Kg}) \\
(\mathrm{mg} / \mathrm{Kg}) \\
(\mathrm{mg} / \mathrm{Kg}) \\
(\mathrm{mg} / \mathrm{Kg}) \\
(\mathrm{mg} / \mathrm{Kg}) \\
(\mathrm{mg} / \mathrm{Kg}) \\
(\mathrm{mg} / \mathrm{Kg}) \\
(\mathrm{mg} / \mathrm{Kg}) \\
(\mathrm{mg} / \mathrm{Kg}) \\
(\mathrm{mg} / \mathrm{Kg}) \\
(\mathrm{mg} / \mathrm{Kg}) \\
(\mathrm{mg} / \mathrm{Kg}) \\
(\mathrm{mg} / \mathrm{Kg}) \\
(\mathrm{mg} / \mathrm{Kg}) \\
(\mathrm{mg} / \mathrm{Kg}) \\
(\mathrm{mg} / \mathrm{Kg}) \\
(\mathrm{mg} / \mathrm{Kg}) \\
(\mathrm{mg} / \mathrm{Kg}) \\
(\mathrm{mg} / \mathrm{Kg}) \\
(\mathrm{mg} / \mathrm{Kg}) \\
(\mathrm{mg} / \mathrm{Kg}) \\
(\mathrm{mg} / \mathrm{Kg}) \\
(\mathrm{mg} / \mathrm{Kg})\end{array}$ & $\begin{array}{c}1980 \\
11.3 \\
1.85 \\
45900 \\
2.69 \\
29.0 \\
1.53 \\
1010 \\
25300 \\
14700 \\
102 \\
\\
48900 \\
1070 \\
52.8 \\
2100 \\
254 \\
3280 \\
\\
19400 \\
2.32 \\
405\end{array}$ & $\begin{array}{c}7130 \\
10.6 \\
5.63 \\
43200 \\
21.1 \\
70.6 \\
0.729 \\
2380 \\
18400 \\
12800 \\
180 \\
46.4 \\
40500 \\
\\
<50 \\
7470 \\
195 \\
4330 \\
- \\
36900 \\
<1.2 \\
360\end{array}$ & $\begin{array}{c}7590 \\
6.87 \\
5.33 \\
61700 \\
41.5 \\
63.7 \\
<4 \\
2740 \\
17400 \\
14500 \\
278 \\
72.4 \\
39700 \\
\\
<4 \\
8330 \\
216 \\
5880 \\
30.2 \\
30200 \\
<1 \\
440 \\
200\end{array}$ & $\begin{array}{l}0.048 \\
0.024 \\
0.001 \\
0.017 \\
0.060 \\
0.006 \\
0.005 \\
0.233 \\
0.178 \\
0.053 \\
0.001 \\
0.030 \\
0.065 \\
0.13 \\
0.509 \\
0.022 \\
0.001 \\
0.181 \\
0.010 \\
0.105 \\
0.013 \\
0.390 \\
0.008\end{array}$ \\
\hline
\end{tabular}




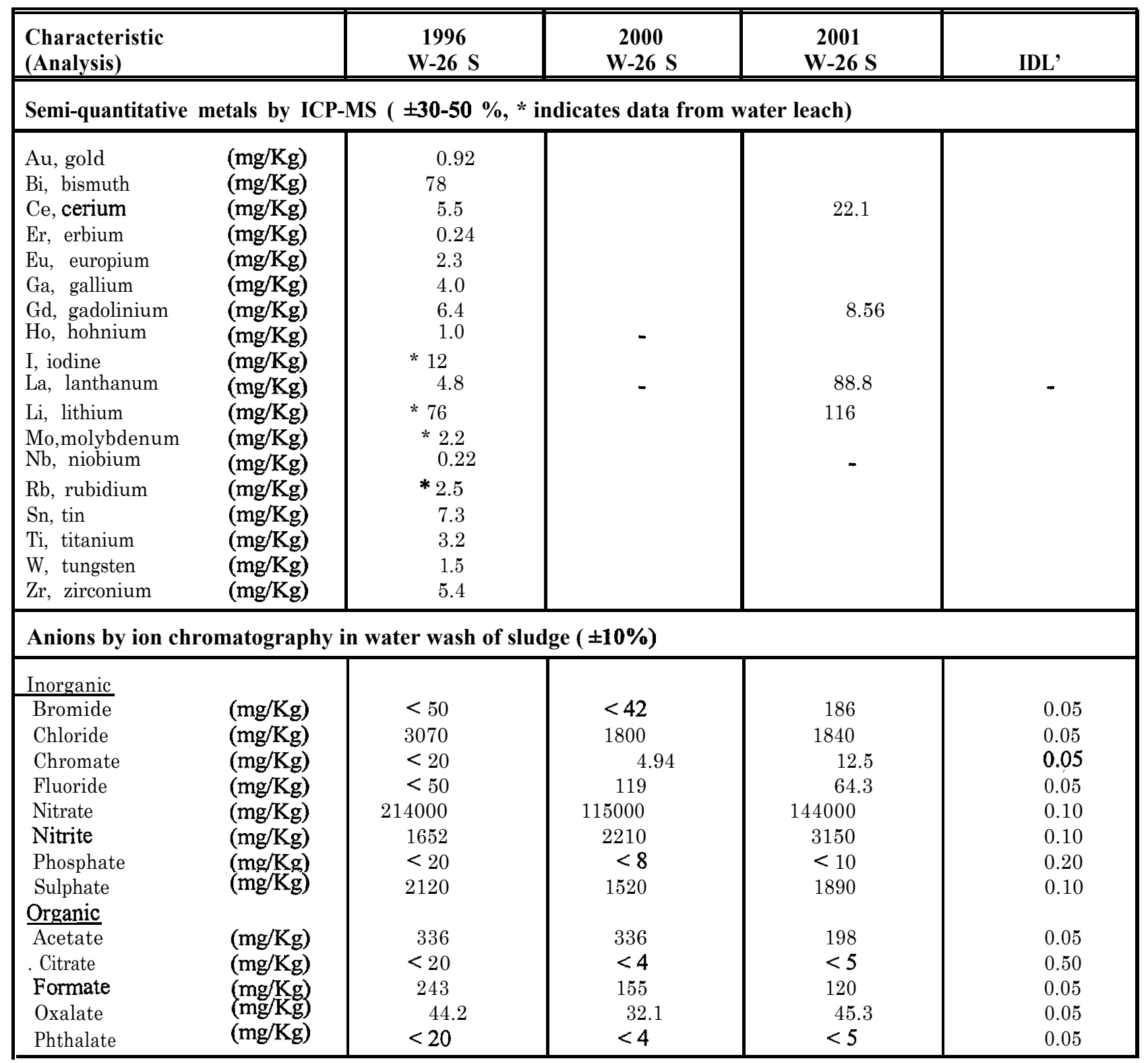




\begin{tabular}{|c|c|c|c|c|c|}
\hline \multicolumn{2}{|l|}{$\begin{array}{l}\text { Tharacteristic } \\
\text { Analysis) }\end{array}$} & $\begin{array}{c}1996 \\
\text { W-26 S } \\
\end{array}$ & $\begin{array}{c}2000 \\
W-26 \mathrm{~S} \\
\end{array}$ & $\begin{array}{c}2001 \\
\text { W-26 S } \\
\end{array}$ & IDL' \\
\hline \multicolumn{6}{|c|}{ 3eta/gamma emitters ( $\pm 10 \%$ ) } \\
\hline $\begin{array}{l}\text { Irross beta } \\
-\mathrm{Ni}^{\text {s }} \\
{ }^{3} \mathrm{Ni} \\
{ }^{0} \mathrm{Co} \\
{ }^{0} \mathrm{Sr}{ }^{90} \mathrm{Y} \\
{ }^{9} \mathrm{Tc} \\
{ }^{29} \mathrm{I} \\
{ }^{34} \mathrm{Cs} \\
{ }^{37} \mathrm{Cs} \\
{ }^{51} \mathrm{Sm} \\
{ }^{52} \mathrm{Eu} \\
{ }^{54} \mathrm{Eu} \\
{ }^{55} \mathrm{Eu} \\
{ }^{22} \mathrm{Ac} \\
{ }^{! 41} \mathrm{Pu}\end{array}$ & $\begin{array}{l}(\mathrm{Bq} / \mathrm{g}) \\
(\mathrm{Bq} / \mathrm{g}) \\
(\mathrm{Bq} / \mathrm{g}) \\
(\mathrm{Bq} / \mathrm{g}) \\
(\mathrm{Bq} / \mathrm{g}) \\
(\mathrm{Bq} / \mathrm{g}) \\
(\mathrm{Bq} / \mathrm{g}) \\
(\mathrm{Bq} / \mathrm{g}) \\
(\mathrm{Bq} / \mathrm{g}) \\
(\mathrm{Bq} / \mathrm{g}) \\
(\mathrm{Bq} / \mathrm{g}) \\
(\mathrm{Bq} / \mathrm{g}) \\
(\mathrm{Bq} / \mathrm{g}) \\
\end{array}$ & $\begin{array}{r}3.5 \mathrm{e}+06 \\
<3.0 \mathrm{e}+01 \\
4.0 \mathrm{e}+03 \\
5.8 \mathrm{e}+04 \\
7.1 \mathrm{e}+05 \\
1.2 \mathrm{e}+03 \\
- \\
1.2 \mathrm{e}+04 \\
8.9 \mathrm{e}+05 \\
<5.8 \mathrm{e}+02 \\
6.4 \mathrm{e}+05 \\
2.9 \mathrm{e}+05 \\
6.3 \mathrm{e}+04 \\
<9.3 \mathrm{e}+03 \\
1.5 \mathrm{e}+04 \\
\end{array}$ & $\begin{array}{l}4.0 \mathrm{e}+06 \\
- \\
- \\
3.1 \mathrm{e}+04 \\
1.0 \mathrm{e}+06 \\
\\
\\
8.1 \mathrm{e}+05 \\
3.9 \mathrm{e}+05 \\
1.7 \mathrm{e}+05 \\
<1.8 \mathrm{e}+04 \\
- \\
-\end{array}$ & $\begin{array}{l}4.4 \mathrm{e}+06 \\
- \\
3.3 \mathrm{e}+04 \\
1.3 \mathrm{e}+06 \\
1.1 \mathrm{e}+03 \\
<2.8 \mathrm{e}+03 \\
6.3 \mathrm{e}+05 \\
\\
4.6 \mathrm{e}+05 \\
1.9 \mathrm{e}+05 \\
5.6 \mathrm{e}+04\end{array}$ & - \\
\hline \multicolumn{6}{|c|}{ Alpha emitters $( \pm 10 \%)$} \\
\hline $\begin{array}{l}\text { Gross alpha } \\
{ }^{132} \mathrm{Th} \\
{ }^{133} \mathrm{U} \\
{ }^{234} \mathrm{U} \\
{ }^{235} \mathrm{U} \\
{ }^{238} \mathrm{U} \\
{ }^{237} \mathrm{~Np} \\
{ }^{241} \mathrm{Am} \\
{ }^{244} \mathrm{Cm} \\
{ }^{250} \mathrm{Cf} \\
{ }^{252} \mathrm{Cf} \\
{ }^{238} \mathrm{Pu} \text { Pu alpha } \\
{ }^{239} \mathrm{Pu} /{ }^{240} \mathrm{Pu} \\
{ }^{242} \mathrm{Pu} \\
\text { TRU activity } \\
\mathrm{Pu}+\mathrm{Am} \mathrm{(3700)}\end{array}$ & $\begin{array}{l}(\mathrm{Bq} / \mathrm{g}) \\
(\mathrm{Bq} / \mathrm{g}) \\
(\mathrm{Bq} / \mathrm{g}) \\
(\mathrm{Bq} / \mathrm{g}) \\
(\mathrm{Bq} / \mathrm{g}) \\
(\mathrm{Bq} / \mathrm{g}) \\
(\mathrm{Bq} / \mathrm{g}) \\
(\mathrm{Bq} / \mathrm{g}) \\
(\mathrm{Bq} / \mathrm{g}) \\
(\mathrm{Bq} / \mathrm{g}) \\
(\mathrm{Bq} / \mathrm{g}) \\
(\mathrm{Bq} / \mathrm{g}) \\
(\mathrm{Bq} / \mathrm{g}) \\
(\mathrm{Bq} / \mathrm{g}) \\
(\mathrm{Bq} / \mathrm{g})\end{array}$ & $\begin{array}{c}52000 \\
13 \\
10000 \\
180 \\
4.0 \\
240 \\
2 \\
3900 \\
28000 \\
<100 \\
<100 \\
\\
7600 \\
5300 \\
2300 \\
\end{array}$ & $\begin{array}{r}48000 \\
18 \\
10500 \\
640 \\
12 \\
460 \\
- \\
4880 \\
28000 \\
- \\
- \\
9100 \\
5200 \\
3900\end{array}$ & $\begin{array}{c}68000 \\
18 \\
8200 \\
210 \\
8.8 \\
370 \\
- \\
5000 \\
40000 \\
- \\
- \\
13000 \\
8300 \\
5100 \\
\\
\\
\end{array}$ & $\begin{array}{l}- \\
- \\
- \\
- \\
- \\
- \\
- \\
- \\
- \\
-\end{array}$ \\
\hline \multicolumn{6}{|c|}{ Uranium isotopics by ICP-MS $( \pm 2 \%)$} \\
\hline $\begin{array}{l}{ }^{233} \mathrm{U} \\
{ }^{234} \mathrm{U} \\
{ }^{235} \mathrm{U} \\
{ }^{236} \mathrm{U} \\
{ }^{238} \mathrm{U} \\
{ }^{233} \mathrm{U} / \mathrm{MS} \\
{ }^{235} \mathrm{U} / \mathrm{MS} \\
{ }^{238} \mathrm{U} /{ }^{235} \mathrm{U} F \mathrm{FE}\end{array}$ & $\begin{array}{l}(\text { atom \%) } \\
(\text { atom \%) } \\
(\text { atom \%) } \\
(\text { atom \%) } \\
(\text { atom \%) } \\
(\mathrm{mg} / \mathrm{Kg}) \\
(\mathrm{mg} / \mathrm{Kg})\end{array}$ & $\begin{array}{l}0.152 \\
0.004 \\
0.296 \\
0.006 \\
99.543 \\
\\
28.9 \\
56.7 \\
202\end{array}$ & $\begin{aligned} & 0.0814 \\
& 0.0076 \\
& 0.4800 \\
&<0.0001 \\
& 99.4310 \\
& \\
& 29.4 \\
& 175 \\
& 171\end{aligned}$ & $\begin{array}{l}0.0773 \\
0.0031 \\
0.4157 \\
0.0040 \\
99.4999 \\
\\
22.9 \\
124 \\
194\end{array}$ & $\begin{array}{l}0.001 \\
0.001 \\
0.001 \\
0.001 \\
0.001\end{array}$ \\
\hline
\end{tabular}




\begin{tabular}{|c|c|c|c|c|c|}
\hline $\begin{array}{l}\text { Characteristic } \\
\text { (Analysis) }\end{array}$ & & $\begin{array}{c}1996 \\
\text { W-26 S }\end{array}$ & $\begin{array}{c}2000 \\
\mathrm{~W}-26 \mathrm{~S}\end{array}$ & $\begin{array}{r}2001 \\
\mathrm{~W}-26 \mathrm{~S} \\
\end{array}$ & IDL' \\
\hline \multicolumn{6}{|c|}{ Plutonium isotopics by TIMS $( \pm 1 \%)$} \\
\hline $\begin{array}{l}{ }^{238} \mathrm{Pu} \\
{ }^{239} \mathrm{Pu} \\
{ }^{240} \mathrm{Pu} \\
{ }^{241} \mathrm{Pu} \\
{ }^{242} \mathrm{Pu} \\
{ }^{244} \mathrm{Pu}\end{array}$ & $\begin{array}{l}(\text { atom \%) } \\
(\text { atom \%) } \\
(\text { atom \%) } \\
(\text { atom \%) } \\
(\text { atom \%) } \\
(\text { atom \%) }\end{array}$ & $\begin{array}{r}1.23 \\
\mathbf{8 2 . 2 7} \\
15.11 \\
\mathbf{0 . 5 7} \\
\mathbf{0 . 8 1} \\
<0.01\end{array}$ & & & - \\
\hline $\begin{array}{l}\frac{\mathrm{Pu} \text { activity }}{{ }^{238} \mathrm{Pu}} \\
{ }^{239} \mathrm{Pu} \\
{ }^{240} \mathrm{Pu} \\
{ }^{241} \mathrm{Pu} \\
{ }^{242} \mathrm{Pu} \\
{ }^{244} \mathrm{Pu} \\
\left({ }^{239} \mathrm{Pu}\right) \\
{ }^{232} \mathrm{Th} /{ }^{239} \mathrm{Pu}\end{array}$ & $\begin{array}{l}(\mathrm{Bq} / \mathrm{g}) \\
(\mathrm{Bq} / \mathrm{g}) \\
(\mathrm{Bq} / \mathrm{g}) \\
(\mathrm{Bq} / \mathrm{g}) \\
(\mathrm{Bq} / \mathrm{g}) \\
(\mathrm{Bq} / \mathrm{g}) \\
(\mathrm{ng} / \mathrm{g})\end{array}$ & $\begin{array}{c}\mathbf{5 4 0 0} \\
1300 \\
890 \\
\mathbf{1 5 0 0 0} \\
\mathbf{0 . 8} \\
<0.1 \\
\\
\mathbf{7 0 0} \\
\mathbf{5 7 3 0}\end{array}$ & - & 765 & \\
\hline
\end{tabular}


Tahle 5 Analytical חata for. Sludge in .TankeWk-27

\begin{tabular}{|c|c|c|c|c|c|c|}
\hline $\begin{array}{l}\text { characteristic } \\
\text { Analysis) }\end{array}$ & " & $\begin{array}{c}1996 \\
W-27 \mathrm{~S} \\
\end{array}$ & $\begin{array}{c}2000 \\
W-27 \mathrm{~S} \\
\end{array}$ & $\begin{array}{c}2001 \\
W-27 \mathrm{~S} \\
\end{array}$ & $\begin{array}{c}2001 \\
\mathrm{~W}-27 \mathrm{H} \\
\end{array}$ & IDL' \\
\hline \multicolumn{7}{|c|}{ Physical properties and miscellaneous data } \\
\hline $\begin{array}{l}\text { Request number } \\
\text { Sample number } \\
\text { गH } \\
\text { Water }^{\mathrm{a}} \\
\text { TS }^{\mathrm{b}} \\
\text { TSS }^{\mathrm{c}} \\
\mathrm{TDS}^{\mathrm{d}} \\
\text { Bulk density }_{\mathrm{TC}^{\mathrm{a}}} \\
\text { TIC }^{\mathrm{r}} \\
\text { TOC }^{\mathrm{g}}\end{array}$ & $\begin{array}{l}(\mathrm{mg} / \mathrm{g}) \\
(\mathrm{mg} / \mathrm{g}) \\
(\mathrm{mg} / \mathrm{g}) \\
(\mathrm{mg} / \mathrm{g}) \\
(\mathrm{g} / \mathrm{mL}) \\
(\mathrm{mg} / \mathrm{Kg}) \\
(\mathrm{mg} / \mathrm{Kg}) \\
\mathrm{m}\end{array}$ & $\begin{array}{c}7749 F \\
960904-248 \\
12.3 \\
54.9 \\
451 \\
\\
\\
1.44 \\
12400 \\
10000 \\
2400\end{array}$ & $\begin{array}{c}10224 \\
000509-004 \\
10.0 \\
\\
68.9 \\
311 \\
270 \\
41.4 \\
1.246 \\
10100 \\
<1000 \\
10100\end{array}$ & $\begin{array}{c}12057 \\
010716-013 \\
8.8 \\
72.7 \\
273 \\
\\
\\
1.169 \\
6200 \\
3900 \\
2300\end{array}$ & $\begin{array}{c}12057 \\
010716-014 \\
11.7 \\
58.5 \\
415 \\
\\
\\
1.340 \\
6800 \\
2700 \\
4000\end{array}$ & $\begin{array}{l}15 \\
15 \\
15\end{array}$ \\
\hline \multicolumn{7}{|c|}{ RCRA Metals $( \pm 10 \%)$} \\
\hline $\begin{array}{ll}\mathbf{A g}^{\mathrm{h}} & (\mathbf{1 0 0}) \\
\mathrm{As} & (100) \\
\mathrm{Ba} & (2000) \\
\mathrm{Cd} & (20) \\
\mathrm{Cr} & (100) \\
\mathrm{Hg} & (4) \\
\mathrm{Ni} & (1000) \\
\mathrm{Pb} & (100) \\
\mathrm{Se} & (20) \\
\mathrm{T} 1 & (18)\end{array}$ & $\begin{array}{l}(\mathrm{mg} / \mathrm{Kg}) \\
(\mathrm{mg} / \mathrm{Kg}) \\
(\mathrm{mg} / \mathrm{Kg}) \\
(\mathrm{mg} / \mathrm{Kg}) \\
(\mathrm{mg} / \mathrm{Kg}) \\
(\mathrm{mg} / \mathrm{Kg}) \\
(\mathrm{mg} / \mathrm{Kg}) \\
(\mathrm{mg} / \mathrm{Kg}) \\
(\mathrm{mg} / \mathrm{Kg}) \\
(\mathrm{mg} / \mathrm{Kg})\end{array}$ & $\begin{array}{cc}< & 1.8 \\
< & 1.4 \\
41.8 \\
14.8 \\
55.3 \\
29.0 \\
48.9 \\
& 157 \\
<\quad 1.4 \\
<\quad 1.4\end{array}$ & $\begin{array}{c}<\mathbf{1} \\
64.2 \\
<16 \\
132 \\
196 \\
84.0 \\
317 \\
<1 \\
<1\end{array}$ & $\begin{array}{c}<1.6 \\
94.6 \\
19.3 \\
267 \\
118 \\
73.9 \\
804 \\
<0.7 \\
<4\end{array}$ & $\begin{array}{c}<1.4 \\
58.1 \\
24.4 \\
83.0 \\
36.2 \\
37.4 \\
200 \\
<0.7 \\
<3\end{array}$ & $\begin{array}{l}0.006 \\
0.011 \\
0.001 \\
0.111 \\
0.008 \\
0.011 \\
0.065 \\
0.341 \\
0.005 \\
0.005\end{array}$ \\
\hline \multicolumn{7}{|c|}{ Process metals $( \pm 10 \%)$} \\
\hline $\begin{array}{l}\mathrm{Al} \\
\mathrm{B} \\
\mathrm{Be} \\
\mathrm{Ca} \\
\mathrm{co} \\
\mathrm{cu} \\
\mathrm{Cs}^{j} \\
\mathrm{Fe} \\
\mathrm{K} \\
\mathrm{Mg} \\
\mathrm{Mn} \\
\mathrm{M} 0 \\
\mathrm{Na} \\
\mathrm{P} \\
\mathrm{Sb} \\
\mathrm{Si} \\
\mathrm{Sr} \\
\mathrm{Th} \\
\mathrm{Ti} \\
\mathrm{U} \\
\mathrm{V} \\
\mathrm{Zn} \\
\mathrm{Zr} \\
\end{array}$ & $\begin{array}{l}\text { (mg/Kg) } \\
(\mathrm{mg} / \mathrm{Kg}) \\
(\mathrm{mg} / \mathrm{Kg}) \\
(\mathrm{mg} / \mathrm{Kg}) \\
(\mathrm{mg} / \mathrm{Kg}) \\
(\mathrm{mg} / \mathrm{Kg}) \\
(\mathrm{mg} / \mathrm{Kg}) \\
(\mathrm{mg} / \mathrm{Kg}) \\
(\mathrm{mg} / \mathrm{Kg}) \\
(\mathrm{mg} / \mathrm{Kg}) \\
(\mathrm{mg} / \mathrm{Kg}) \\
(\mathrm{mg} / \mathrm{Kg}) \\
(\mathrm{mg} / \mathrm{Kg}) \\
(\mathrm{mg} / \mathrm{Kg}) \\
(\mathrm{mg} / \mathrm{Kg}) \\
(\mathrm{mg} / \mathrm{Kg}) \\
(\mathrm{mg} / \mathrm{Kg}) \\
(\mathrm{mg} / \mathrm{Kg}) \\
(\mathrm{mg} / \mathrm{Kg}) \\
(\mathrm{mg} / \mathrm{Kg}) \\
(\mathrm{mg} / \mathrm{Kg}) \\
(\mathrm{mg} / \mathrm{Kg}) \\
(\mathrm{mg} / \mathrm{Kg})\end{array}$ & $\begin{array}{c}2250 \\
5.98 \\
1.10 \\
43700 \\
2.57 \\
14.2 \\
0.892 \\
935 \\
6970 \\
7820 \\
65.4 \\
\\
58200 \\
1000 \\
37.4 \\
3860 \\
107 \\
1290 \\
11700 \\
3.31 \\
360\end{array}$ & $\begin{array}{c}7640 \\
11.9 \\
17.7 \\
26700 \\
16.8 \\
66.3 \\
1.81 \\
3780 \\
4880 \\
4800 \\
387 \\
98.5 \\
30900 \\
\\
<50 \\
6270 \\
163 \\
17400 \\
\\
29500 \\
<1.3 \\
299\end{array}$ & $\begin{array}{c}8580 \\
4.70 \\
8.67 \\
23400 \\
26.2 \\
71.1 \\
<4 \\
3670 \\
4560 \\
3670 \\
314 \\
70.8 \\
23700 \\
\\
<4 \\
7750 \\
87.8 \\
6400 \\
53.1 \\
34300 \\
<1 \\
186 \\
234 \\
\end{array}$ & $\begin{array}{c}5380 \\
3.07 \\
2.32 \\
54100 \\
28.5 \\
22.0 \\
<3 \\
1770 \\
7640 \\
5500 \\
65.7 \\
35.0 \\
52300 \\
\\
<3 \\
13900 \\
122 \\
2250 \\
209 \\
3000 \\
<3.6 \\
460 \\
62.7 \\
\end{array}$ & $\begin{array}{l}0.048 \\
0.024 \\
0.001 \\
0.017 \\
0.060 \\
0.006 \\
0.005 \\
0.233 \\
0.178 \\
0.053 \\
0.001 \\
0.030 \\
0.065 \\
0.13 \\
0.509 \\
0.022 \\
0.001 \\
0.181 \\
0.010 \\
0.105 \\
0.013 \\
0.390 \\
0.008 \\
\end{array}$ \\
\hline
\end{tabular}




\begin{tabular}{|c|c|c|c|c|c|c|}
\hline \multicolumn{2}{|l|}{$\begin{array}{l}\text { Characteristic } \\
\text { (Analysis) }\end{array}$} & $\begin{array}{c}1996 \\
W-27 \mathrm{~S}\end{array}$ & $\begin{array}{c}2000 \\
\mathrm{~W}-27 \mathrm{~S}\end{array}$ & $\begin{array}{c}2001 \\
\mathrm{~W}-27 \mathrm{~S}\end{array}$ & $\begin{array}{c}2001 \\
\mathrm{~W}-27 \mathrm{H}\end{array}$ & IDL', \\
\hline \multicolumn{7}{|c|}{ Semi-quantitative metals by ICP-MS ( $\pm 30-50 \%, *$ indicates data from water leach) } \\
\hline $\begin{array}{l}\mathrm{Au} \text {, gold } \\
\mathrm{Bi} \text {, bismuth } \\
\mathrm{Ce} \text {, cerium } \\
\text { Er, erbium } \\
\mathrm{Eu} \text {, europium } \\
\mathrm{Ga} \text {, gallium } \\
\mathrm{Gd} \text {, gadolinium } \\
\mathrm{Ho} \text {, holmium } \\
\mathrm{I} \text {, iodine } \\
\mathrm{La} \text {, lanthanum } \\
\mathrm{Li} \text {, lithium } \\
\mathrm{Mo} \text {, molybdenum } \\
\mathrm{Nb} \text {, niobium } \\
\mathrm{Rb} \text {, rubidium } \\
\mathrm{Sn} \text {, tin } \\
\mathrm{Ti} \text {, titanium } \\
\mathrm{W} \text {, tungsten } \\
\mathrm{Zr} \text {, zirconium }\end{array}$ & $\begin{array}{l}(\mathrm{mg} / \mathrm{Kg}) \\
(\mathrm{mg} / \mathrm{Kg}) \\
(\mathrm{mg} / \mathrm{Kg}) \\
(\mathrm{mg} / \mathrm{Kg}) \\
(\mathrm{mg} / \mathrm{Kg}) \\
(\mathrm{mg} / \mathrm{Kg}) \\
(\mathrm{mg} / \mathrm{Kg}) \\
(\mathrm{mg} / \mathrm{Kg}) \\
(\mathrm{mg} / \mathrm{Kg}) \\
(\mathrm{mg} / \mathrm{Kg}) \\
(\mathrm{mg} / \mathrm{Kg}) \\
(\mathrm{mg} / \mathrm{Kg}) \\
(\mathrm{mg} / \mathrm{Kg}) \\
(\mathrm{mg} / \mathrm{Kg}) \\
(\mathrm{mg} / \mathrm{Kg}) \\
(\mathrm{mg} / \mathrm{Kg}) \\
(\mathrm{mg} / \mathrm{Kg}) \\
(\mathrm{mg} / \mathrm{Kg})\end{array}$ & $\begin{array}{c}0.62 \\
130 \\
7.2 \\
0.12 \\
0.80 \\
4.2 \\
1.9 \\
1.6 \\
* 6.8 \\
7.3 \\
* 53 \\
* 2.0 \\
0.56 \\
* 1.2 \\
4.0 \\
99 \\
1.3 \\
4.0\end{array}$ & & $\begin{array}{l}218 \\
227\end{array}$ & $\begin{array}{c}8.56 \\
\\
88.8 \\
116\end{array}$ & \\
\hline \multicolumn{7}{|c|}{ Anions by ion chromatography in water wash of sludge $( \pm 10 \%)$} \\
\hline $\begin{array}{l}\text { Inorganic } \\
\text { Bromide } \\
\text { Chloride } \\
\text { Chromate } \\
\text { Fluoride } \\
\text { Nitrate } \\
\text { Nitrite } \\
\text { Phosphate. } \\
\text { Sulphate } \\
\text { Organic } \\
\text { Acetate } \\
\text { Citrate } \\
\text { Fonnate } \\
\text { Oxalate } \\
\text { Phthalate }\end{array}$ & $\begin{array}{c}(\mathrm{mg} / \mathrm{Kg}) \\
(\mathrm{mg} / \mathrm{Kg}) \\
(\mathrm{mg} / \mathrm{Kg}) \\
(\mathrm{mg} / \mathrm{Kg}) \\
(\mathrm{mg} / \mathrm{Kg}) \\
(\mathrm{mg} / \mathrm{Kg}) \\
(\mathrm{mg} / \mathrm{Kg}) \\
\mathrm{m} \\
(\mathrm{mg} / \mathrm{Kg}) \\
(\mathrm{mg} / \mathrm{Kg}) \\
(\mathrm{mg} / \mathrm{Kg}) \\
(\mathrm{mg} / \mathrm{Kg})\end{array}$ & $\begin{array}{c}<50 \\
2280 \\
<20 \\
<50 \\
210000 \\
2283 \\
<20 \\
549 \\
\\
196 \\
<20 \\
200 \\
16.0 \\
<20\end{array}$ & $\begin{array}{c}<41 \\
1190 \\
16.2 \\
246 \\
62500 \\
2600 \\
12.1 \\
943 \\
\\
272 \\
\mathrm{i} 4.1 \\
77.8 \\
228 \\
\dot{<} 4.1\end{array}$ & $\begin{array}{c}140 \\
2450 \\
18.8 \\
20.7 \\
233000 \\
4380 \\
<10 \\
530 \\
\\
448 \\
<5 \\
174 \\
21.2 \\
<5\end{array}$ & $\begin{array}{c}37.8 \\
688 \\
37.5 \\
235 \\
45400 \\
2460 \\
<10 \\
1040 \\
\\
42.3 \\
<4 \\
21 \\
42.3 \\
<4\end{array}$ & $\begin{array}{l}0.05 \\
0.05 \\
0.05 \\
0.05 \\
0.10 \\
0.10 \\
0.20 \\
0.10\end{array}$ \\
\hline
\end{tabular}




\begin{tabular}{|c|c|c|c|c|c|c|}
\hline $\begin{array}{l}\text { characteristi } \\
\text { Analysis) } \\
\end{array}$ & & $\begin{array}{c}1996 \\
\mathrm{~W}-27 \mathrm{~S} \\
\end{array}$ & $\begin{array}{c}2000 \\
\mathrm{~W}-27 \mathrm{~S} \\
\end{array}$ & $\begin{array}{c}2001 \\
\text { W-27 S } \\
\end{array}$ & $\begin{array}{c}2001 \\
\mathrm{~W}-27 \mathrm{H} \\
\end{array}$ & IDL' \\
\hline \multicolumn{7}{|c|}{ Beta/gamma emitters $( \pm 10 \%)$} \\
\hline $\begin{array}{l}\frac{\text { 3ross beta }}{{ }^{19} \mathrm{Ni}} \\
{ }^{33} \mathrm{Ni} \\
{ }^{50} \mathrm{Co} \\
{ }^{20} \mathrm{Sr} / 90 \\
{ }^{19} \mathrm{Tc} \\
{ }^{129} \\
{ }^{134} \mathrm{Cs} \\
{ }^{137} \mathrm{Cs} \\
{ }^{151} \mathrm{Sm} \\
{ }^{152} \mathrm{Eu} \\
{ }^{15}{ }^{154} \mathrm{Eu} \\
{ }^{155} \mathrm{Eu} \\
{ }^{227} \mathrm{Ac} \\
{ }^{241} \mathrm{Pu}\end{array}$ & $\begin{array}{l}(\mathrm{Bq} / \mathrm{g}) \\
(\mathrm{Bq} / \mathrm{g}) \\
(\mathrm{Bq} / \mathrm{g}) \\
(\mathrm{Bq} / \mathrm{g}) \\
(\mathrm{Bq} / \mathrm{g}) \\
(\mathrm{Bq} / \mathrm{g}) \\
(\mathrm{Bq} / \mathrm{g}) \\
(\mathrm{Bq} / \mathrm{g}) \\
(\mathrm{Bq} / \mathrm{g}) \\
(\mathrm{Bq} / \mathrm{g}) \\
(\mathrm{Bq} / \mathrm{g}) \\
(\mathrm{Bq} / \mathrm{g}) \\
(\mathrm{Bq} / \mathrm{g}) \\
(\mathrm{Bq} / \mathrm{g}) \\
(\mathrm{Bq} / \mathrm{g})\end{array}$ & $\begin{array}{r}1.6 \mathrm{e}+06 \\
<2.0 \mathrm{e}+01 \\
1.7 \mathrm{e}+03 \\
1.2 \mathrm{e}+04 \\
4.5 \mathrm{e}+05 \\
8.7 \mathrm{e}+01 \\
\\
<8.1 \mathrm{e}+02 \\
3.9 \mathrm{e}+05 \\
<5.7 \mathrm{e}+02 \\
4.1 \mathrm{e}+04 \\
1.7 \mathrm{e}+04 \\
<2.7 \mathrm{e}+03 \\
<6.2 \mathrm{e}+03 \\
6.5 \mathrm{e}+03\end{array}$ & $\begin{array}{r}1.0 \mathrm{e}+07 \\
9.9 \mathrm{e}+03 \\
4.0 \mathrm{e}+06 \\
\\
6.0 \mathrm{e}+05 \\
2.2 \mathrm{e}+05 \\
<4.2 \mathrm{e}+04 \\
<1.8 \mathrm{e}+04\end{array}$ & $\begin{array}{r}5.3 \mathrm{e}+06 \\
7.1 \mathrm{e}+03 \\
2.1 \mathrm{e}+06 \\
<2.3 \mathrm{e}+02 \\
<1.4 \mathrm{e}+03 \\
6.6 \mathrm{e}+05 \\
\\
9.9 \mathrm{e}+04 \\
2.9 \mathrm{e}+04 \\
5.1 \mathrm{e}+03\end{array}$ & $\begin{array}{r}2.0 \mathrm{e}+06 \\
4.93+03 \\
6.5 \mathrm{e}+05 \\
4.3 \mathrm{e}+02 \\
<1.1 \mathrm{e}+03 \\
4.2 \mathrm{e}+05 \\
\\
2.3 \mathrm{e}+04 \\
7.8 \mathrm{e}+03 \\
<4.0 \mathrm{e}+03\end{array}$ & \\
\hline \multicolumn{7}{|c|}{ Alpha emitters $( \pm 10 \%)$} \\
\hline $\begin{array}{l}\text { Gross alpha } \\
\frac{{ }^{232} \mathrm{Th}}{{ }^{233} \mathrm{U}} \\
{ }^{234} \mathrm{U} \\
{ }^{235} \mathrm{U} \\
{ }^{238} \mathrm{U} \\
{ }^{237} \mathrm{~Np} \\
{ }^{241} \mathrm{Am} \\
{ }^{244} \mathrm{Cm} \\
{ }^{250} \mathrm{Cf} \\
{ }^{252} \mathrm{Cf} \\
\text { Total Pu alpha } \\
{ }^{238} \mathrm{Pu} \\
{ }^{239} \mathrm{Pu} /{ }^{240} \mathrm{Pu} \\
{ }^{242} \mathrm{Pu} \\
\mathrm{TRU} \text { activity } \\
\mathrm{Pu}+\mathrm{Am}(3700)\end{array}$ & $\begin{array}{l}(\mathrm{Bq} / \mathrm{g}) \\
(\mathrm{Bq} / \mathrm{g}) \\
(\mathrm{Bq} / \mathrm{g}) \\
(\mathrm{Bq} / \mathrm{g}) \\
(\mathrm{Bq} / \mathrm{g}) \\
(\mathrm{Bq} / \mathrm{g}) \\
(\mathrm{Bq} / \mathrm{g}) \\
(\mathrm{Bq} / \mathrm{g}) \\
(\mathrm{Bq} / \mathrm{g}) \\
(\mathrm{Bq} / \mathrm{g}) \\
(\mathrm{Bq} / \mathrm{g}) \\
(\mathrm{Bq} / \mathrm{g}) \\
(\mathrm{Bq} / \mathrm{g}) \\
(\mathrm{Bq} / \mathrm{g}) \\
(\mathrm{Bq} / \mathrm{g}) \\
\\
(\mathrm{Bq} / \mathrm{g})\end{array}$ & $\begin{array}{c}26000 \\
5.2 \\
1000 \\
53 \\
2.5 \\
145 \\
12 \\
2800 \\
17000 \\
<100 \\
<100 \\
\\
3400 \\
2200 \\
1200 \\
\end{array}$ & $\begin{array}{r}110000 \\
71 \\
6200 \\
380 \\
11 \\
360 \\
\\
11600 \\
77000\end{array}$ & $\begin{array}{c}62000 \\
26 \\
1360 \\
343 \\
17.2 \\
424 \\
\\
6600 \\
42000\end{array}$ & $\begin{array}{c}24000 \\
9.1 \\
396 \\
<1 \\
0.8 \\
37.2 \\
\\
4000 \\
14500\end{array}$ & \\
\hline \multicolumn{7}{|c|}{ Uranium isotopics by ICP-MS $( \pm 2 \%)$} \\
\hline $\begin{array}{l}{ }^{239} \mathrm{U} \\
{ }^{234} \mathrm{U} \\
{ }^{235} \mathrm{U} \\
{ }^{236} \mathrm{U} \\
{ }^{238} \mathrm{U} \\
{ }^{233} \mathrm{U} / \mathrm{MS} \\
{ }^{235} \mathrm{U} / \mathrm{MS} \\
{ }^{238} \mathrm{U} /{ }^{235} \mathrm{U} F \mathrm{FM}\end{array}$ & $\begin{array}{l}(\text { atom \%) } \\
(\text { atom \%) } \\
(\text { atom \%) } \\
(\text { atom \%) } \\
(\text { atom \%) } \\
\\
(\mathrm{mg} / \mathrm{Kg}) \\
(\mathrm{mg} / \mathrm{Kg}) \\
\text { - }\end{array}$ & $\begin{array}{c}0.025 \\
0.002 \\
0.308 \\
0.006 \\
99.660 \\
\\
2.86 \\
35.6 \\
296\end{array}$ & $\begin{array}{r}0.0598 \\
0.0057 \\
0.5068 \\
<0.0001 \\
99.4276 \\
\\
17.3 \\
148 \\
172\end{array}$ & $\begin{array}{l}0.0113 \\
0.0044 \\
0.6360 \\
0.0001 \\
99.3481 \\
\\
3.79 \\
215 \\
155\end{array}$ & $\begin{array}{c}0.0378 \\
0.0000 \\
0.3454 \\
0.0000 \\
99.6168 \\
\\
1.11 \\
10.2 \\
255\end{array}$ & $\begin{array}{l}0.001 \\
0.001 \\
0.001 \\
0.001 \\
0.001\end{array}$ \\
\hline
\end{tabular}




\begin{tabular}{|c|c|c|c|c|c|c|}
\hline $\begin{array}{l}\text { Characteristic } \\
\text { (Analysis) }\end{array}$ & & $\begin{array}{c}1996 \\
\text { W-27 S }\end{array}$ & $\begin{array}{c}2000 \\
\text { W-27 S }\end{array}$ & $\begin{array}{c}2001 \\
\text { W-27 S }\end{array}$ & $\begin{array}{c}2001 \\
\text { W-27 H } \\
\end{array}$ & IDL' \\
\hline \multicolumn{7}{|c|}{ Plutonium isotopics by TIMS $( \pm \mathbf{1} \%)$} \\
\hline $\begin{array}{l}{ }^{238} \mathrm{Pu} \\
{ }^{239} \mathrm{Pu} \\
{ }^{240} \mathrm{Pu} \\
{ }^{241} \mathrm{Pu} \\
{ }^{242} \mathrm{Pu} \\
{ }^{244} \mathrm{Pu}\end{array}$ & $\begin{array}{l}\text { (atom\%) } \\
\text { (atom\%) } \\
(\text { atom\%) } \\
\text { (atom\%) } \\
(\text { atom\%) } \\
(\text { atom\%) }\end{array}$ & $\begin{array}{r}1.08 \\
84.88 \\
12.64 \\
0.49 \\
0.91 \\
<0.01\end{array}$ & & & & \\
\hline $\begin{array}{l}\frac{\mathrm{Pu} \text { activity }}{{ }^{238} \mathrm{Pu}} \\
{ }^{239} \mathrm{Pu} \\
{ }^{240} \mathrm{Pu} \\
{ }^{241} \mathrm{Pu} \\
{ }^{242} \mathrm{Pu} \\
{ }^{244} \mathrm{Pu} \\
\left.{ }^{239} \mathrm{Pu}\right) \\
{ }^{232} \mathrm{Th} /{ }^{239} \mathrm{Pu}\end{array}$ & $\begin{array}{l}(\mathrm{Bq} / \mathrm{g}) \\
(\mathrm{Bq} / \mathrm{g}) \\
(\mathrm{Bq} / \mathrm{g}) \\
(\mathrm{Bq} / \mathrm{g}) \\
(\mathrm{Bq} / \mathrm{g}) \\
(\mathrm{Bq} / \mathrm{g}) \\
\\
(\mathrm{ng} / \mathrm{g})\end{array}$ & $\begin{array}{c}2400 \\
670 \\
370 \\
6500 \\
0.5 \\
\text { co.1 } \\
\\
350 \\
4390\end{array}$ & & 1336 & 1201 & \\
\hline
\end{tabular}


Table 6 Analytical Data for Sludge in.TanksW-28

\begin{tabular}{|c|c|c|c|c|c|}
\hline \multicolumn{2}{|l|}{$\begin{array}{l}\text { Characteristic } \\
\text { (Analysis) }\end{array}$} & $\begin{array}{c}1996 \\
\mathrm{~W}-28 \mathrm{~s} \\
\end{array}$ & $\begin{array}{r}2000 \\
\mathrm{~W}-28 \mathrm{~s} \\
\end{array}$ & $\begin{array}{c}2001 \\
\mathrm{~W}-28 \mathrm{~S} \\
\end{array}$ & IDL', \\
\hline \multicolumn{6}{|c|}{ Physical properties and miscellaneous data } \\
\hline $\begin{array}{l}\text { Request number } \\
\text { Sample number } \\
\text { pH } \\
\text { Water' }^{\mathbf{a}} \\
\text { TS }^{\mathrm{b}} \\
\text { TSS }^{\mathrm{c}} \\
\text { TDS }^{\mathbf{d}} \\
\text { Bulk density } \\
\text { TC } \\
\text { TIC' } \\
\text { TOC }\end{array}$ & $\begin{array}{l} \\
(\%) \\
(\mathrm{mg} / \mathrm{g}) \\
(\mathrm{mg} / \mathrm{g}) \\
(\mathrm{mg} / \mathrm{g}) \\
(\mathrm{g} / \mathrm{mL}) \\
(\mathrm{mg} / \mathrm{Kg}) \\
(\mathrm{mg} / \mathrm{Kg}) \\
(\mathrm{mg} / \mathrm{Kg})\end{array}$ & $\begin{array}{c}7749 \mathrm{~B} \\
960724-060 \\
12.3 \\
47.3 \\
527 \\
\\
1.37 \\
12800 \\
10200 \\
2600\end{array}$ & $\begin{array}{c}10224 \\
000509-005 \\
11.0 \\
\\
61.7 \\
383 \\
266 \\
117 \\
1.306 \\
8000 \\
<1000 \\
8000\end{array}$ & $\begin{array}{c}12057 \\
010716-015 \\
8.7 \\
65.4 \\
346 \\
\\
\\
1.190 \\
8900 \\
5300 \\
3600\end{array}$ & $\begin{array}{l}15 \\
15 \\
15\end{array}$ \\
\hline \multicolumn{6}{|c|}{ RCRA Metals $( \pm 10 \%)$} \\
\hline $\begin{array}{ll}\mathrm{Ag}^{\mathbf{h}} & (100)^{\mathrm{i}} \\
\mathrm{As} & (100) \\
\mathrm{Ba} & (2000) \\
\mathrm{Cd} & (20) \\
\mathrm{Cr} & (100) \\
\mathrm{Hg} & (4) \\
\mathrm{Ni} & (1000) \\
\mathrm{Pb} & (100) \\
\mathrm{Se} & (20) \\
\mathrm{Tl} & (18)\end{array}$ & $\begin{array}{l}(\mathrm{mg} / \mathrm{Kg}) \\
(\mathrm{mg} / \mathrm{Kg}) \\
(\mathrm{mg} / \mathrm{Kg}) \\
(\mathrm{mg} / \mathrm{Kg}) \\
(\mathrm{mg} / \mathrm{Kg}) \\
(\mathrm{mg} / \mathrm{Kg}) \\
(\mathrm{mg} / \mathrm{Kg}) \\
(\mathrm{mg} / \mathrm{Kg}) \\
(\mathrm{mg} / \mathrm{Kg}) \\
(\mathrm{mg} / \mathrm{Kg})\end{array}$ & $\begin{array}{c}<1.8 \\
<5.0 \\
43.3 \\
\mathbf{2 4 . 9} \\
54.8 \\
6.55 \\
53.6 \\
195 \\
<5.0 \\
5.97\end{array}$ & $\begin{array}{c}<1 \\
64.3 \\
17.7 \\
170 \\
50.6 \\
66.5 \\
396 \\
<1 \\
<1\end{array}$ & $\begin{array}{c}<2.5 \\
76.9 \\
21.9 \\
277 \\
81.7 \\
77.5 \\
758 \\
<1 \\
<6\end{array}$ & $\begin{array}{l}0.006 \\
0.011 \\
0.001 \\
0.111 \\
0.008 \\
0.011 \\
0.065 \\
0.341 \\
0.005 \\
0.005\end{array}$ \\
\hline \multicolumn{6}{|c|}{ Process metals $( \pm 10 \%)$} \\
\hline $\begin{array}{l}\mathrm{Al} \\
\mathrm{B} \\
\mathbf{B e} \\
\mathrm{Ca} \\
\mathrm{co} \\
\mathrm{Cu} \\
\mathrm{Cs}^{\mathbf{j}} \\
\mathrm{Fe} \\
\mathrm{K} \\
\mathrm{Mg} \\
\mathrm{Mn} \\
\mathrm{Mo} \\
\mathrm{Na} \\
\mathbf{P} \\
\mathrm{Sb} \\
\mathrm{Si} \\
\mathrm{Sr}^{\mathbf{m}} \\
\mathrm{Th} \\
\mathrm{Ti} \\
\mathrm{U} \\
\mathrm{V} \\
\mathrm{Zn} \\
\mathrm{Zr}\end{array}$ & $\begin{array}{l}(\mathrm{mg} / \mathrm{Kg}) \\
(\mathrm{mg} / \mathrm{Kg}) \\
(\mathrm{mg} / \mathrm{Kg}) \\
(\mathrm{mg} / \mathrm{Kg}) \\
(\mathrm{mg} / \mathrm{Kg}) \\
(\mathrm{mg} / \mathrm{Kg}) \\
(\mathrm{mg} / \mathrm{Kg}) \\
(\mathrm{mg} / \mathrm{Kg}) \\
(\mathrm{mg} / \mathrm{Kg}) \\
(\mathrm{mg} / \mathrm{Kg}) \\
(\mathrm{mg} / \mathrm{Kg}) \\
(\mathrm{mg} / \mathrm{Kg}) \\
(\mathrm{mg} / \mathrm{Kg}) \\
(\mathrm{mg} / \mathrm{Kg}) \\
(\mathrm{mg} / \mathrm{Kg}) \\
(\mathrm{mg} / \mathrm{Kg}) \\
(\mathrm{mg} / \mathrm{Kg}) \\
(\mathrm{mg} / \mathrm{Kg}) \\
(\mathrm{mg} / \mathrm{Kg}) \\
(\mathrm{mg} / \mathrm{Kg}) \\
(\mathrm{mg} / \mathrm{Kg}) \\
(\mathrm{mg} / \mathrm{Kg}) \\
(\mathrm{mg} / \mathrm{Kg})\end{array}$ & $\begin{array}{c}571 \\
7.33 \\
1.36 \\
45800 \\
3.53 \\
28.0 \\
0.480 \\
599 \\
14600 \\
14500 \\
91.0 \\
\\
61000 \\
907 \\
<18 \\
1080 \\
151 \\
1360 \\
18500 \\
1.54 \\
278\end{array}$ & $\begin{array}{c}3860 \\
7.14 \\
7.62 \\
44600 \\
23.9 \\
57.0 \\
0.401 \\
2180 \\
8860 \\
8760 \\
339 \\
28.0 \\
37200 \\
\\
<50 \\
3000 \\
175 \\
4710 \\
\\
31200 \\
<1.3 \\
368\end{array}$ & $\begin{array}{c}6980 \\
<2.7 \\
7.54 \\
37600 \\
36.3 \\
70.6 \\
<6 \\
3270 \\
7900 \\
7380 \\
379 \\
54.0 \\
32000 \\
\\
<6 \\
5620 \\
139 \\
4820 \\
30.0 \\
41900 \\
<1.5 \\
292 \\
184\end{array}$ & $\begin{array}{l}0.048 \\
0.024 \\
0.001 \\
0.017 \\
0.060 \\
0.006 \\
0.005 \\
0.233 \\
0.178 \\
0.053 \\
0.001 \\
0.030 \\
0.065 \\
0.13 \\
0.509 \\
0.022 \\
0.001 \\
0.181 \\
0.010 \\
0.105 \\
0.013 \\
0.390 \\
0.008\end{array}$ \\
\hline
\end{tabular}




\begin{tabular}{|c|c|c|c|c|c|}
\hline \multicolumn{2}{|l|}{$\begin{array}{l}\text { Characteristic } \\
\text { (Analysis) }\end{array}$} & $\begin{array}{c}1996 \\
W-28 \text { s } \\
\end{array}$ & $\begin{array}{c}2000 \\
W-28 \mathrm{~S} \\
\end{array}$ & $\begin{array}{c}2001 \\
\mathrm{~W}-28 \mathrm{~S} \\
\end{array}$ & IDL' \\
\hline \multicolumn{6}{|c|}{ Semi-quantitative metals by ICP-MS $( \pm 30-50 \%, *$ indicates data from water leach) } \\
\hline $\begin{array}{l}\mathrm{Au} \text {, gold } \\
\mathrm{Bi} \text {, bismuth } \\
\mathrm{Ce} \text {, cerium } \\
\text { Er, erbium } \\
\text { Eu, europium } \\
\mathrm{Ga} \text {, gallium } \\
\mathrm{Gd} \text {, gadolinium } \\
\mathrm{Ho} \text {, holmium } \\
\mathrm{I} \text {, iodine } \\
\mathrm{La} \text {, lanthanum } \\
\mathrm{Li} \text {, lithium } \\
\mathrm{Mo} \text {,molybdenum } \\
\mathrm{Nb} \text {, niobium } \\
\mathrm{Rb} \text {, rubidium } \\
\mathrm{Sn} \text {, tin } \\
\mathrm{Ti} \text {, titanium } \\
\mathrm{W} \text {, tungsten } \\
\mathrm{Zr} \text {, zirconium }\end{array}$ & $\begin{array}{l}(\mathrm{mg} / \mathrm{Kg}) \\
(\mathrm{mg} / \mathrm{Kg}) \\
(\mathrm{mg} / \mathrm{Kg}) \\
(\mathrm{mg} / \mathrm{Kg}) \\
(\mathrm{mg} / \mathrm{Kg}) \\
(\mathrm{mg} / \mathrm{Kg}) \\
(\mathrm{mg} / \mathrm{Kg}) \\
(\mathrm{mg} / \mathrm{Kg}) \\
(\mathrm{mg} / \mathrm{Kg}) \\
(\mathrm{mg} / \mathrm{Kg}) \\
(\mathrm{mg} / \mathrm{Kg}) \\
(\mathrm{mg} / \mathrm{Kg}) \\
(\mathrm{mg} / \mathrm{Kg}) \\
(\mathrm{mg} / \mathrm{Kg}) \\
(\mathrm{mg} / \mathrm{Kg}) \\
(\mathrm{mg} / \mathrm{Kg}) \\
(\mathrm{mg} / \mathrm{Kg}) \\
(\mathrm{mg} / \mathrm{Kg})\end{array}$ & $\begin{aligned} 1.9 \\
12 \\
7.9 \\
0.07 \\
1.5 \\
3.1 \\
6.0 \\
0.97 \\
* 9.1 \\
2.0 \\
* 170 \\
* 2.3 \\
* 0.30 \\
* 1.9 \\
5.9 \\
4.1 \\
1.4 \\
1.8\end{aligned}$ & $\begin{array}{l}- \\
- \\
- \\
- \\
- \\
- \\
- \\
- \\
- \\
- \\
- \\
- \\
- \\
- \\
- \\
- \\
-\end{array}$ & $\begin{array}{c}<6 \\
212 \\
172\end{array}$ & - \\
\hline \multicolumn{6}{|c|}{ Anions by ion chromatography in water wash of sludge $( \pm 10 \%)$} \\
\hline $\begin{array}{l}\text { Inorganic } \\
\text { Bromide } \\
\text { Chloride } \\
\text { Chromate } \\
\text { Fluoride } \\
\text { Nitrate } \\
\text { Nitrite } \\
\text { Phosphate } \\
\text { Sulphate } \\
\text { Organic } \\
\text { Acetate } \\
\text { Citrate } \\
\text { Formate } \\
\text { Oxalate } \\
\text { Phthalate }\end{array}$ & $\begin{array}{l}(\mathrm{mg} / \mathrm{Kg}) \\
(\mathrm{mg} / \mathrm{Kg}) \\
(\mathrm{mg} / \mathrm{Kg}) \\
(\mathrm{mg} / \mathrm{Kg}) \\
(\mathrm{mg} / \mathrm{Kg}) \\
(\mathrm{mg} / \mathrm{Kg}) \\
(\mathrm{mg} / \mathrm{Kg}) \\
(\mathrm{mg} / \mathrm{Kg}) \\
(\mathrm{mg} / \mathrm{Kg}) \\
(\mathrm{mg} / \mathrm{Kg}) \\
(\mathrm{mg} / \mathrm{Kg}) \\
(\mathrm{mg} / \mathrm{Kg}) \\
(\mathrm{mg} / \mathrm{Kg})\end{array}$ & $\begin{array}{c}<50 \\
3460 \\
<20 \\
<50 \\
248000 \\
1120 \\
<20 \\
1773 \\
\\
325 \\
<20 \\
271 \\
19.1 \\
<20\end{array}$ & $\begin{array}{c}<44 \\
1580 \\
10.7 \\
70.6 \\
98800 \\
1750 \\
<8.8 \\
973 \\
\\
349 \\
<4.4 \\
90.1 \\
11.8 \\
<4.4\end{array}$ & $\begin{array}{c}57.3 \\
1210 \\
42.5 \\
277 \\
73500 \\
2400 \\
<10 \\
988 \\
\\
70.1 \\
<5 \\
5.6 \\
119 \\
<5\end{array}$ & $\begin{array}{l}0.05 \\
0.05 \\
0.05 \\
0.05 \\
0.10 \\
0.10 \\
0.20 \\
0.10 \\
\\
0.05 \\
0.50 \\
0.05 \\
0.05 \\
0.05\end{array}$ \\
\hline
\end{tabular}




\begin{tabular}{|c|c|c|c|c|c|}
\hline $\begin{array}{l}\text { Characteristic } \\
\text { (Analysis) }\end{array}$ & & $\begin{array}{c}1996 \\
\mathrm{~W}-28 \mathrm{~S} \\
\end{array}$ & $\begin{array}{c}2000 \\
\mathbf{W}-28 \mathbf{S}\end{array}$ & $\begin{array}{c}2001 \\
\mathbf{W}-28 \mathbf{S}\end{array}$ & IDL' \\
\hline \multicolumn{6}{|c|}{ Beta/gamma emitters $( \pm 10 \%)$} \\
\hline $\begin{array}{l}\text { Gross beta } \\
{ }^{59} \mathrm{Ni} \\
{ }^{53} \mathrm{Ni} \\
{ }^{50} \mathrm{Co} \\
{ }^{30} \mathrm{Sr}{ }^{90} \mathrm{Y} \\
{ }^{39} \mathrm{Tc} \\
{ }^{129} \mathrm{I} \\
{ }^{134} \mathrm{Cs} \\
{ }^{137} \mathrm{Cs} \\
{ }^{151} \mathrm{Sm} \\
{ }^{152} \mathrm{Eu} \\
{ }^{154} \mathrm{Eu} \\
{ }^{155} \mathrm{Eu} \\
{ }^{227} \mathrm{Ac} \\
{ }^{241} \mathrm{Pu}\end{array}$ & $\begin{array}{l}(\mathrm{Bq} / \mathrm{g}) \\
(\mathrm{Bq} / \mathrm{g}) \\
(\mathrm{Bq} / \mathrm{g}) \\
(\mathrm{Bq} / \mathrm{g}) \\
(\mathrm{Bq} / \mathrm{g}) \\
(\mathrm{Bq} / \mathrm{g}) \\
(\mathrm{Bq} / \mathrm{g}) \\
(\mathrm{Bq} / \mathrm{g}) \\
(\mathrm{Bq} / \mathrm{g}) \\
(\mathrm{Bq} / \mathrm{g}) \\
(\mathrm{Bq} / \mathrm{g}) \\
(\mathrm{Bq} / \mathrm{g}) \\
(\mathrm{Bq} / \mathrm{g}) \\
(\mathrm{Bq} / \mathrm{g}) \\
(\mathrm{Bq} / \mathrm{g})\end{array}$ & $\begin{array}{r}3.1 \mathrm{e}+06 \\
<2.5 \mathrm{e}+01 \\
3.3 \mathrm{e}+03 \\
4.2 \mathrm{e}+04 \\
7.0 \mathrm{e}+05 \\
1.2 \mathrm{e}+02 \\
4.1 \mathrm{e}=02 \\
<1.2 \mathrm{e}+03 \\
3.1 \mathrm{e}+05 \\
<5.6 \mathrm{e}+02 \\
8.0 \mathrm{e}+05 \\
2.7 \mathrm{e}+05 \\
7.0 \mathrm{e}+04 \\
<6.7 \mathrm{e}+03 \\
1.2 \mathrm{e}+04\end{array}$ & $\begin{array}{l}5.0 \mathrm{e}+06 \\
1.5 \mathrm{e}+04 \\
1.7 \mathrm{e}+06 \\
- \\
4.8 \mathrm{e}+05 \\
5.2 \mathrm{e}+05 \\
1.4 \mathrm{e}+05 \\
<2.0 \mathrm{e}+04\end{array}$ & $\begin{array}{r}4.8 \mathrm{e}+06 \\
- \\
4.8 \mathrm{e}+03 \\
1.8 \mathrm{e}+06 \\
<3.6 \mathrm{e}+02 \\
<9.3 \mathrm{e}+02 \\
2.7 \mathrm{e}+05 \\
\\
1.2 \mathrm{e}+05 \\
3.2 \mathrm{e}+04 \\
7.8 \mathrm{e}+03\end{array}$ & - \\
\hline \multicolumn{6}{|c|}{ Alpha emitters $( \pm 10 \%)$} \\
\hline 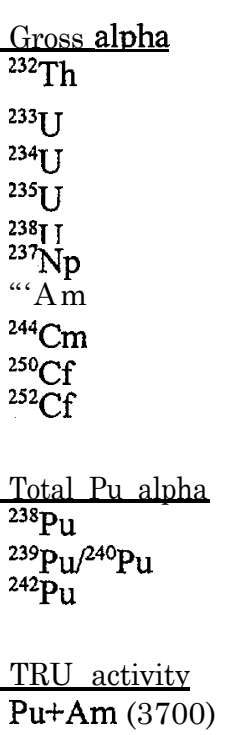 & $\begin{array}{l}(\mathrm{Bq} / \mathrm{g}) \\
(\hat{\mathrm{Bq}} / \mathrm{g}) \\
(\mathrm{Bq} / \mathrm{g}) \\
(\mathrm{Bq} / \mathrm{g}) \\
(\mathrm{Bq} / \mathrm{g}) \\
(\mathrm{Bq} / \mathrm{g}) \\
(\mathrm{Bq} / \mathrm{g}) \\
(\mathrm{Bq} / \mathrm{g}) \\
(\mathrm{Bq} / \mathrm{g}) \\
(\mathrm{Bq} / \mathrm{g}) \\
(\mathrm{Bq} / \mathrm{g}) \\
(\mathrm{Bq} / \mathrm{g})\end{array}$ & $\begin{array}{c}44000 \\
5.5 \\
5200 \\
130 \\
3.8 \\
230 \\
16 \\
4600 \\
25000 \\
<100 \\
<100 \\
\\
4400 \\
2700 \\
1700 \\
- \\
\end{array}$ & $\begin{array}{r}66000 \\
19 \\
4800 \\
360 \\
11 \\
390 \\
\\
7600 \\
43000 \\
\\
\\
11000 \\
6400 \\
4600 \\
\end{array}$ & $\begin{array}{c}58000 \\
20 \\
1680 \\
338 \\
20.3 \\
518 \\
\\
6200 \\
35000 \\
\\
\\
\\
13000 \\
7400 \\
6100 \\
\end{array}$ & - \\
\hline \multicolumn{6}{|c|}{ Uranium isotopics by ICP-MS $( \pm 2 \%)$} \\
\hline $\begin{array}{l}{ }^{233} \mathrm{U} \\
{ }^{234} \mathrm{U} \\
{ }^{235} \mathrm{U} \\
{ }^{236} \mathrm{U} \\
{ }^{238} \mathrm{U} \\
{ }^{233} \mathrm{U} / \mathrm{MS} \\
{ }^{235} \mathrm{U} / \mathrm{MS} \\
{ }^{238} \mathrm{U} /{ }^{235} \mathrm{U} \text { FEM }\end{array}$ & $\begin{array}{l}(\text { atom \%) } \\
(\text { atom \%) } \\
(\text { atom \%) } \\
(\text { atom \%) } \\
(\text { atom \%) } \\
(\mathrm{mg} / \mathrm{Kg}) \\
(\mathrm{mg} / \mathrm{Kg}) \\
-\end{array}$ & $\begin{array}{c}0.081 \\
0.003 \\
0.296 \\
0.007 \\
99.613 \\
\\
14.7 \\
54.1 \\
249\end{array}$ & $\begin{array}{l}0.0441 \\
0.0051 \\
0.4827 \\
0.0033 \\
99.4647 \\
\\
13.5 \\
149 \\
186\end{array}$ & $\begin{array}{l}0.0115 \\
0.0036 \\
0.6141 \\
0.0001 \\
99.3707 \\
\\
4.72 \\
254 \\
160\end{array}$ & $\begin{array}{l}0.001 \\
0.001 \\
0.001 \\
0.001 \\
0.001\end{array}$ \\
\hline
\end{tabular}




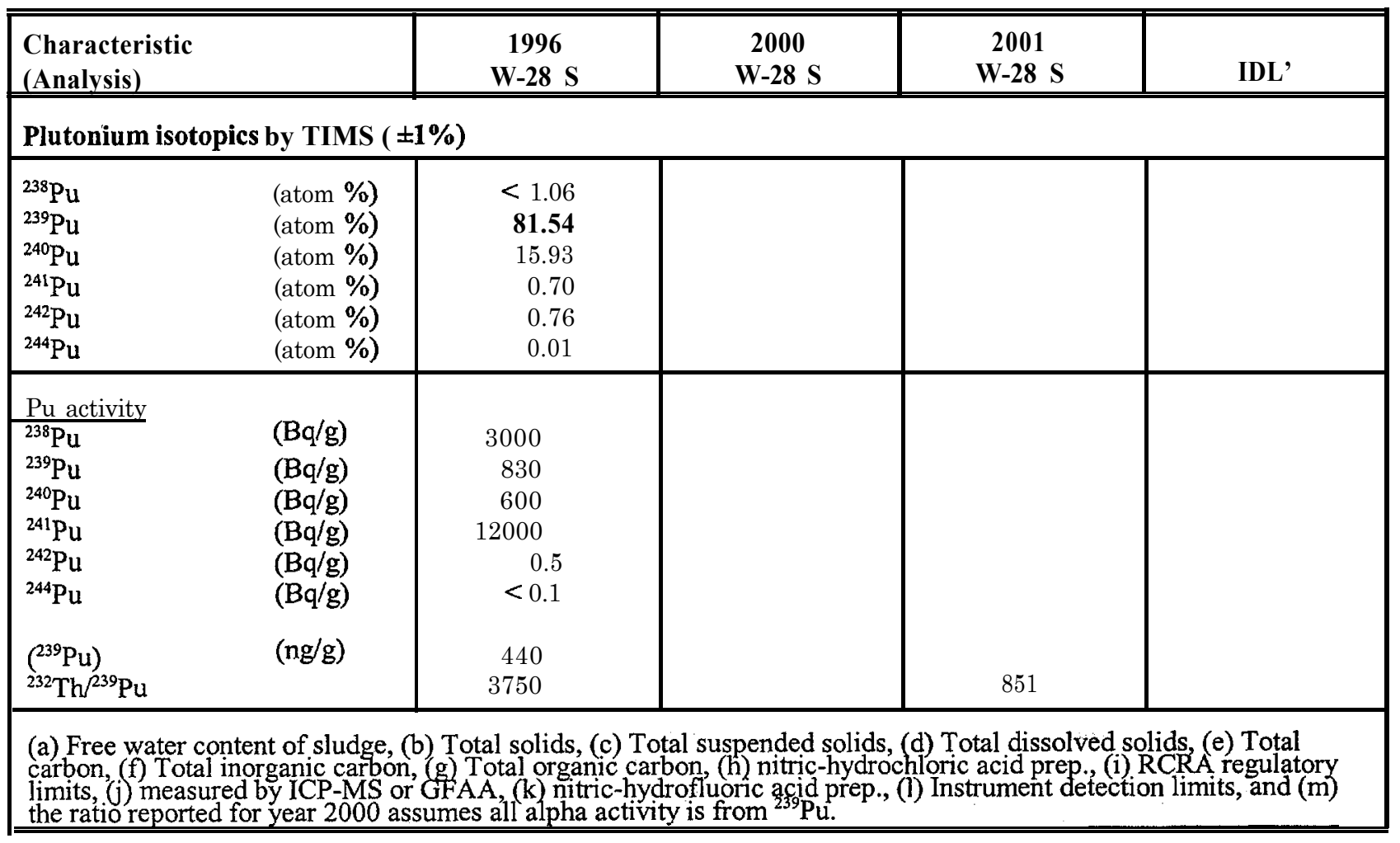


Table 7 Analytical Data for Sludge in Tanks W-29

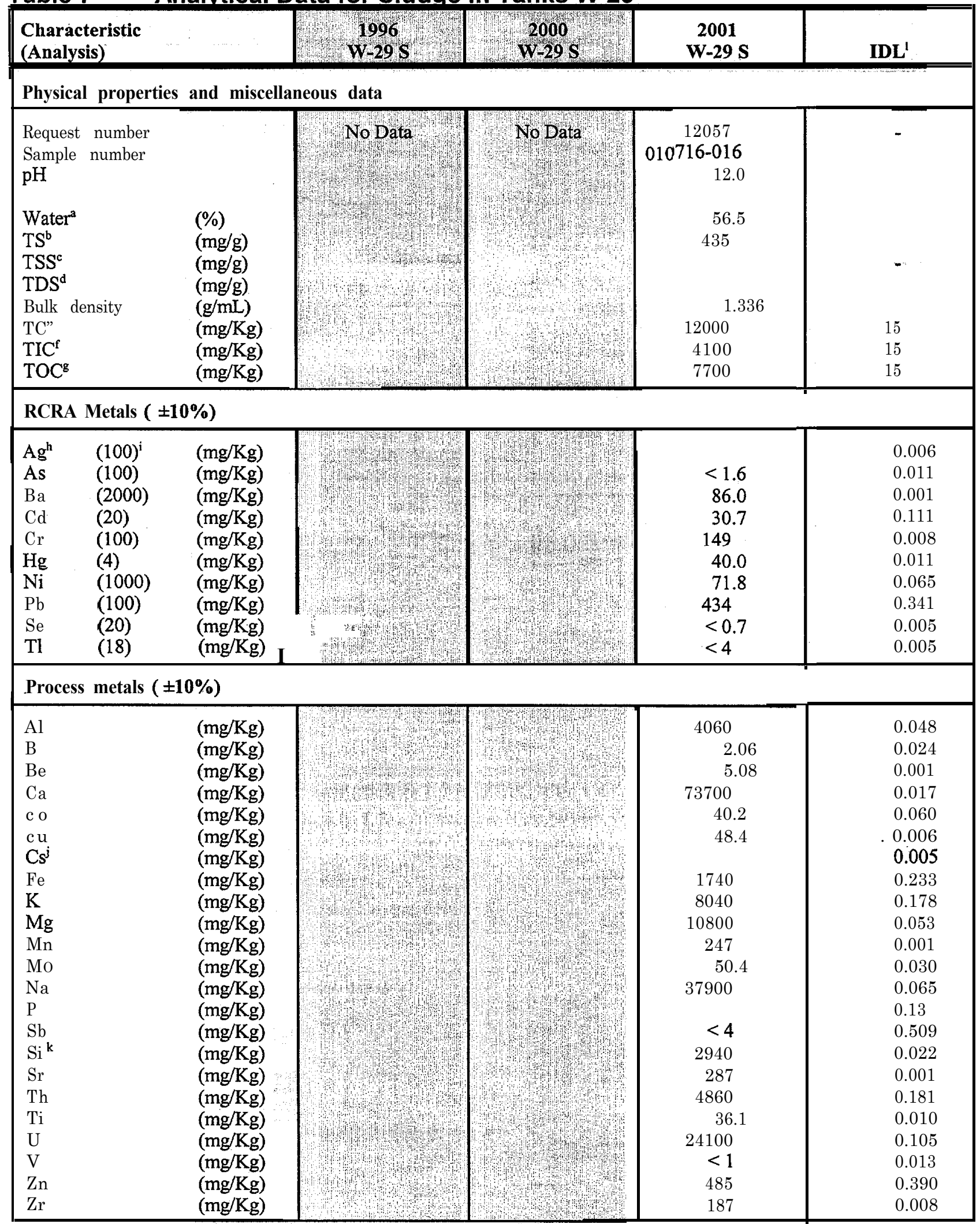




\begin{tabular}{|c|c|c|c|c|c|}
\hline $\begin{array}{l}\text { Characteristic } \\
\text { (Analysis) }\end{array}$ & & $\begin{array}{r}1906 \\
\mathrm{~W}-29 \mathrm{~S}\end{array}$ & $\begin{array}{c}2000 \\
\text { W-29s } \\
\end{array}$ & $\begin{array}{r}2001 \\
\mathrm{w}-29 \mathrm{~s} \\
\end{array}$ & IDL' \\
\hline \multicolumn{6}{|c|}{ Semi-quantitative metals by ICP-MS $( \pm 30-50 \%, *$ indicates data from water leach) } \\
\hline $\begin{array}{l}\mathrm{Au} \text {, gold } \\
\mathrm{Bi} \text {, bismuth } \\
\mathrm{Ce} \text {, cerium } \\
\mathrm{Er} \text {, erbium } \\
\mathrm{Eu} \text {, europium } \\
\mathrm{Ga} \text {, gallium } \\
\mathrm{Gd} \text {, gadolinium } \\
\mathrm{Ho} \text {, holmium } \\
\mathrm{I} \text {, iodine } \\
\mathrm{La} \text {, lanthanum } \\
\mathrm{Li} \text {, lithium } \\
\mathrm{Mo} \text {, molybdenum } \\
\mathrm{Nb} \text {, niobium } \\
\mathrm{Rb} \text {, rubidium } \\
\mathrm{Sn} \text {, tin } \\
\mathrm{Ti} \text {, titanium } \\
\mathrm{W} \text {, tungsten } \\
\mathrm{Zr} \text {, zirconium }\end{array}$ & $\begin{array}{l}(\mathrm{mg} / \mathrm{Kg}) \\
(\mathrm{mg} / \mathrm{Kg}) \\
(\mathrm{mg} / \mathrm{Kg}) \\
(\mathrm{mg} / \mathrm{Kg}) \\
(\mathrm{mg} / \mathrm{Kg}) \\
(\mathrm{mg} / \mathrm{Kg}) \\
(\mathrm{mg} / \mathrm{Kg}) \\
(\mathrm{mg} / \mathrm{Kg}) \\
(\mathrm{mg} / \mathrm{Kg}) \\
(\mathrm{mg} / \mathrm{Kg}) \\
(\mathrm{mg} / \mathrm{Kg}) \\
(\mathrm{mg} / \mathrm{Kg}) \\
(\mathrm{mg} / \mathrm{Kg}) \\
(\mathrm{mg} / \mathrm{Kg}) \\
(\mathrm{mg} / \mathrm{Kg}) \\
(\mathrm{mg} / \mathrm{Kg}) \\
(\mathrm{mg} / \mathrm{Kg}) \\
(\mathrm{mg} / \mathrm{Kg}) \\
\end{array}$ & & & $\begin{array}{l}4.34 \\
60.7 \\
117 \\
<4\end{array}$ & \\
\hline \multicolumn{6}{|c|}{ Anions by ion chromatography in water wash of sludge $( \pm 10 \%)$} \\
\hline $\begin{array}{l}\text { Inorganic } \\
\text { Bromide } \\
\text { Chloride } \\
\text { Chromate } \\
\text { Fluoride } \\
\text { Nitrate } \\
\text { Nitrite } \\
\text { Phosphate } \\
\text { Sulphate } \\
\text { Organic } \\
\text { Acetate } \\
\text { Citrate } \\
\text { Formate } \\
\text { Ox a l a t e } \\
\text { Phthalate }\end{array}$ & $\begin{array}{l}(\mathrm{mg} / \mathrm{Kg}) \\
(\mathrm{mg} / \mathrm{Kg}) \\
(\mathrm{mg} / \mathrm{Kg}) \\
(\mathrm{mg} / \mathrm{Kg}) \\
(\mathrm{mg} / \mathrm{Kg}) \\
(\mathrm{mg} / \mathrm{Kg}) \\
(\mathrm{mg} / \mathrm{Kg}) \\
(\mathrm{mg} / \mathrm{Kg}) \\
\\
(\mathrm{mg} / \mathrm{Kg}) \\
(\mathrm{mg} / \mathrm{Kg}) \\
(\mathrm{mg} / \mathrm{Kg}) \\
(\mathrm{mg} / \mathrm{Kg}) \\
(\mathrm{mg} / \mathrm{Kg})\end{array}$ & $\begin{array}{r}3 \\
\vdots \\
\cdots \\
\cdots\end{array}$ & $\begin{array}{ll} & \\
4\end{array}$ & $\begin{array}{c}120 \\
1380 \\
26 \\
28.9 \\
106000 \\
1920 \\
<10 \\
918 \\
\\
267 \\
<4 \\
99.1 \\
40.6 \\
<4\end{array}$ & $\begin{array}{l}0.05 \\
0.05 \\
0.05 \\
0.05 \\
0.10 \\
0.10 \\
0.20 \\
0.10 \\
\\
0.05 \\
0.50 \\
0.05 \\
0.05 \\
0.05\end{array}$ \\
\hline
\end{tabular}




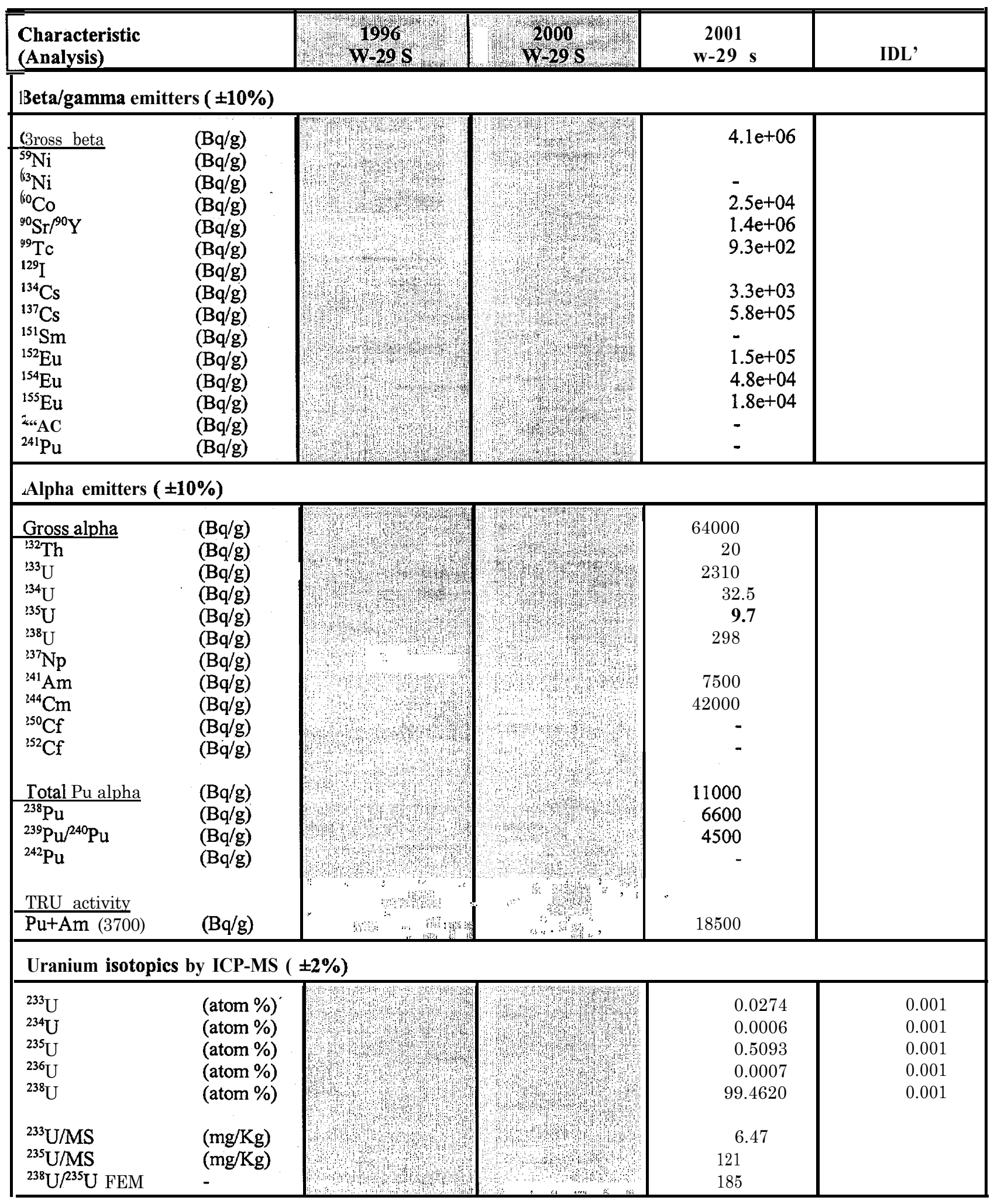




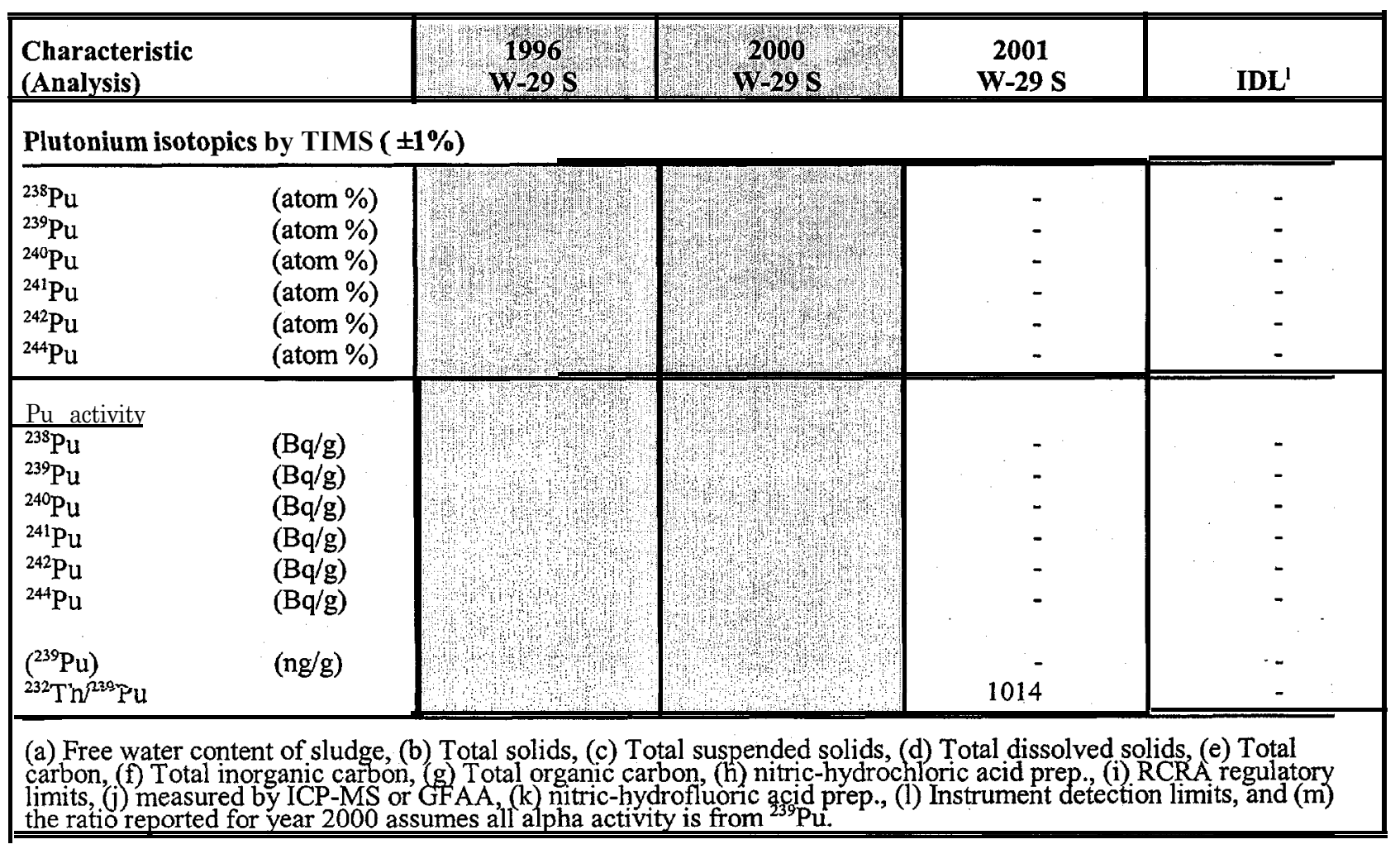


Table 8 Analytical Data for Sludge in Tanks W-30

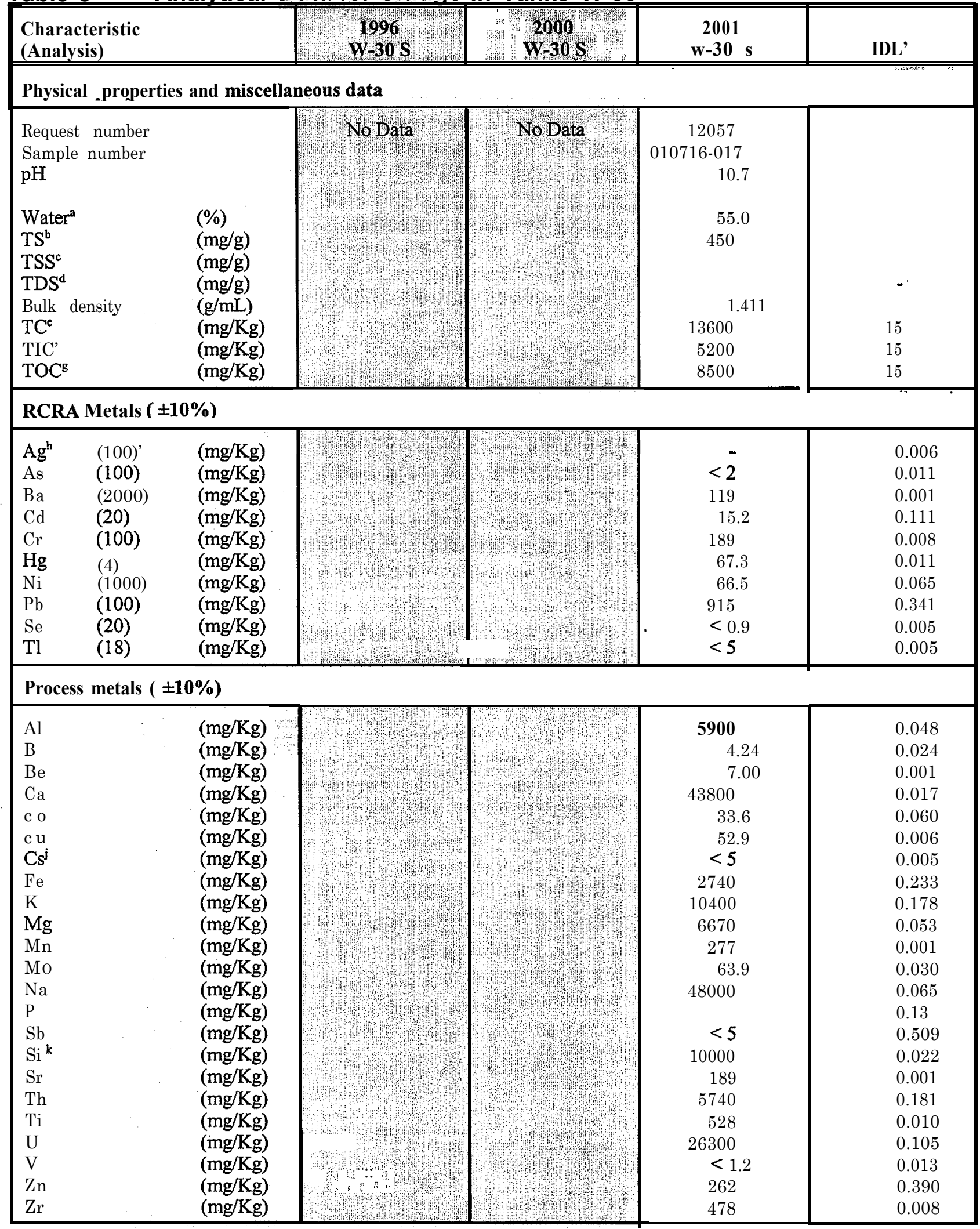




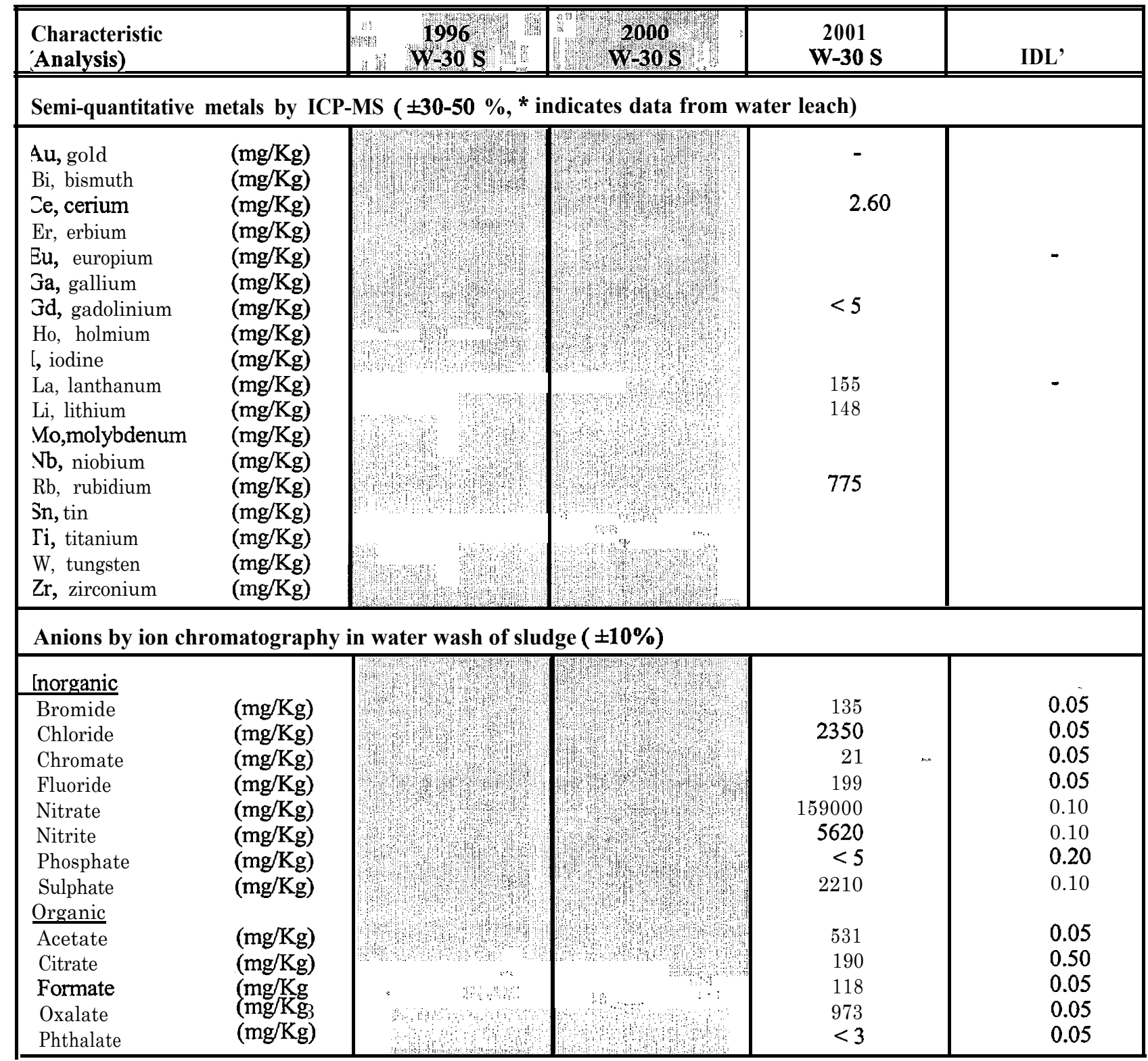




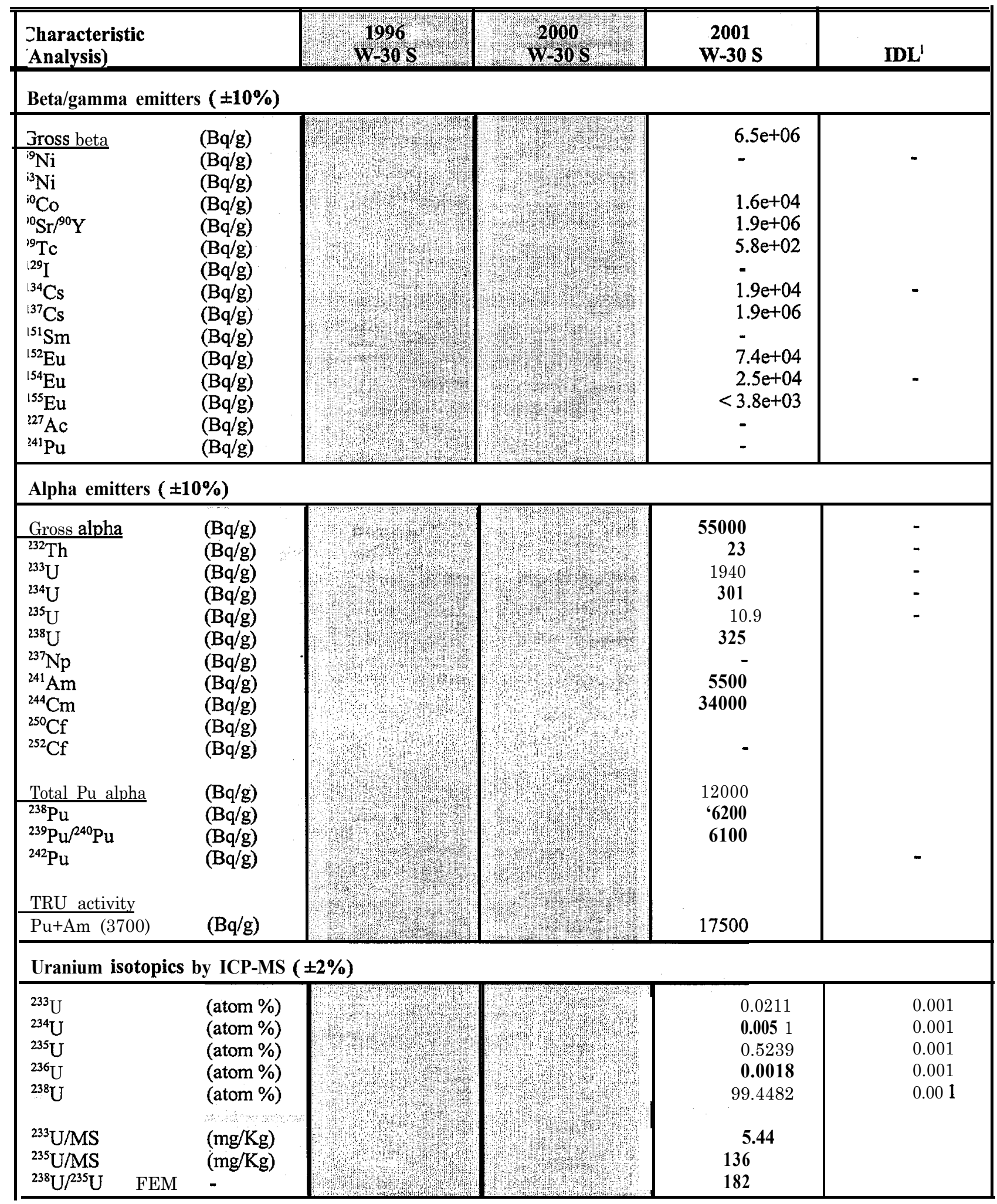




\begin{tabular}{|c|c|c|c|c|c|}
\hline $\begin{array}{l}\text { Characteristic } \\
\text { (Analysis) } \\
\end{array}$ & & $\begin{array}{c}1996 \\
\text {-30 S } \\
\end{array}$ & \begin{tabular}{|l}
2000 \\
$\mathbf{W}-30 \mathrm{~S}$
\end{tabular} & $\begin{array}{c}2001 \\
\mathrm{~W}-30 \mathrm{~S}\end{array}$ & IDL $^{1}$ \\
\hline \multicolumn{6}{|c|}{ Plutonium isotopics by TIMS ( $\pm \mathbf{1} \%)$} \\
\hline $\begin{array}{l}{ }^{238} \mathrm{Pu} \\
{ }^{239} \mathrm{Pu} \\
{ }^{240} \mathrm{Pu} \\
{ }^{241} \mathrm{Pu} \\
{ }^{242} \mathrm{Pu} \\
{ }^{44} \mathrm{Pu}\end{array}$ & $\begin{array}{l}(\text { atom \%) } \\
(\text { atom \%) } \\
(\text { atom \%) } \\
(\text { atom \%) } \\
(\text { atom \%) } \\
(\text { atom \%) }\end{array}$ & 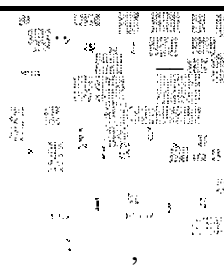 & 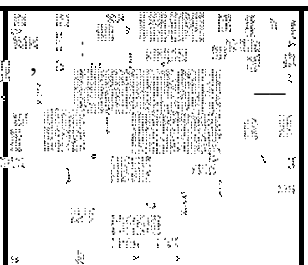 & & \\
\hline $\begin{array}{l}\frac{\mathrm{Pu} \text { activity }}{{ }^{238} \mathrm{Pu}} \\
{ }^{239} \mathrm{Pu} \\
{ }^{240} \mathrm{Pu} \\
{ }^{241} \mathrm{Pu} \\
{ }^{242} \mathrm{Pu} \\
{ }^{244} \mathrm{Pu} \\
\left({ }^{239} \mathrm{Pu}\right) \\
{ }^{232} \mathrm{Th}{ }^{239} \mathrm{Pu}\end{array}$ & $\begin{array}{l}(\mathrm{Bq} / \mathrm{g}) \\
(\mathrm{Bq} / \mathrm{g}) \\
(\mathrm{Bq} / \mathrm{g}) \\
(\mathrm{Bq} / \mathrm{g}) \\
(\mathrm{Bq} / \mathrm{g}) \\
(\mathrm{Bq} / \mathrm{g}) \\
(\mathrm{ng} / \mathrm{g})\end{array}$ & 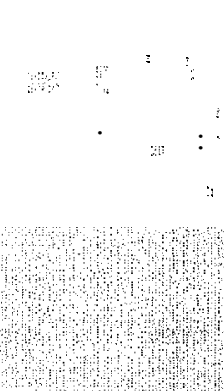 & 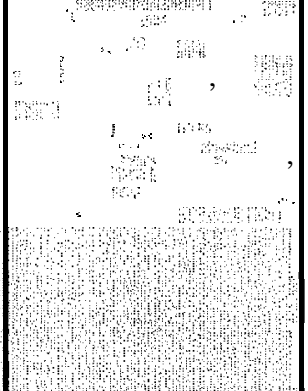 & $\begin{array}{r}- \\
- \\
- \\
- \\
- \\
- \\
-\end{array}$ & \\
\hline
\end{tabular}


Table 9 Analytical-Data for Sludge in Tanks W-31

\begin{tabular}{|c|c|c|c|c|c|c|}
\hline \multicolumn{2}{|l|}{$\begin{array}{l}\text { Characteristic } \\
\text { (Analysis) }\end{array}$} & $\begin{array}{c}1996 \\
w-31 s\end{array}$ & $\begin{array}{c}2000 \\
\mathrm{w}-31 \mathrm{~s}\end{array}$ & $\begin{array}{c}2001 \\
\mathrm{w}-31 \mathrm{~s}\end{array}$ & $\begin{array}{c}2001 \\
\mathrm{~W}-31 \mathrm{H}\end{array}$ & IDL' \\
\hline \multicolumn{7}{|c|}{ Physical properties and miscellaneous data } \\
\hline $\begin{array}{l}\text { Request number } \\
\text { Sample number } \\
\text { pH } \\
\text { Water }^{\mathbf{a}} \\
\text { TS }^{\mathrm{b}} \\
\text { TSS }^{\mathrm{c}} \\
\text { TDS } \\
\text { Bulk density } \\
\text { TC' } \\
\text { TIC' } \\
\text { TOC }\end{array}$ & $\begin{array}{l}(\%) \\
(\mathrm{mg} / \mathrm{g}) \\
(\mathrm{mg} / \mathrm{g}) \\
(\mathrm{mg} / \mathrm{g}) \\
(\mathrm{g} / \mathrm{mL}) \\
(\mathrm{mg} / \mathrm{Kg}) \\
(\mathrm{mg} / \mathrm{Kg}) \\
(\mathrm{mg} / \mathrm{Kg})\end{array}$ & $\begin{array}{c}7749 \mathrm{~A} \\
960717-023 \\
9.9 \\
51.4 \\
486 \\
\\
\\
: 1.44 \\
10200 \\
5300 \\
4900\end{array}$ & $\begin{array}{c}10224 \\
000509-006 \\
9.7 \\
72.5 \\
275 \\
196 \\
79.2 \\
1.210 \\
5690 \\
<1000 \\
5690\end{array}$ & $\begin{array}{c}12057 \\
010716-018 \\
9.3 \\
58.5 \\
415 \\
\\
\\
1.331 \\
14000 \\
9200 \\
4800\end{array}$ & $\begin{array}{c}12057 \\
010716-019 \\
9.3 \\
\\
64.3 \\
357 \\
\\
1.283 \\
11000 \\
8700 \\
2200\end{array}$ & $\begin{array}{l}15 \\
15 \\
15\end{array}$ \\
\hline \multicolumn{7}{|c|}{ RCRA Metals $( \pm 10 \%)$} \\
\hline 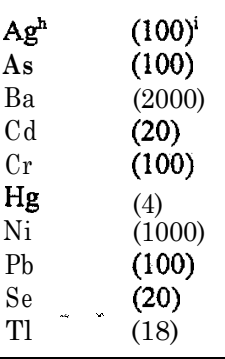 & $\begin{array}{c}(\mathrm{mg} / \mathrm{Kg}) \\
(\mathrm{mg} / \mathrm{Kg}) \\
(\mathrm{mg} / \mathrm{Kg}) \\
(\mathrm{mg} / \mathrm{Kg}) \\
(\mathrm{mg} / \mathrm{Kg}) \\
(\mathrm{mg} / \mathrm{Kg}) \\
(\mathrm{mg} / \mathrm{Kg}) \\
(\mathrm{mg} / \mathrm{Kg}) \\
(\mathrm{mg} / \mathrm{Kg}) \\
(\mathrm{mg} / \mathrm{Kg})\end{array}$ & $\begin{array}{c}<1.9 \\
<5.0 \\
124 \\
\quad 9.03 \\
130 \\
70.7 \\
104 \\
764 \\
<5.0 \\
<5.0\end{array}$ & $\begin{array}{c}<0.9 \\
108 \\
<15 \\
237 \\
65.4 \\
70.3 \\
717 \\
<0.9 \\
<0.9\end{array}$ & $\begin{array}{c}<1.3 \\
100 \\
148 \\
182 \\
70.2 \\
89.1 \\
933 \\
<0.6 \\
<3\end{array}$ & $\begin{array}{c}<2.3 \\
119 \\
<12 \\
325 \\
76.0 \\
74.0 \\
1450 \\
<1 \\
<5\end{array}$ & $\begin{array}{l}0.006 \\
0.011 \\
0.001 \\
0.111 \\
0.008 \\
0.011 \\
0.065 \\
0.341 \\
0.005 \\
0.005\end{array}$ \\
\hline \multicolumn{7}{|c|}{ Process metals $( \pm 10 \%)$} \\
\hline $\begin{array}{l}\mathrm{Al} \\
\mathrm{B} \\
\mathrm{Be} \\
\mathrm{Ca} \\
\mathrm{co} \\
\mathrm{cu} \\
\mathrm{Cs}^{\mathrm{j}} \\
\mathrm{Fe} \\
\mathrm{K} \\
\mathrm{Mg} \\
\mathrm{Mn} \\
\mathrm{Mo} \\
\mathrm{Na} \\
\mathrm{P} \\
\mathrm{Sb} \\
\mathrm{Si} \\
\mathrm{Sr} \\
\mathrm{Th} \\
\mathrm{Ti} \\
\mathrm{U} \\
\mathrm{V} \\
\mathrm{Zn} \\
\mathrm{Zr}\end{array}$ & $\begin{array}{l}(\mathrm{mg} / \mathrm{Kg}) \\
(\mathrm{mg} / \mathrm{Kg}) \\
(\mathrm{mg} / \mathrm{Kg}) \\
(\mathrm{mg} / \mathrm{Kg}) \\
(\mathrm{mg} / \mathrm{Kg}) \\
(\mathrm{mg} / \mathrm{Kg}) \\
(\mathrm{mg} / \mathrm{Kg}) \\
(\mathrm{mg} / \mathrm{Kg}) \\
(\mathrm{mg} / \mathrm{Kg}) \\
(\mathrm{mg} / \mathrm{Kg}) \\
(\mathrm{mg} / \mathrm{Kg}) \\
(\mathrm{mg} / \mathrm{Kg}) \\
(\mathrm{mg} / \mathrm{Kg}) \\
(\mathrm{mg} / \mathrm{Kg}) \\
(\mathrm{mg} / \mathrm{Kg}) \\
(\mathrm{mg} / \mathrm{Kg}) \\
(\mathrm{mg} / \mathrm{Kg}) \\
(\mathrm{mg} / \mathrm{Kg}) \\
(\mathrm{mg} / \mathrm{Kg}) \\
(\mathrm{mg} / \mathrm{Kg}) \\
(\mathrm{mg} / \mathrm{Kg}) \\
(\mathrm{mg} / \mathrm{Kg}) \\
(\mathrm{mg} / \mathrm{Kg})\end{array}$ & $\begin{array}{c}12700 \\
11.6 \\
21.0 \\
24100 \\
4.76 \\
80.2 \\
0.543 \\
2820 \\
8320 \\
2170 \\
247 \\
\\
60600 \\
4240 \\
<19 \\
10200 \\
174 \\
20700 \\
19800 \\
7.18 \\
125\end{array}$ & $\begin{array}{c}7920 \\
7.25 \\
8.91 \\
24700 \\
14.1 \\
64.3 \\
0.395 \\
3420 \\
4570 \\
2090 \\
268 \\
46.7 \\
33700 \\
\\
<45 \\
7930 \\
88.3 \\
8350 \\
\\
38600 \\
<1.1 \\
92.2\end{array}$ & $\begin{array}{c}8700 \\
5.55 \\
15.6 \\
43000 \\
30.3 \\
83.8 \\
<3 \\
2760 \\
7330 \\
4690 \\
270 \\
110 \\
46100 \\
\\
<3 \\
7940 \\
198 \\
13300 \\
22.3 \\
24200 \\
<1 \\
187 \\
437\end{array}$ & $\begin{array}{c}7830 \\
<2.6 \\
6.79 \\
52100 \\
36.1 \\
70.7 \\
<5 \\
3960 \\
5660 \\
2330 \\
262 \\
5 \\
34900 \\
\\
<5 \\
7240 \\
351 \\
5470 \\
26.0 \\
34000 \\
<1.4 \\
129 \\
202\end{array}$ & $\begin{array}{l}0.048 \\
0.024 \\
0.001 \\
0.017 \\
0.060 \\
0.006 \\
0.005 \\
0.233 \\
0.178 \\
0.053 \\
0.001 \\
0.030 \\
0.065 \\
0.13 \\
0.509 \\
0.022 \\
0.001 \\
0.181 \\
0.010 \\
0.105 \\
0.013 \\
0.390 \\
0.008\end{array}$ \\
\hline
\end{tabular}




\begin{tabular}{|c|c|c|c|c|c|c|}
\hline \multicolumn{2}{|l|}{$\begin{array}{l}\text { Characteristic } \\
\text { (Analysis) }\end{array}$} & $\begin{array}{c}1996 \\
\text { W-31 s }\end{array}$ & $\begin{array}{c}2000 \\
\mathrm{~W}-31 \mathrm{~s}\end{array}$ & $\begin{array}{c}2001 \\
\mathrm{~W}-31 \mathrm{~s}\end{array}$ & $\begin{array}{c}2001 \\
\mathrm{~W}-31 \mathrm{H}\end{array}$ & IDL' \\
\hline \multicolumn{7}{|c|}{ Semi-quantitative metals by ICP-MS ( $\pm 30-50 \%, *$ indicates data from water leach) } \\
\hline $\begin{array}{l}\mathrm{Au} \text {, gold } \\
\mathrm{Bi} \text {, bismuth } \\
\mathrm{Ce} \text {, cerium } \\
\mathrm{Er} \text {, erbium } \\
\text { Eu, europium } \\
\mathrm{Ga} \text {, gallium } \\
\mathrm{Gd} \text {, gadolinium } \\
\mathrm{Ho} \text {, holmium } \\
\mathrm{I} \text {, iodine } \\
\mathrm{La} \text {, lanthanum } \\
\mathrm{Li} \text {, lithium } \\
\mathrm{Mo} \text {, molybdenum } \\
\mathrm{Nb} \text {, niobium } \\
\mathrm{Rb} \text {, rubidium } \\
\mathrm{Sn} \text {, tin } \\
\mathrm{Ti} \text {, titanium } \\
\mathrm{W} \text {, tungsten } \\
\mathrm{Zr} \text {, zirconium }\end{array}$ & $\begin{array}{l}(\mathrm{mg} / \mathrm{Kg}) \\
(\mathrm{mg} / \mathrm{Kg}) \\
(\mathrm{mg} / \mathrm{Kg}) \\
(\mathrm{mg} / \mathrm{Kg}) \\
(\mathrm{mg} / \mathrm{Kg}) \\
(\mathrm{mg} / \mathrm{Kg}) \\
(\mathrm{mg} / \mathrm{Kg}) \\
(\mathrm{mg} / \mathrm{Kg}) \\
(\mathrm{mg} / \mathrm{Kg}) \\
(\mathrm{mg} / \mathrm{Kg}) \\
(\mathrm{mg} / \mathrm{Kg}) \\
(\mathrm{mg} / \mathrm{Kg}) \\
(\mathrm{mg} / \mathrm{Kg}) \\
(\mathrm{mg} / \mathrm{Kg}) \\
(\mathrm{mg} / \mathrm{Kg}) \\
(\mathrm{mg} / \mathrm{Kg}) \\
(\mathrm{mg} / \mathrm{Kg}) \\
(\mathrm{mg} / \mathrm{Kg})\end{array}$ & $\begin{array}{c}2.6 \\
1200 \\
20 \\
0.85 \\
0.54 \\
12 \\
0.75 \\
0.22 \\
* 20 \\
54 \\
* 81 \\
* 1.4 \\
2.0 \\
* 1.3 \\
40 \\
34 \\
1.3 \\
51\end{array}$ & & $\begin{array}{l}146 \\
411\end{array}$ & $\begin{array}{l}272 \\
178\end{array}$ & \\
\hline \multicolumn{7}{|c|}{ Anions by ion chromatography in water wash of sludge $( \pm 10 \%)$} \\
\hline $\begin{array}{l}\text { Inorganic } \\
\text { Bromide } \\
\text { Chloride } \\
\text { Chromate } \\
\text { Fluoride } \\
\text { Nitrate } \\
\text { Nitrite } \\
\text { Phosphate } \\
\text { Sulphate } \\
\text { Organic } \\
\text { Acetate } \\
\text { Citrate } \\
\text { Formate } \\
\text { Oxalate } \\
\text { Phthalate }\end{array}$ & $\begin{array}{l}(\mathrm{mg} / \mathrm{Kg}) \\
(\mathrm{mg} / \mathrm{Kg}) \\
(\mathrm{mg} / \mathrm{Kg}) \\
(\mathrm{mg} / \mathrm{Kg}) \\
(\mathrm{mg} / \mathrm{Kg}) \\
(\mathrm{mg} / \mathrm{Kg}) \\
(\mathrm{mg} / \mathrm{Kg}) \\
(\mathrm{mg} / \mathrm{Kg})\end{array}$ & $\begin{array}{c}<50 \\
2570 \\
51.5 \\
125 \\
197000 \\
3470 \\
<50 \\
1090 \\
\\
237 \\
<50 \\
251 \\
89.8 \\
<50\end{array}$ & $\begin{array}{c}<50 \\
817 \\
21.9 \\
604 \\
51900 \\
2680 \\
169 \\
919 \\
\\
<50 \\
<5 \\
<50 \\
107 \\
<5\end{array}$ & $\begin{array}{c}78.9 \\
1610 \\
46.8 \\
370 \\
148000 \\
4710 \\
<10 \\
1240 \\
\\
149 \\
15.1 \\
93.4 \\
261 \\
<5\end{array}$ & $\begin{array}{c}63.8 \\
1220 \\
85.3 \\
920 \\
89800 \\
4140 \\
103 \\
1140 \\
\\
191 \\
<5 \\
15.3 \\
162 \\
<5\end{array}$ & $\begin{array}{l}0.05 \\
0.05 \\
0.05 \\
0.05 \\
0.10 \\
0.10 \\
0.20 \\
0.10 \\
\\
0.05 \\
0.50 \\
0.05 \\
0.05 \\
0.05\end{array}$ \\
\hline
\end{tabular}




\begin{tabular}{|c|c|c|c|c|c|c|}
\hline \multicolumn{2}{|c|}{$\begin{array}{l}\text { Characteristic } \\
\text { (Analysis) }\end{array}$} & $\begin{array}{c}1996 \\
w-31 \mathrm{~s} \\
\end{array}$ & $\begin{array}{c}2000 \\
\mathrm{w}-31 \mathrm{~s}\end{array}$ & $\begin{array}{c}2001 \\
\mathrm{w}-31 \mathrm{~s}\end{array}$ & $\begin{array}{c}2001 \\
\mathrm{~W}-31 \mathrm{H}\end{array}$ & IDL' \\
\hline \multicolumn{7}{|c|}{ Beta/gamma emitters $( \pm 10 \%)$} \\
\hline 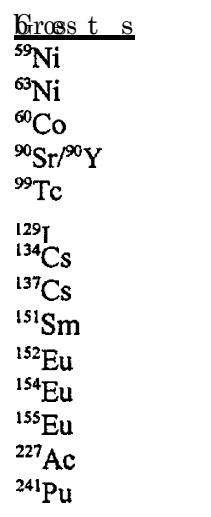 & $\begin{array}{l}(\mathrm{Bq} / \mathrm{g}) \\
(\mathrm{Bq} / \mathrm{g}) \\
(\mathrm{Bq} / \mathrm{g}) \\
(\mathrm{Bq} / \mathrm{g}) \\
(\mathrm{Bq} / \mathrm{g}) \\
(\mathrm{Bq} / \mathrm{g}) \\
(\mathrm{Bq} / \mathrm{g}) \\
(\mathrm{Bq} / \mathrm{g}) \\
(\mathrm{Bq} / \mathrm{g}) \\
(\mathrm{Bq} / \mathrm{g}) \\
(\mathrm{Bq} / \mathrm{g}) \\
(\mathrm{Bq} / \mathrm{g}) \\
(\mathrm{Bq} / \mathrm{g}) \\
(\mathrm{Bq} / \mathrm{g}) \\
(\mathrm{Bq} / \mathrm{g})\end{array}$ & $\begin{array}{r}2.4 \mathrm{e}+07 \\
<3.3 \mathrm{e}+01 \\
4.4 \mathrm{e}+03 \\
2.2 \mathrm{e}+04 \\
1.1 \mathrm{e}+07 \\
1.4 \mathrm{e}+02 \\
4.5 \mathrm{e}-02 \\
2.5 \mathrm{e}+03 \\
4.3 \mathrm{e}+05 \\
<6.0 \mathrm{e}+02 \\
3.0 \mathrm{e}+04 \\
2.0 \mathrm{e}+04 \\
<3.4 \mathrm{e}+03 \\
<5.8 \mathrm{e}+03 \\
2.4 \mathrm{e}+04\end{array}$ & $\begin{array}{r}6.4 \mathrm{e}+06 \\
<6.4 \mathrm{e}+03 \\
2.6 \mathrm{e}+06 \\
\\
\\
5.7 \mathrm{e}+05 \\
<3.0 \mathrm{e}+04 \\
<1.9 \mathrm{e}+04 \\
<1.1 \mathrm{e}+04\end{array}$ & $\begin{array}{l}1.4 \mathrm{e}+07 \\
9.2 \mathrm{e}+03 \\
6.3 \mathrm{e}+06 \\
7.3 \mathrm{e}+02 \\
9.8 \mathrm{e}+02 \\
4.6 \mathrm{e}+05 \\
4.9 \mathrm{e}+04 \\
1.9 \mathrm{e}+04 \\
1.0 \mathrm{e}+04\end{array}$ & $\begin{array}{r}7.6 \mathrm{e}+06 \\
2.3 \mathrm{e}+03 \\
3.2 \mathrm{e}+06 \\
<3.3 \mathrm{e}+02 \\
<1.6 \mathrm{e}+03 \\
6.4 \mathrm{e}+05 \\
1.0 \mathrm{e}+04 \\
<3.6 \mathrm{e}+03 \\
<3.0 \mathrm{e}+03\end{array}$ & - \\
\hline \multicolumn{7}{|c|}{ Alpha emitters $( \pm \mathbf{1 0} \%)$} \\
\hline $\begin{array}{l}\text { Gross alpha } \\
{ }^{232} \mathrm{Th} \\
{ }^{233} \mathrm{U} \\
{ }^{234} \mathrm{U} \\
{ }^{235} \mathrm{U} \\
{ }^{238} \mathrm{U} \\
{ }^{237} \mathrm{~Np} \\
{ }^{241} \mathrm{Am} \\
{ }^{244} \mathrm{Cm} \\
{ }^{250} \mathrm{Cf} \\
{ }^{252} \mathrm{Cf} \\
\text { Total Pualpha } \\
{ }^{238} \mathrm{Pu} \\
{ }^{239} \mathrm{Pu} /{ }^{240} \mathrm{Pu} \\
\text { TRU activity } \\
\text { Pu+Am (3700) }\end{array}$ & $\begin{array}{l}(\mathrm{Bq} / \mathrm{g}) \\
(\mathrm{Bq} / \mathrm{g}) \\
(\mathrm{Bq} / \mathrm{g}) \\
(\mathrm{Bq} / \mathrm{g}) \\
(\mathrm{Bq} / \mathrm{g}) \\
(\mathrm{Bq} / \mathrm{g}) \\
(\mathrm{Bq} / \mathrm{g}) \\
(\mathrm{Bq} / \mathrm{g}) \\
(\mathrm{Bq} / \mathrm{g}) \\
(\mathrm{Bq} / \mathrm{g}) \\
(\mathrm{Bq} / \mathrm{g}) \\
(\mathrm{Bq} / \mathrm{g}) \\
(\mathrm{Bq} / \mathrm{g}) \\
(\mathrm{Bq} / \mathrm{g}) \\
(\mathrm{Bq} / \mathrm{g}) \\
\\
(\mathrm{Bq} / \mathrm{g})\end{array}$ & $\begin{array}{r}160000 \\
84 \\
5200 \\
310 \\
10 \\
244 \\
21 \\
14000 \\
110000 \\
<100 \\
<100 \\
\\
19000 \\
13000 \\
6200\end{array}$ & $\begin{array}{r}43000 \\
34 \\
1970 \\
860 \\
17 \\
480 \\
\\
2900 \\
29000 \\
\\
\\
\\
9300 \\
5200 \\
4100 \\
\end{array}$ & $\begin{array}{r}92000 \\
54 \\
2100 \\
200 \\
12 \\
300 \\
\\
7900 \\
65000 \\
\\
\\
16000 \\
9700 \\
5800 \\
\\
\\
\end{array}$ & $\begin{array}{r}33000 \\
22 \\
12 \\
180 \\
17 \\
420 \\
\\
3200 \\
19000\end{array}$ & \\
\hline \multicolumn{7}{|c|}{ Uranium isotopics by ICP-MS ( $\pm 2 \%)$} \\
\hline $\begin{array}{l}{ }^{233} \mathrm{U} \\
234^{\prime \prime} \\
{ }^{235} \mathrm{U} \\
{ }^{236} \mathrm{U} \\
{ }^{238} \mathrm{U} \\
{ }^{233} \mathrm{U} / \mathrm{MS} \\
{ }^{235} \mathrm{U} / \mathrm{MS} \\
{ }^{238} \mathrm{U} /{ }^{235} \mathrm{U} \text { FEM }\end{array}$ & $\begin{array}{l}(\text { atom \%) } \\
(\text { atom \%) } \\
(\text { atom \%) } \\
(\text { atom \%) } \\
(\text { atom \%) } \\
(\mathrm{mg} / \mathrm{Kg}) \\
(\mathrm{mg} / \mathrm{Kg}) \\
-\end{array}$ & $\begin{array}{r}0.075 \\
0.007 \\
0.750 \\
0.004 \\
99.165 \\
\\
14.5 \\
150 \\
118\end{array}$ & $\begin{aligned} & 0.0146 \\
& 0.0098 \\
& 0.6333 \\
&< 0.0001 \\
& 99.3423 \\
& \\
& \\
& 5.52 \\
& 241 \\
& 154\end{aligned}$ & $\begin{array}{c}0.0250 \\
0.0036 \\
0.6460 \\
0.0000 \\
99.3254 \\
\\
5.92 \\
154 \\
148\end{array}$ & $\begin{array}{l}0.0001 \\
0.0023 \\
0.6471 \\
0.0000 \\
99.3504 \\
\\
\text { co.1 } \\
217 \\
155\end{array}$ & $\begin{array}{l}0.001 \\
0.001 \\
0.001 \\
0.001 \\
0.001\end{array}$ \\
\hline
\end{tabular}




\begin{tabular}{|c|c|c|c|c|c|c|}
\hline \multicolumn{2}{|c|}{$\begin{array}{l}\text { Characteristic } \\
\text { (Analysis) }\end{array}$} & $\begin{array}{c}1996 \\
w-31 \text { s } \\
\end{array}$ & $\begin{array}{r}2000 \\
w-31 \text { s } \\
\end{array}$ & $\begin{array}{c}2001 \\
w-31 \text { s } \\
\end{array}$ & $\begin{array}{c}2001 \\
\text { W-31 H } \\
\end{array}$ & IDL $^{1}$ \\
\hline \multicolumn{7}{|c|}{ Plutonium isotopics by TIMS ( $\pm 1 \%)$} \\
\hline $\begin{array}{l}{ }^{238} \mathrm{Pu} \\
{ }^{239} \mathrm{Pu} \\
{ }^{241} \mathrm{Pu} \\
{ }^{242} \mathrm{Pu} \\
{ }^{24} \mathrm{Pu}\end{array}$ & $\begin{array}{l}(\text { atom\%) } \\
(\text { atom\%) } \\
(\text { atom\%) } \\
(\text { atom\%) } \\
(\text { atom\%) } \\
(\text { atom\%) }\end{array}$ & $\begin{array}{c}<1.16 \\
81.94 \\
14.55 \\
0.34 \\
1.9 \\
0.11\end{array}$ & & - & - & - \\
\hline $\begin{array}{l}\frac{\text { Pu activity }}{{ }^{238} \mathrm{Pu}} \\
{ }^{239} \mathrm{Pu} \\
{ }^{240} \mathrm{Pu} \\
{ }^{241} \mathrm{Pu} \\
{ }^{242} \mathrm{Pu} \\
{ }^{244} \mathrm{Pu} \\
\left.{ }^{233} \mathrm{Pu}\right) \\
{ }^{232} \mathrm{Th} /{ }^{239} \mathrm{Pu}\end{array}$ & $\begin{array}{l}(\mathrm{Bq} / \mathrm{g}) \\
(\mathrm{Bq} / \mathrm{g}) \\
(\mathrm{Bq} / \mathrm{g}) \\
(\mathrm{Bq} / \mathrm{g}) \\
(\mathrm{Bq} / \mathrm{g}) \\
(\mathrm{Bq} / \mathrm{g}) \\
\\
(\mathrm{ng} / \mathrm{g})\end{array}$ & $\begin{array}{c}13000 \\
3400 \\
2200 \\
24000 \\
5.1 \\
<0.1 \\
\\
1820 \\
13800\end{array}$ & - & 1908 & 1460 & \\
\hline \multicolumn{7}{|c|}{ 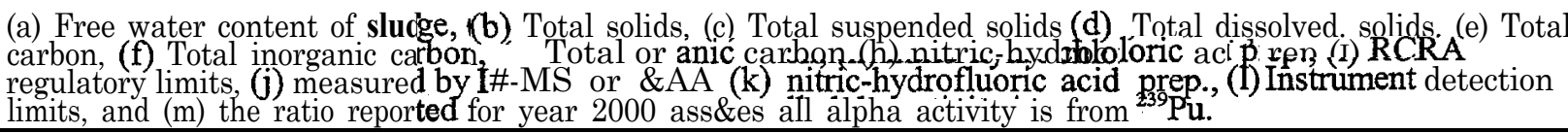 } \\
\hline
\end{tabular}




\subsection{Discussion of MVST Sludqe Characteristics}

Determination of the mass and charge balance for a sludge sample typically has a larger error bar than what is observed with a supematant sample. The assumptions required about the chemical form and the oxidation state of the species present in the sludge are not well'known, and many of the compounds in the sludge are mixed oxides which are not directly measured. The sludge is actually a slurry with a high water content. The interstitial liquid is in close contact with the sludge, and there are many ionic solubility equilibriums. The anion data for the sludge samples discussed in this report are based on the water soluble anions that would be available to a water wash. The water wash does not account for the insoluble hydroxides, carbonates, and mixed oxides present in a sludge sample. The insoluble species do not contribute to the charge balance, and the cation charge is not used in the calculation, as indicated in Table 10. Most of the nitrate reported for the sludge is due to the interstitial liquid. Considering these limitations, the compounds listed in Table 10 were used to estimate the mass and charge balance.

Table 10 Assumption Used for Major Compounds in MVST Sludge

\begin{tabular}{||c|c|c|c||}
\hline \hline Cation & Chemical Form & $\begin{array}{c}\text { Cation } \\
\text { Charge Used }\end{array}$ & $\begin{array}{c}\text { Gravimetric } \\
\text { Factors }\end{array}$ \\
\hline \hline $\mathrm{Al}^{3+}$ & $\mathrm{Al}_{2} \mathrm{O}_{3}$ & $\mathbf{0}$ & 1.890 \\
$\mathrm{Ca}^{2+}$ & $\mathrm{CaCO}_{3}$ & 0 & 2.497 \\
$\mathrm{Fe}^{3+}$ & $\mathrm{Fe}_{2} \mathrm{O}_{3}$ & 0 & 1.430 \\
$\mathrm{~K}^{+}$ & $\mathrm{K}^{+} \mathrm{NO}_{3}$ & +1 & $\mathbf{2 . 5 8 6}$ \\
$\mathrm{Mg}^{2}$ & $\mathrm{Mg}(\mathrm{OH})_{2}$ & $\mathbf{0}$ & $\mathbf{2 . 3 9 9}$ \\
$\mathrm{Mn}^{2+}$ & $\mathrm{Mn}(\mathrm{OH})_{2}$ & $\mathbf{0}$ & 1.619 \\
$\mathrm{Na}^{+}$ & $\mathrm{Na}{ }^{+} \mathrm{NO}_{3}^{-}$ & +1 & 3.697 \\
$\mathrm{Th}^{4+}$ & $\mathrm{Th}(\mathrm{OH})_{4}$ & 0 & 1.293 \\
$\mathrm{UO}_{2}^{2+}$ & $\mathrm{UO}_{2}\left((\mathrm{OH})_{2}-\mathrm{H}_{2} \mathrm{O}\right.$ & $\mathbf{0}$ & 1.353 \\
\hline \hline
\end{tabular}


Table 11 summarizes the mass and charge balance for the MVST tank sludge samples. Considering the limitations of these calculations, the mass balance is within the analytical error $( \pm 20 \%)$ for these sludge samples. The charge balance is more influenced by the chemical form assumptions, and the results have a larger corresponding error range.

Table 11 Summary of Quality Checks for MVST Sludge Data

\begin{tabular}{|c|c|c|c|c|c|c|}
\hline Tank & $\begin{array}{c}\text { Mass } \\
\text { Balance } \\
\left(\mathrm{TS}_{\text {calc. }} / \mathrm{TS}_{\text {meas. }}\right)\end{array}$ & $\begin{array}{l}\text { Charge } \\
\text { Balance } \\
\left(\mathbf{M}^{+} / \mathbf{A}^{-}\right)\end{array}$ & pH & $\begin{array}{c}{ }^{134} \mathrm{Cs}+{ }^{137} \mathrm{Cs} \\
(\%)\end{array}$ & $\begin{array}{c}{ }^{90} \mathrm{Sr}{ }^{90} \mathrm{Y} \\
(\%)\end{array}$ & $\begin{array}{c}\text { Beta } \\
\text { Recovery } \\
(\%)\end{array}$ \\
\hline $\mathrm{W}-24 \mathrm{~S}$ & 0.97 & 0.92 & 10.0 & 18.9 & 64.0 & 95.6 \\
\hline $\mathrm{W}-25 \mathrm{~S}$ & 0.98 & 0.89 & 11.8 & 7.5 & 84.4 & 94.3 \\
\hline $\mathrm{W}-26 \mathrm{~S}$ & 0.98 & 0.83 & 9.4 & 17.9 & 63.3 & 94.9 \\
\hline $\mathrm{W}-27 \mathrm{~S}$ & 0.95 & 0.28 & 8.8 & 15.1 & 82.2 & 97.6 \\
\hline $\mathrm{W}-27 \mathrm{H}$ & 0.99 & 2.66 & 11.7 & 26.8 & 71.1 & 92.6 \\
\hline $\mathrm{W}-28 \mathrm{~S}$ & 0.99 & 1.07 & 8.7 & 7.7 & 88.2 & 86.0 \\
\hline w- $29 \mathrm{~s}$ & 0.98 & 0.97 & 12.0 & 18.1 & 75.0 & 92.5 \\
\hline $\mathrm{w}-30 \mathrm{~s}$ & 0.91 & 0.79 & 10.7 & 36.0 & 61.8 & 95.5 \\
\hline $\mathrm{w}-31 \mathrm{~s}$ & 0.95 & 0.76 & 9.3 & 4.1 & 95.2 & 95.2 \\
\hline W-31 H & 1.03 & 0.86 & 9.3 & 10.4 & 89.4 & 94.6 \\
\hline
\end{tabular}

The beta recovery results are listed in Table 11. As discussed before, the variability for the beta recovery is probably due to the analytical error on the ${ }^{90} \mathrm{Sr}$ measurement. Any measurement error for the ${ }^{90} \mathrm{Sr}$ activity would be doubled when considering the beta recovery calculation.

The distribution, by weight percent, of the major compounds from Table 8 are illustrated in Fig. 1 for each MVST sludge sample. The distribution of the total uranium and thorium concentrations in the MVST sludge samples are shown in Fig. 2, and the change in uranium concentration between sludge sample collected in 1996 and 2000 is shown in Fig. 3. 
Figure 1 Distribution of Major Compounds in the MVST Sludge

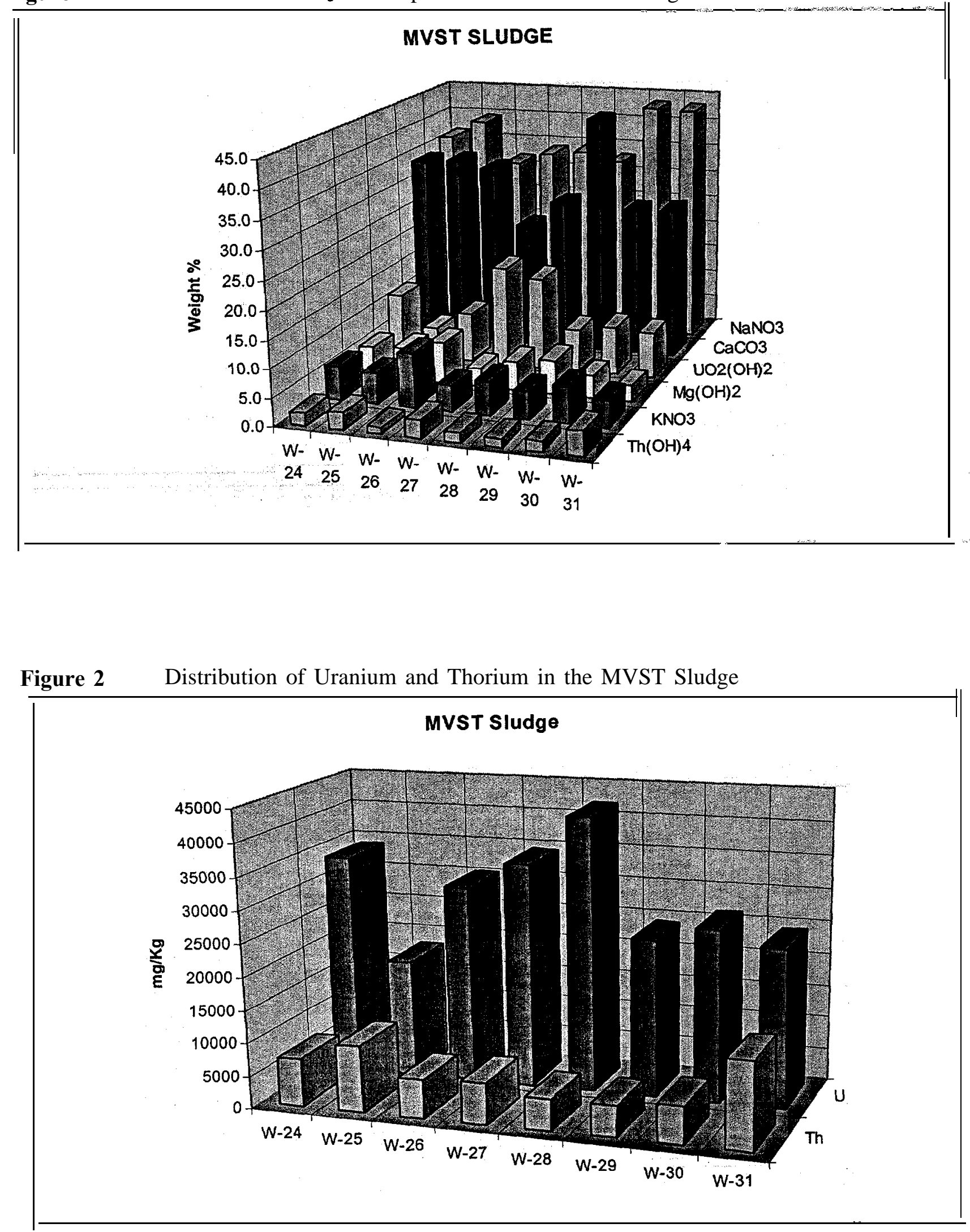


Figure 3 Distribution of Uranium in the MVST Sludge in 1996, 2000, and 2001

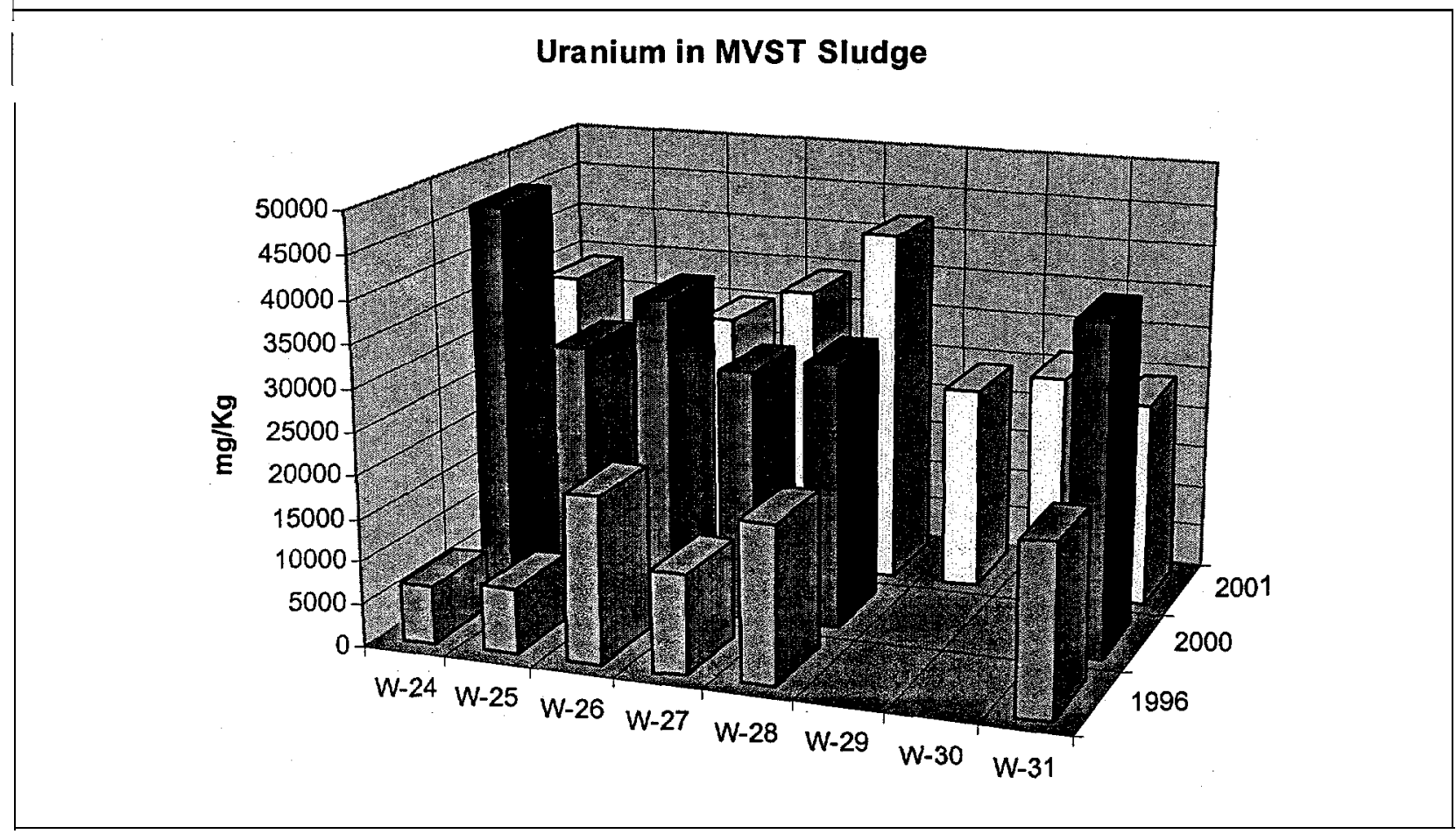

The distribution of the major beta emitters found in the MVST sludge samples are summarized in Table 12. The distributions of the beta activity are shown to be dependent upon the radionuclides present, which is a function of the age of the radioactive waste, and the $\mathrm{pH}$ of the supernatant found over the sludge. Under the typical basic conditions for ORNL waste tanks, the major difference in the beta distribution between the supernatant and the sludge is that the distribution of the longer lived fission products $\left({ }^{90} \mathrm{Sr}\right.$ and $\left.{ }^{137} \mathrm{Cs}\right)$ are reversed due to the differences in solubility. The Group IA metals $\left({ }^{134} \mathrm{Cs}\right.$ and $\left.{ }^{137} \mathrm{Cs}\right)$ and the radionuclides that form anionic species $\left({ }^{99} \mathrm{TcO}_{4}^{-},{ }^{129} \mathrm{~T}^{-}\right.$, and $\left.{ }^{129} \mathrm{IO}_{3}^{-}\right)$are more soluble in the supernatant. The solubility of the Group IIA metals $\left({ }^{90} \mathrm{Sr}\right)$ in the supernatant are a function of both $\mathrm{pH}$ and carbonate concentration. At high $\mathrm{pH}$ most of the other metals, lanthanides, and actinide elements form insoluble hydroxides and mixed oxides, which are found in the sludge. The ${ }^{99} \mathrm{Tc}$ activity is higher in the supernatant than the sludge. The source of most of the ${ }^{99} \mathrm{Tc}$ found in the sludge samples was the interstitial liquid, and not insoluble forms of technetium. The shorter lived radionuclides observed include the europium $\left({ }^{152} \mathrm{Eu},{ }^{154} \mathrm{Eu}\right.$, and $\left.{ }^{155} \mathrm{Eu}\right)$ isotopes and to some extent ${ }^{134} \mathrm{Cs}$. 
Table 12 Distribution of Beta Activity in the MVST Sludge

\begin{tabular}{|c|c|c|c|c|c|c|}
\hline \multirow[b]{2}{*}{ Tank } & \multirow[b]{2}{*}{$\mathbf{p H}$} & \multicolumn{5}{|c|}{ Percent of Total Beta Activity } \\
\hline & & $\begin{array}{c}{ }^{90} \mathrm{Sr} /{ }^{90} \mathrm{Y} \\
(\%)\end{array}$ & $\begin{array}{c}{ }^{134} \mathrm{Cs}+{ }^{137} \mathrm{Cs} \\
(\%)\end{array}$ & $\begin{array}{l}{ }^{60} \mathrm{Co} \\
(\%)\end{array}$ & $\begin{array}{l}{ }^{99} \mathrm{Tc} \\
(\%)\end{array}$ & $\begin{array}{r}{ }^{152,154,155} \mathrm{Eu} \\
(\%)\end{array}$ \\
\hline $\mathrm{W}-24 \mathrm{~s}$ & 10.0 & 64.0 & 18.9 & 0.6 & 0.02 & “15.6 \\
\hline $\mathrm{w}-25 \mathrm{~s}$ & 11.8 & 84.4 & 7.5 & 0.4 & 0.02 & 7.6 \\
\hline $\mathrm{W}-26 \mathrm{~S}$ & 9.4 & 63.3 & 17.9 & 0.8 & 0.03 & 17.9 \\
\hline $\mathrm{W}-27 \mathrm{~S}$ & 8.8 & 82.2 & 15.1 & 0.1 & 0.00 & 2.5 \\
\hline $\mathrm{W}-27 \mathrm{H}$ & 11.7 & 71.1 & 26.8 & 0.3 & 0.02 & 1.6 \\
\hline $\mathrm{W}-28 \mathrm{~S}$ & 8.7 & 88.2 & 7.7 & 0.1 & 0.00 & 3.7 \\
\hline $\mathrm{w}-29 \mathrm{~s}$ & 12.0 & 75.0 & 18.1 & 0.7 & 0.02 & 5.7 \\
\hline $\mathrm{w}-30 \mathrm{~s}$ & 10.7 & 61.8 & 36.0 & 0.3 & 0.01 & 1.5 \\
\hline $\mathrm{w}-31 \mathrm{~s}$ & 9.3 & 95.2 & 4.1 & 0.1 & 0.01 & 0.6 \\
\hline $\mathrm{W}-31 \mathrm{H}$ & 9.3 & 89.4 & 10.4 & 0.0 & 0.00 & 0.1 \\
\hline
\end{tabular}

Table 13 Summary of Actinide Elements in MVST Sludge

\begin{tabular}{|c|c|c|c|c|c|c|c|c|c|c|}
\hline \multirow[b]{2}{*}{ Actinide } & W-24S & W-25S & $W-26 S$ & W-27S & W-27H & W-28S & W-29S & $w-30 s$ & $w-31 s$ & W-31H \\
\hline & \multicolumn{10}{|c|}{ (\% a aftivity) } \\
\hline${ }^{232} \mathrm{Th}$ & 0.02 & 0.04 & 0.03 & 0.04 & 0.04 & 0.03 & 0.03 & 0.04 & 0.06 & 0.07 \\
\hline${ }^{233} U$ & 4.34 & 3.55 & 12.14 & 2.20 & 1.67 & 2.94 & 3.64 & 3.56 & 2.32 & 0.04 \\
\hline${ }^{234} \mathbf{U}$ & 0.12 & 0.09 & 0.32 & 0.55 & 0.00 & 0.60 & 0.05 & 0.56 & 0.22 & 0.56 \\
\hline${ }^{235} \mathrm{U}$ & 0.01 & 0.00 & 0.01 & 0.02 & 0.00 & 0.03 & 0.01 & 0.02 & 0.01 & 0.05 \\
\hline${ }^{238} \mathrm{U}$ & 0.28 & 0.20 & 0.56 & 0.69 & 0.16 & 0.90 & 0.47 & 0.60 & 0.33 & 1.34 \\
\hline${ }^{237} \mathrm{~Np}$ & & & & & & & & & & \\
\hline${ }^{238} \mathrm{Pu}$ & 8.08 & 11.95 & 12.36 & 9.73 & 10.53 & 12.92 & 10.43 & 11.40 & 10.65 & 13.40 \\
\hline${ }^{239+240} \mathrm{Pu}$ & 6.33 & 8.27 & 7.59 & 7.95 & 7.58 & 10.65 & 7.11 & 11.21 & 6.37 & 13.72 \\
\hline${ }^{241} \mathrm{Am}^{\mathrm{a}}$ & 10.10 & 7.90 & 7.44 & 10.70 & 16.85 & 10.82 & 11.85 & 10.11 & 8.67 & 10.21 \\
\hline${ }^{244} \mathrm{Cm}$ & 70.71 & 68.00 & 59.55 & 68.11 & 63.18 & 61.10 & 66.39 & 62.50 & 71.37 & 60.61 \\
\hline $\mathrm{G} \alpha$ & 150000 & 110000 & 68000 & 62000 & 24000 & 58000 & 64000 & 55000 & 92000 & 33000 \\
\hline
\end{tabular}

a The "'Am data is based on subtracting the ${ }^{238} \mathrm{Pu}$ by ICP-MS from the alpha peak measured at $5.15 \mathrm{MeV}\left({ }^{238} \mathrm{Pu}+{ }^{241} \mathrm{Am}\right)$ in the alpha spectrum. 
The distribution of the alpha activity is summarized in Table 13, which includes the percent alpha for each MVST sludge sample. In general, the alpha activity in the MVST system is strongly weighted by the ${ }^{244} \mathrm{Cm}$, which has a high specific activity. The list of actinides in Table 13 required several radiochemical and inorganic analytical measurements to generate the best estimates for each of the alpha activities. The ${ }^{232} \mathrm{Th}$ activity is calculated from the total thorium measured by ICP-AES. The other thorium isotopes $\left({ }^{228} \mathrm{Th},{ }^{229} \mathrm{Th}\right.$, and $\left.{ }^{230} \mathrm{Th}\right)$ are present in the ORNL sludge waste at such low mass, their presence would not effect the ICP-AES measurement. The uranium isotopes are measured by ICP-MS. The atom \% results are converted to weight $\%$, which is used to calculate the concentration of each uranium isotope from the total uranium results obtained by ICP-AES. The activity for each uranium radionuclide is then calculated from the specific activity for each isotope. The plutonium isotopes are first measured by ICP-MS, and the total plutonium alpha activity, measured after a chemical separation, is, used to calculate the activity for each isotope. The ${ }^{244} \mathrm{Cm}$ was measured directly by alpha spectrometry without any chemical separation. The ${ }^{241} \mathrm{Am}$ activity is determined by subtracting the ${ }^{238} \mathrm{Pu}$ activity from the sum of the ${ }^{238} \mathrm{Pu}+{ }^{241} \mathrm{Am}$ measured by alpha spectrometry. Both ${ }^{238} \mathrm{Pu}$ and ${ }^{241} \mathrm{Am}$ have an alpha energy of about $5.50 \mathrm{MeV}$ and can not be resolved by alpha spectrometry. There was no chemical separation of the plutonium and americium for this project because of cost concerns.

\subsection{RCRA Characteristics for the MVST Svstem}

The RCRA regulatory limits are listed in Table 14, which also includes the limits for the EPA Toxicity Characteristic Leaching Protocol (TCLP) extract and the functional total metal limits for a solid or sludge waste. The total metal limits are a factor of twenty times higher than the TCLP extraction limits and are based on the 1:20 dilution used for the TCLP extraction procedure. 
Table 14 Summary of RCRA Regulatory Limits

\begin{tabular}{|ll|c|c|}
\hline Metals & & $\begin{array}{c}\text { TCLP Extract } \\
\text { and Liquids } \\
\text { (mg/L) }\end{array}$ & $\begin{array}{c}\text { Solid/Sludge } \\
\text { Total Metal } \\
\text { (mg/Kg) }\end{array}$ \\
\hline Silver & $(\mathrm{Ag})$ & 5 & 100 \\
Arsenic & $(\mathrm{As})$ & 5 & 100 \\
Barium & $(\mathrm{Ba})$ & 100 & 2000 \\
Cadmium & $(\mathrm{Cd})$ & 1 & 20 \\
Chromium & $(\mathrm{Cr})$ & 5 & 100 \\
Mercury & $(\mathrm{Hg})$ & 0.2 & 4 \\
Nickel & $(\mathrm{Ni})$ & 50 & 1000 \\
Lead & $(\mathrm{Pb})$ & 5 & 100 \\
Selenium & $(\mathrm{Se})$ & 1 & 20 \\
Thallium & $(\mathrm{Tl})$ & 0.9 & 18 \\
\hline \hline
\end{tabular}

If the RCRA metal concentrations are found to be below the total metal limits, the solid waste can not fail the TCLP leach test. If the RCRA metal concentrations exceed the total metal limits, the TCLP leach test must be done to determine if the solid waste is hazardous. For solid samples, the TCLP leach test is only valid for the final waste form ready for disposal. The total metal concentration data can be used as acceptable process knowledge if the final waste form only results in a dilution of the RCRA metal concentrations. Examples of waste forms that result in a dilution of a solid waste includes grouting ( 2 fold dilution) and vitrification ( 3 fold dilution). If the total metal limit is exceeded after stabilizing the waste, the TCLP leach test would be required for only the metals that had the potential to exceed the regulatory limits.

All of the MVST tank sludge samples exceed the total metal limits for lead and mercury, and two tanks are over or near the limit for chromium.. Most of the ORNL radioactive waste sludge samples, characterized to date, have exceeded the total metal limits for these three RCRA metals. Based on past experience, it is expected that solidification of the ORNL MVST sludge would fix these RCRA metals such that the final waste form would pass the TCLP leach test. 


\subsection{TRU Classifications for LLLW Svstem}

The DOE definition for Transuranic (TRU) Waste includes the following conditions,

- $\quad$ TRU activity $\geq 3700 \mathrm{~Bq} / \mathrm{g}(100 \mathrm{nCi} / \mathrm{g})$,

- TRU isotopes must be alpha emitting actinide with $\mathrm{Z}>92$ (uranium),

- $\quad$ TRU isotopes must have a half life $\geq 20$ years.

This definition excludes all thorium and uranium isotopes. The short lived actinide ${ }^{244} \mathrm{Cm}\left(\mathrm{t}_{1 / 2}=18.1\right.$ years), which is common to ORNL waste, falls outside the TRU definition. Also, the plutonium isotope, ${ }^{241} \mathrm{Pu}$, would be excluded from calculation of the TRU activity because it is a pure beta emitter. The primary actinide elements common to ORNL waste, that are present at sufficient levels to meet the TRU definition, include ${ }^{238} \mathrm{Pu},{ }^{239} \mathrm{Pu},{ }^{240} \mathrm{Pu}$, and ${ }^{241} \mathrm{Am}$. There is some current work the Radiochemical Engineering Development Center (Mark-42 fuel assembly processing) that could generate enough ${ }^{243} \mathrm{Am}$ to make a significant contribution to TRU alpha content of the waste. The remaining actinide elements present in ORNL waste are generally not available at high enough activity, and/or do not have a long enough half-life to meet the TRU definition.

None of the MVST supematant samples which were analyzed in previous sampling campaigns had enough alpha activity to be considered as TRU waste. All of the MVST sludge that has been characterized to date has been classified as TRU waste based on only the plutonium and americium activity. The alpha activity reported is based on wet weight, if adjusted for dry weight the activity would almost double. The MVST sludge samples contained enough plutonium and americium activity to easily satisfy the WIPP waste acceptance criteria** for transuranic waste. Based on the TRU activity, any dilution of the sludge that would result from a solidification process such as grouting or vitrification would most likely not effect the TRU classification.

\subsection{Distribution of Fissile Material in LLLW Svstem}

As discussed in section 3.5, the ORNL LLLW waste acceptance criteria (WAC) requires the fissile isotopes of uranium and plutonium to be diluted with ${ }^{238} \mathrm{U}$ and ${ }^{232} \mathrm{Th}$, respectively. A summary of the dilution ratios for fissile material in the sludge samples is provided in Table 15. All the dilution ratios for the MVST sludge samples exceed the required dilution factors for the fissile isotopes of 
uranium and plutonium. All the dilution ratios listed in Table 15 are based onequations discussed in section 3.5 of this report.

Table '15 Summary of Denature Ratios for MVST Sludge

\begin{tabular}{|c|c|c|c|}
\hline Tank & $\begin{array}{c}{ }^{238} \mathbf{U} /^{235} \mathbf{U} f_{35} \\
(>110)^{\mathrm{a}}\end{array}$ & $\begin{array}{l}{ }^{238} U /{ }^{233} U \\
(>200)^{b}\end{array}$ & 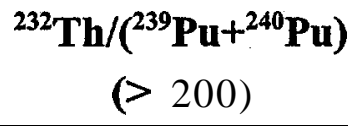 \\
\hline W-24 & 206 & 1017 & $\begin{array}{lll}7 & 6 & 1\end{array}$ \\
\hline$w-25$ & 203 & 906 & 1054 \\
\hline W-26 & 194 & 718 & 765 \\
\hline W-27 S & 155 & 2730 & 1336 \\
\hline $\mathrm{W}-27-\mathrm{H}$ & 255 & 1680 & 1201 \\
\hline W-28 & 160 & 2900 & 851 \\
\hline$w-29$ & 185 & 1640 & 1014 \\
\hline$w-30$ & 182 & 2060 & 1098 \\
\hline $\mathrm{w}-31 \mathrm{~s}$ & 148 & 1190 & 1908 \\
\hline $\mathrm{W}-31 \mathrm{H}$ & 155 & 284000 & 1460 \\
\hline
\end{tabular}

${ }^{\mathrm{a}}$ Calculation based on equation 1 in Section 3.5. ${ }^{\mathrm{b}}$ Calculation based' on equationı 2 in Section 3.5., ${ }^{\mathrm{c}}$ Conservativ estimate of ${ }^{239} \mathrm{Pu}$ dilution since ${ }^{240} \mathrm{Pu}$ is included.

The dilution ratios listed in Tables 15 are based on the ratio of weight $\%$, not the ratio of atom $\%$ given in the data tables. There is a small difference between atom $\%$, reported for the uranium and plutonium, and weight $\%$, which is needed for many calculations performed with the analytical data. To convert from atom $\%$ to weight $\%$, we used the following equation,

$$
W_{i}=\frac{a_{i} M_{i}}{\sum_{i}^{n} a_{i} M_{i}} \times 100 \%
$$

$$
\text { where, } \quad \begin{array}{ll}
\mathrm{W}_{\mathrm{i}} & =\text { weight } \%, \\
\mathrm{M}_{\mathbf{i}} & =\text { nuclidic mass } \\
\mathrm{a}_{\mathrm{i}} & =\text { atom } \% .
\end{array}
$$

An example of this calculation is provided in Table 16, which shows there is not much difference between the atom $\%$ and the weight $\%$. 
Table 16 Example of Converting Atom \% to Weight \% for W-31 Sludge

\begin{tabular}{||c|c|c|r|r||}
\hline Isotope & $\begin{array}{c}\text { Nuclidic mass } \\
\mathbf{( g / m o l})\end{array}$ & atom \% & $\left.\mathbf{( a}_{\mathbf{i}} \mathbf{M}_{\mathbf{i}}\right)$ & weight \% \\
\hline${ }^{233} \mathrm{U}$ & $\mathbf{2 3 3 . 0 3 9 6 2 9}$ & 0.056 & 13.0502 & 0.0548 \\
${ }^{234} \mathrm{U}$ & 234.040947 & 0.004 & 0.9362 & 0.0039 \\
${ }^{235} \mathrm{U}$ & $\mathbf{2 3 5 . 0 4 3 9 2 4}$ & 0.621 & 145.9623 & 0.6132 \\
${ }^{236} \mathrm{U}$ & 236.045563 & 0.002 & 0.4721 & 0.0020 \\
${ }^{238} \mathrm{U}$ & 238.050785 & 99.316 & 23642.2518 & 99.3260 \\
& & & & \\
Total & & 99.999 & 23802.6726 & 99.9999 \\
\hline \hline
\end{tabular}

The distribution of plutonium isotopes by alpha activity are illustrated in Fig. 4 for each of the MVST samples. One should note that the ${ }^{238} \mathrm{Pu}$ dominates the alpha activity and the ${ }^{239} \mathrm{Pu}$ is the major isotope by weight or concentration.

Figure 4 Distribution of Plutonium Alpha Activity in the MVST Sludge

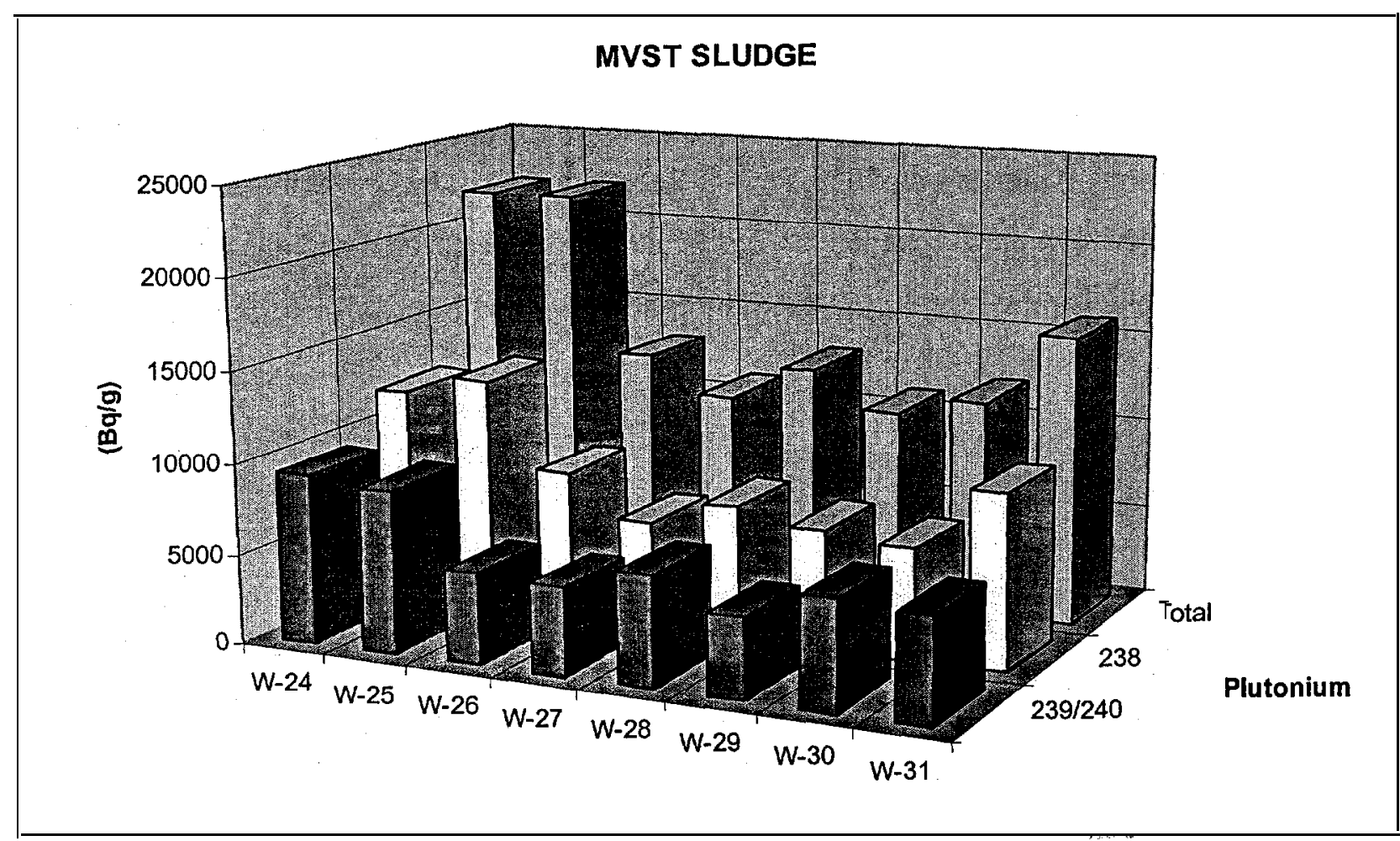




\section{REFERENCES}

1. M. B. Sears, J. L. Botts, R. N. Ceo, J.J. Ferrada, W. H. Griest, J. M. Keller, and R. L. Schenley, Sampling and Analysis of Radioactive Liquid Wastes and Sludges in the Melton Valley and Evaporator Facility Storage Tanks at ORNL, ORNL/TM-11652, September 1990.

2. U. S. Environmental Protection Agency, Test Methods for Evaluating Solid Waste, SW-846, 3rd ed, Office of Solid Waste and Emergency Response, Washington, D.C., November 1986; Update I, July 1992; and Final Update II, September 1994.

3. J. M. Giaquinto, A. M. Essling, and J. M. Keller, Comparison of SW-846 Method 3051 and SW-846 Method 7471A for the Preparation of Solid Waste Samples for Mercury Determination, ORNL/TM-13236, July 1996.

4. Radioactive Materials Analysis Laboratory Quality Assurance Planfoi the Characterization of Radioactive Waste, QAP-X-96-CASDRML-001, Rev. 3, August 2000.

5. J. M. Keller, J. M. Giaquinto, A. M. Meeks, Characterization of the MVST Waste Tanb Located at ORNL, ORNL/TM-13357, December 1996.

6. J. M. Keller and J. M. Giaquinto, Characterization of the MVST Waste Tanks Located at ORNL After Transfer of Sludge BVEST, GAAT and OHF Tanks, ORNL/TM-2000/323, January 2001.

7. Waste Acceptance Criteria for the Waste Isolation Pilot Plant, WIPP-DOE-069, Rev. 5.0, April 1996. 


\section{APPENDIX A}

The following diagram graphically illustrates where sludge samples were collected from each MVST tank during the 2000 sampling campaign. Several of the other ORNL waste tanks systems were transferred to the MVST system prior to the sludge sample being collected. This diagram also provides an estimate of which MVST tanks collected these transfers. There were only minor changes in the contents of these tanks prior to this sampling campaign. The sludge samples for this campaign consisted of a full core from the top to the bottom of the sludge layers. 
A-2 

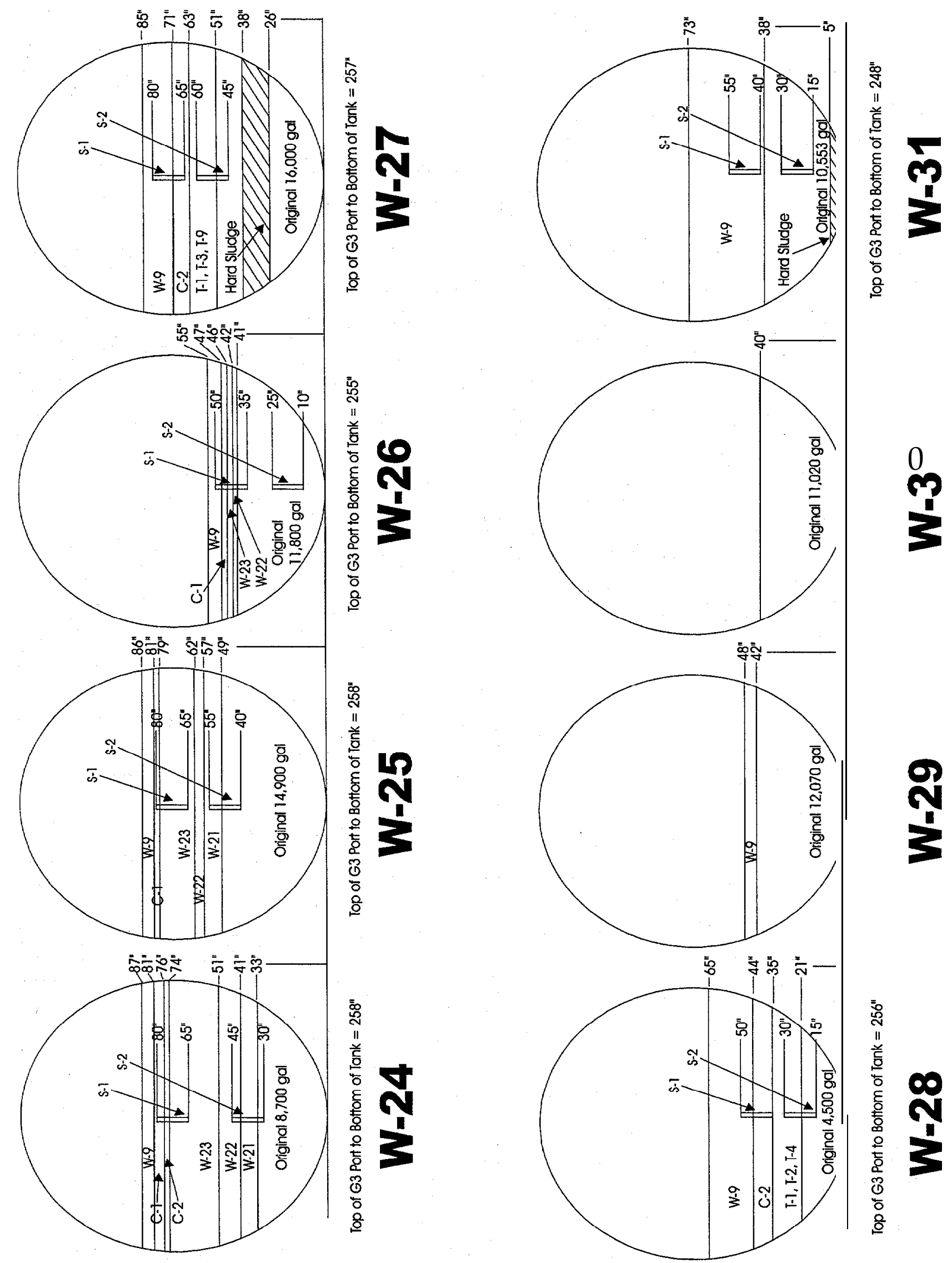

8 


\title{
A P PENDIX B
}

\author{
Radioactive Materials Analytical Laboratory
}

QC Acceptance Criteria for Radioactive Liquid/Solid Waste Samples

\begin{tabular}{|c|c|c|c|c|}
\hline Analysis & $\begin{array}{l}\text { Method (s) } \\
\text { CASD-AM- }\end{array}$ & $\begin{array}{c}\text { Quality Control } \\
\text { Check } \\
\text { (per batch) }\end{array}$ & $\begin{array}{c}\text { SW-846 } \\
\text { Acceptance } \\
\text { Criteria } \\
(\% \mathbf{D}, \% \mathrm{R}, \mathrm{RPD}) \\
\end{array}$ & $\begin{array}{c}\text { RMAL } \\
\text { Acceptance } \\
\text { Criteria } \\
\text { (\%D, \%R, RPD) } \\
\end{array}$ \\
\hline $\begin{array}{l}\text { Metals by ICP-AES } \\
\text { (inductively } \\
\text { coupled plasma } \\
\text { atomic emission } \\
\text { spectroscopy) }\end{array}$ & SW846-6010A & $\begin{array}{l}\text { high standard } \\
\text { calibration verifications (ICV \& CCV) } \\
\text { calibration blank \& checks (ICB \& CCB) } \\
\text { method blank (sample prep) } \\
\text { matrix spike } \\
\text { matrix spike duplicate or sample duplicate } \\
\text { laboratory control sample (sample prep) } \\
\text { serial dilution (if interference suspected) } \\
\text { post digestion spike }\end{array}$ & $\begin{array}{c} \pm 5 \% \mathrm{D} \\
\pm 10 \% \mathrm{D} \\
<3 \times \mathrm{IDL} \\
<3 \times \text { IDL } \\
\pm 20 \% \mathrm{D} \\
\pm 20 \mathrm{RPD} \\
\text { none specified } \\
\pm 10 \% \mathrm{R} \\
\pm 20 \% \mathrm{D}\end{array}$ & $\begin{array}{c} \pm 5 \% \mathrm{D} \\
\pm 10 \% \mathrm{D} \\
<3 \times \mathrm{IDL} \\
<3 \times \mathrm{IDL} \\
\pm 25 \% \mathrm{D} \text { (liq.), } \pm 30 \% \mathrm{D} \text { (solid) } \\
\pm 20 \mathrm{RPD} \text { (liq.), } \pm 30 \mathrm{RPD} \text { (solid) } \\
\pm 20 \% \mathrm{D} \\
\pm 10 \% \mathrm{R} \\
\pm 25 \% \mathrm{D} \text { (liq.), } \pm 30 \% \mathrm{D} \text { (solid) }\end{array}$ \\
\hline $\begin{array}{l}\text { Metals by ICP-MS } \\
\text { (inductively } \\
\text { coupled plasma- } \\
\text { mass spectrometry) }\end{array}$ & SW846-6020 & $\begin{array}{l}\text { calibration verifications (ICV \& CCV) } \\
\text { calibration blank \& blank checks (CCB) }^{\mathrm{b}} \\
\text { method blank (sample prep) } \\
\text { matrix spike } \\
\text { matrix spike duplicate or sample duplicate } \\
\text { laboratory control sample (sample prep) } \\
\text { intemal standard } \\
\text { post digestion spike }\end{array}$ & $\begin{array}{c} \pm 10 \% \mathrm{D} \\
<3 \times \text { IDL } \\
\text { none specified } \\
\text { none specified } \\
\pm 20 \mathrm{RPD} \\
\text { none specified } \\
30-120 \% \mathrm{R} \\
\pm 10 \% \mathrm{D}\end{array}$ & $\begin{array}{c} \pm 10 \% \mathrm{D} \\
<3 \times \mathrm{IDL} \\
<10 \times \mathrm{IDL} \\
\pm 25 \% \mathrm{D} \text { (liq.), } \pm 30 \% \mathrm{D} \text { (solid) } \\
\pm 20 \text { RPD (liq.), } \pm 30 \mathrm{RPD} \text { (solid) } \\
\pm 20 \% \mathrm{D} \\
\pm 30 \% \mathrm{D} \\
\pm 20 \% \mathrm{D}\end{array}$ \\
\hline $\begin{array}{l}\text { Metals by GFAA } \\
\text { (graphite furnace } \\
\text { atomic absorption) }\end{array}$ & SW846-7000A & $\begin{array}{l}\text { high standard } \\
\text { calibration verifications (ICV \& CCV) } \\
\text { method blank (sample prep) } \\
\text { matrix spike } \\
\text { matrix spike duplicate } \\
\text { laboratory control sample (sample prep) } \\
\text { serial dilution (if interference suspected) } \\
\text { post digestion spike }\end{array}$ & $\begin{array}{c}\text { not required } \\
\pm 10 \% \mathrm{D} \text { (ICV), } \pm 20 \% \mathrm{D}(\mathrm{CCV}) \\
\text { none specified } \\
\text { none specified } \\
\text { none specified } \\
\text { none specified } \\
\pm 10 \% \mathrm{R} \\
\pm 15 \% \mathrm{D}\end{array}$ & $\begin{array}{c} \pm 5 \% \mathrm{D} \\
\pm 10 \% \mathrm{D} \text { (ICV), } \pm 20 \% \mathrm{D} \text { (CCV) } \\
<3 \times \mathrm{IDL} \\
\pm 25 \% \mathrm{D} \text { (liq.), } \pm 30 \% \mathrm{D} \text { (solid) } \\
\pm 20 \mathrm{RPD} \text { (liq.), } \pm 30 \mathrm{RPD} \text { (solid) } \\
\pm 25 \% \mathrm{D} \\
\pm 10 \% \mathrm{R} \\
\pm 25 \% \mathrm{D} \text { (liq.), } \pm 30 \% \mathrm{D} \text { (solid) }\end{array}$ \\
\hline $\begin{array}{l}\text { Mercury by CVAA } \\
\text { (cold vapor atomic } \\
\text { absorption) }\end{array}$ & $\begin{array}{l}\text { SW846-7471A } \\
\text { SW846-7470 }\end{array}$ & $\begin{array}{l}\text { instrument blank } \\
\text { calibration verification (ICV \& CCV) } \\
\text { method blank (sample prep) } \\
\text { laboratory control sample (sample prep) } \\
\text { matrix spike } \\
\text { matrix spike duplicate or sample duplicate } \\
\text { post digestion spike }\end{array}$ & $\begin{array}{l}\text { none specified } \\
\text { none specified } \\
\text { none specified } \\
\text { none specified } \\
\text { none specified } \\
\text { none specified } \\
\text { none specified }\end{array}$ & $\begin{array}{c}<5 \times \text { IDL } \\
\pm 10 \% \mathrm{D} \\
<5 \times \mathrm{IDL} \\
\pm 25 \% \mathrm{D} \\
\pm 25 \% \mathrm{D} \text { (liq.), } \pm 30 \% \mathrm{D} \text { (solid) } \\
\pm 20 \mathrm{RPD} \text { (liq.), } \pm 30 \mathrm{RPD} \text { (solid) } \\
\pm 25 \% \mathrm{D} \text { (liq.), } \pm 30 \% \mathrm{D} \text { (solid) }\end{array}$ \\
\hline $\begin{array}{l}\text { Carbon (total } \\
\text { organic carbon, } \\
\text { total carbon, total } \\
\text { inorganic carbon) }\end{array}$ & SW846-9060 & $\begin{array}{l}\text { instrument blank } \\
\text { calibration verification (ICV \& CCV) } \\
\text { matrix spike } \\
\text { matrix spike duplicate }\end{array}$ & $\begin{array}{l}\text { none specified } \\
\text { none specified } \\
\text { none specified } \\
\text { none specified }\end{array}$ & $\begin{array}{c}<3 \times \text { IDL } \\
\pm 10 \% \mathrm{D} \text { (ICV.), } \pm 20 \% \mathrm{D} \text { (CCV) } \\
\pm 25 \% \mathrm{D} \text { (liq.), } \pm 30 \% \mathrm{D} \text { (solid) } \\
\pm 20 \text { RPD (liq.), } \pm 30 \text { RPD (solid) }\end{array}$ \\
\hline $\begin{array}{l}\text { Anions by lon } \\
\text { Chromatography } \\
\text { (IC) }\end{array}$ & SW846-9056 & $\begin{array}{l}\text { calibration verification (ICV \& CCV) } \\
\text { matrix spike } \\
\text { sample duplicate }\end{array}$ & $\begin{array}{c} \pm 10 \% \mathrm{D}(\mathrm{ICV}), \pm 5 \% \mathrm{D}(\mathrm{CCV}) \\
\text { none specified } \\
\text { none specified }\end{array}$ & $\begin{array}{c} \pm 10 \% \mathrm{D}(\mathrm{ICV}), \pm 15 \% \mathrm{D}(\mathrm{CCV}) \\
\pm 25 \% \mathrm{D} \\
\pm 20 \mathrm{RPD}\end{array}$ \\
\hline $\mathrm{pH}$ measurement & $\begin{array}{l}\text { SW846-9040A } \\
\text { SW846-9045B }\end{array}$ & $\begin{array}{l}\text { check standard } \\
\text { sample duplicate }\end{array}$ & $\begin{array}{l}\text { none specified } \\
\text { none specified }\end{array}$ & $\begin{array}{l} \pm 10 \% \mathrm{D} \\
\pm 20 \% \mathrm{D}\end{array}$ \\
\hline
\end{tabular}




\begin{tabular}{|c|c|c|c|c|}
\hline Analysis & $\begin{array}{l}\text { Method (s) } \\
\text { CASD-AM- }\end{array}$ & $\begin{array}{c}\text { Quality Control } \\
\text { Check } \\
\text { (per batch) }\end{array}$ & $\begin{array}{c}\text { SW }-846 \\
\text { Acceptance } \\
\text { Criteria } \\
\text { (\%D, \%R, RPD) } \\
\end{array}$ & $\begin{array}{c}\text { RMAL } \\
\text { Acceptance } \\
\text { Criteria } \\
(\% \mathrm{D}, \% \mathrm{R}, \mathrm{RPD}) \\
\end{array}$ \\
\hline $\begin{array}{l}\text { Total and dissolved } \\
\text { solids (TS \& TDS) }\end{array}$ & $\begin{array}{l}\text { EPA600-160.2 } \\
\text { EPA600-160.3 }\end{array}$ & $\begin{array}{l}\text { sample duplicate } \\
\text { check standard }\end{array}$ & $\begin{array}{l}\text { none specified } \\
\text { none specified }\end{array}$ & $\begin{array}{c} \pm 10 \mathrm{mg} / 10 \mathrm{~mL} \text { sample } \\
\pm 10 \% \mathrm{D}\end{array}$ \\
\hline $\begin{array}{l}\text { Carbonate and } \\
\text { bicarbonate titration }\end{array}$ & AC-MM-1 003105 & $\begin{array}{l}\text { sample duplicate } \\
\text { check standard }\end{array}$ & $\begin{array}{l}\text { none specified } \\
\text { none specified }\end{array}$ & $\begin{array}{l} \pm 20 \mathrm{RPD} \\
\pm 20 \% \mathrm{D}\end{array}$ \\
\hline Gross alpha/beta & $\begin{array}{l}\text { EPA-900.0 } \\
\text { RML-RA02 } \\
\text { RML-RA12 }\end{array}$ & $\begin{array}{l}\text { background check } \\
\text { calibration verification } \\
\text { method blank (optional) } \\
\text { sample duplicate } \\
\text { matrix spike }\end{array}$ & $\begin{array}{l}\text { none specified } \\
\text { none specified } \\
\text { none specified } \\
\text { none specified } \\
\text { none specified }\end{array}$ & $\begin{array}{c}<3 \text { sigma daily change } \\
\pm 10 \% \mathrm{D} \\
\text { evaluated for contamination } \\
\pm 25 \mathrm{RPD} \text { (liq.), } \pm 30 \mathrm{RPD} \text { (solid) } \\
\pm 25 \% \mathrm{D} \text { (liq.) } \& \pm 30 \% \mathrm{D} \text { (solid) }\end{array}$ \\
\hline $\begin{array}{l}\text { Vuclides by garmma } \\
\text { spectrometry }\end{array}$ & EPA-901.1 & $\begin{array}{l}\text { background check } \\
\text { calibration verification } \\
\text { sample duplicate }\end{array}$ & $\begin{array}{r}\text { none specified } \\
\text { none specified } \\
\text { none specified }\end{array}$ & $\begin{array}{c}\text { <3sigma daily change } \\
\pm 10 \% \mathrm{D} \\
\pm 25 \% \mathrm{D} \text { (liq.) } \& \pm 30 \% \mathrm{D} \text { (solid) }\end{array}$ \\
\hline 5̀r-90 determination & $\begin{array}{l}\text { RML-RA13 } \\
\text { EPA-905.0 }\end{array}$ & $\begin{array}{l}\text { method blank (optional) } \\
\text { laboratory control sample } \\
\text { matrix spike } \\
\text { matrix spike duplicate or sample duplicate } \\
\text { associated instrument QC }\end{array}$ & $\begin{array}{l}\text { none specified } \\
\text { none specified } \\
\text { none specified } \\
\text { none specified } \\
\text { none specified }\end{array}$ & $\begin{array}{l}\text { evaluated for contamination } \\
\qquad 20 \% \mathrm{D} \\
\pm 25 \% \mathrm{D} \text { (liq.)\& } 30 \% \mathrm{D} \text { (solid) } \\
\pm 25 \mathrm{RPD} \text { (liq.), } \pm 30 \mathrm{RPD} \text { (solid) } \\
\text { see gross alpha/beta criteria }\end{array}$ \\
\hline $\begin{array}{l}\text { 「c-99 } \\
\text { letermination }\end{array}$ & $\begin{array}{l}\text { DOE Compendium } \\
\text { RP550 } \\
\text { RML-RA05 }\end{array}$ & $\begin{array}{l}\text { method blank (optional) } \\
\text { laboratory control sample } \\
\text { matrix spike } \\
\text { matrix spike or sample duplicate } \\
\text { associated instrument } Q C\end{array}$ & $\begin{array}{l}\text { none specified } \\
\text { none specified } \\
\text { none specified } \\
\text { none specified } \\
\text { none specified }\end{array}$ & $\begin{array}{c}<3 \times \mathrm{IDL} \\
20 \% \mathrm{D} \\
\pm 25 \% \mathrm{D} \text { (liq.) } \& \pm 30 \% \mathrm{D} \text { (solid) } \\
-25 \mathrm{RPD} \text { (liq.) } \pm 30 \mathrm{RPD} \text { (solid) } \\
\text { see 1CP-MS criteria }\end{array}$ \\
\hline 4-3 determination & EPA-906.0 & $\begin{array}{l}\text { method blank (optional) } \\
\text { laboratory control sample } \\
\text { matrix spike } \\
\text { matrix spike duplicate or sample duplicate } \\
\text { associated instrument } Q C\end{array}$ & $\begin{array}{l}\text { none specified } \\
\text { none specified } \\
\text { none specified } \\
\text { none specified } \\
\text { none specified }\end{array}$ & $\begin{array}{l}\text { evaluated for contamination } \\
\qquad 20 \% \mathrm{D} \\
\pm 25 \% \mathrm{D} \text { (liq.) \& } \pm 30 \% \mathrm{D} \text { (solid) } \\
\pm 25 \mathrm{RPD} \text { (liq.), } \pm 30 \mathrm{RPD} \text { (solid) } \\
\text { see gross alpha/beta criteria }\end{array}$ \\
\hline Th Determination & $\begin{array}{l}\text { EPA-901.1 } \\
\text { RML-RA09 }\end{array}$ & $\begin{array}{l}\text { method blank (optional) } \\
\text { laboratory control sample } \\
\text { matrix spike } \\
\text { matrix spike duplicate or sample duplicate } \\
\text { associated instrument QC }\end{array}$ & $\begin{array}{l}\text { none specified } \\
\text { none specified } \\
\text { none specified } \\
\text { none specified } \\
\text { none specified }\end{array}$ & $\begin{array}{l}\text { evaluated for contamination } \\
\qquad 20 \% \mathrm{D} \\
\pm 25 \% \mathrm{D} \text { (liq.) \& } \pm 30 \% \mathrm{D} \text { (solid) } \\
-25 \mathrm{RPD} \text { (liq.), } \pm 30 \mathrm{RPD} \text { (solid) } \\
\text { see gamma spectrometry criteria }\end{array}$ \\
\hline $\begin{array}{l}\text { PCBs } \\
\text { (polychlorinated- } \\
\text { biphenyls) }\end{array}$ & SW846-8080 & $\begin{array}{l}\text { calibration verification (ICV \& CCV) } \\
\text { method blank (sample prep) } \\
\text { surrogate standard } \\
\text { matrix spike } \\
\text { matrix spike duplicate } \\
\text { sample duplicate } \\
\text { laboratory control sample (sample prep) }\end{array}$ & $\begin{array}{l}\text { refer to method } 8080 \\
\text { none specified } \\
\text { none specified } \\
\text { none specified } \\
\text { none specified } \\
\text { none specified } \\
\text { none specified } \\
\end{array}$ & $\begin{array}{l}\text { to be specified } \\
\text { < regulatory limit (2ppm) } \\
\quad \pm 50-150 \% R \\
\pm 50-150 \% R \\
\pm 50-150 \% R \\
\text { to be specified } \\
\text { to be specified }\end{array}$ \\
\hline
\end{tabular}

a Initial calibration verification (ICV) is typically performed at the beginning of a run to check the calibration and must be independent of the calibration standards. The continuing calibration verification (CCV) must also be independent of the calibration standards, but may be the same standard as the ICV. The CCV is typically analyzed every 10 samples and at the end of the run for metals analysis or every 12 samples for organic analysis.

b The calibration blank is an instrument blank used in the calibration to initially determine the blank value and therefore used as blank subtraction. The continuing calibration blank (CCB) is also an instrument blank which is analyzed every 10 samples and at the end of the run, but is not used in blank subtraction, but only to monitor instrument contamination. 
C Method blanks and laboratory control samples are only required if a sample preparation is performed before analysis. Sample preparation does not include dilutions or transfers to containers.

d Post digestion spikes are not necessary if the pre-digestion spike is in control. If this control does not meet the QC acceptance criteria, the post digestion spike should be performed.

e Acceptance criteria:

$\% \mathrm{D}=\%$ deviation from true value

$\% \mathbf{R}=\%$ recovery of true value

$\mathrm{RPD}=$ relative percent difference between two compared values

f Method blanks for radiochemical analysis are used to monitor cross contamination. However, due to the levels of radioactivity present in samples at the RMAL, the effect of contamination may be insignificant in most cases. Therefore, the requirement to analyze a method blank for radiochemical analysis is optional (i.e. at the discretion of the chemist or supervisor).

g Acceptance criteria for the method blanks performed for radiochemical analysis varies based upon the level of activity in the samples and the amount of background activity. A qualified chemist reviews the data from method blanks to determine if significant contamination is present.

h The acceptance criteria for PCB analyses which are not identified in this table, shall be specified at a later date. Currently, the Analytical Methods Group group leader specifies the QC criteria if different from SW846 and if not specified by the sample generator. 
B-4 


\section{INTERNAL DISTRIBUTION}

1-2. BJC PDCC at ETTP

3-4. Central Research Library

5. M. V. Buchanan

6-10. J. M. Giaquinto

11. R. D. George, BJC

12. K. P. Guay, BJC

13. R. T. Jubin

14-18. J. M. Keller

19. T. E. Kent

20-2 1. Laboratory Records Department - RC

22. D. D. Lee

23. B. D. Oakley, BJC

24. C. P. McGinnis

25-29 J. W. Moore, BJC

30. R. M. Robinson, BJC

31. R. D. Spence

32. C. B. Scott, BJC

33. P. A. Taylor

34. J. R. Trabalka

35. S. D. Van Hoesen

36. W. R. Zulliger, BJC 


\section{EXTERNAL DISTRIBUTION}

36-40. G. L. Riner

U. S. Department of Energy

P.O. Box 2001 MS-EM921

Oak Ridge, Tennessee 3783 1-8620

41-42. Bryan Roy

Foster Wheeler Environmental Corporation

111 Union Valley Road

Oak Ridge, Tennessee 37830-8697

43. Office of Scientific \& Technical Information

P. 0. Box 62

Oak Ridge, TN 37831 